University of Louisville

ThinkIR: The University of Louisville's Institutional Repository

\title{
Stretching adversely modulates locomotor capacity following spinal cord injury via activation of nociceptive afferents.
}

Anastasia V. Keller

Univeristy of Louisville

Follow this and additional works at: https://ir.library.louisville.edu/etd

Part of the Medicine and Health Sciences Commons

\section{Recommended Citation}

Keller, Anastasia V., "Stretching adversely modulates locomotor capacity following spinal cord injury via activation of nociceptive afferents." (2017). Electronic Theses and Dissertations. Paper 2791.

https://doi.org/10.18297/etd/2791

This Doctoral Dissertation is brought to you for free and open access by ThinkIR: The University of Louisville's Institutional Repository. It has been accepted for inclusion in Electronic Theses and Dissertations by an authorized administrator of ThinkIR: The University of Louisville's Institutional Repository. This title appears here courtesy of the author, who has retained all other copyrights. For more information, please contact thinkir@louisville.edu. 


\title{
STRETCHING ADVERSELY MODULATES LOCOMOTOR CAPACITY FOLLOWING SPINAL CORD INJURY VIA ACTIVATION OF NOCICEPTIVE AFFERENTS
}

\author{
By \\ Anastasia V. Keller \\ B.S., University of Louisville, 2010 \\ M.S., University of Louisville, 2012, 2014

\begin{abstract}
A Dissertation
Submitted to the Faculty of the School of Medicine at the University of Louisville in Partial Fulfillment of the Requirements for the Degree of
\end{abstract} \\ Doctor of Philosophy in Physiology and Biophysics \\ Department of Physiology and Biophysics \\ University of Louisville \\ Louisville, Kentucky
}

August 2017 
Copyright 2017 by Anastasia Keller

All rights reserved 



\title{
STRETCHING ADVERSELY MODULATES LOCOMOTOR CAPACITY FOLLOWING SPINAL CORD INJURY VIA ACTIVATION OF NOCICEPTIVE AFFERENTS
}

\author{
By \\ Anastasia Keller \\ B.S., University of Louisville, 2010 \\ M.S., University of Louisville, 2012, 2014
}

A Dissertation Approved on

June 9, 2017

by the following Dissertation Committee:

Dissertation Director: Dr. David S. K. Magnuson

Dr. Jeffrey Petruska

Dr. Dena Howland

Dr. James Hoying

Dr. David Lominadze

Dr. Dale Schuschke 


\section{DEDICATION}

In the loving memory of my father, Valery Prokopenko (July 31, 1941 - June 2, 2010) whom I admired for his sacrificial love for our family, wisdom, dedication to excellence in all he set out to accomplish and innovative thinking in his work. I would not be here without his influence and belief in me. 


\section{ACKNOWLEDGMENTS}

First, I would like to thank Dr. Magnuson for his excellent mentorship and leadership. I felt very confident and secure while navigating through the graduate school because of his steadfast support and open-door policy at all times. I truly enjoyed all of the insightful conversations that we have had pertaining not only to science. I could not have asked for a better mentor. Next, I would like to thank Dr. Petruska for providing me with direction at the very crucial and pivotal point in my $\mathrm{PhD}$ work. His ideas were instrumental for the last experiment. I thank all of my committee members: Dr. Lominadze, Dr. Howland, Dr. Hoying, Dr. Schuschke for helping me to refine my thoughts and ideas, the overall input and support as I journeyed through this process. I would like to extend a special and warm thank you to Alice Shum-Siu for taking me under her wing as I joined the lab. From day one she has taught me everything from how to handle animals to the magic of immunohistochemistry. I would be so lost without her guidance, friendship and loving support. This work would not be possible without the great team of lab technicians and bioengineering co-ops who have helped with the stretching sessions and data analysis, I thank them all from the bottom of my heart, this $\mathrm{PhD}$ thesis is stapled by the hours of their hard work. Likewise, the Kentucky Spinal Cord Injury core, specifically Christine Yarberry, Johnny Morehouse, Darlene Burke and Jason Beare have been so incredibly helpful. I could not have done all the surgeries, data collection and analysis without them. Thank you to Dr. Powel and Dr. Drake for helping me address any veterinary concerns that have come up during the studies. Because of their oversight and guidance regarding animal 
care most of my subjects were healthy and made it to the end of the study. Thank you to RRC stuff for keeping our animal housing conditions excellent and for providing special accommodation when needed for the experimental purposes and health of the animals. Thank you to my fellow graduate students: Amanda Pocratsky, Katie Harman, Katie DeVeau, Courtney Shephard and Greg States. They were always there for me with the one of a kind perspective. This experience would not be as fun without them. Thank you to all the members of the Kentucky Spinal Cord Injury Center. I thank Dr. Terson de Paleville and Dr. Swank for encouraging me to pursue my PhD. I thank my family and friends for their unwavering support, for listening and encouraging me through all the fun and hard times. I would not be where I am today without them. I thank my God for guiding me, inspiring me and giving me strength during some of the most difficult times of my life in the past five years. 


\section{ABSTRACT \\ STRETCHING ADVERSELY MODULATES LOCOMOTOR CAPACITY FOLLOWING SPINAL CORD INJURY VIA ACTIVATION OF NOCICEPTIVE AFFERENTS

\author{
Anastasia V. Keller
} \\ June 9, 2017}

Spinal cord injury (SCI) is the second leading cause of paralysis in the United States, affecting around 282,000 people with 17,000 new cases each year. Initial and secondary damage to the spinal cord disrupts multiple descending pathways that modulate the function of sympathetic preganglionic neurons and central pattern generating circuitry. Resulting loss of autonomic and locomotor functions, as well as decreased levels of physical activity, lead to a myriad of complications that affect multiple organ systems and significantly reduce both quality of life and life expectancy in individuals with SCI. Spasticity and muscle contractures are two common secondary conditions that develop in the chronic stages of SCI as a result of neurobiological and soft tissue adaptations. Stretching is the widely accepted initial therapy for the treatment of both spasticity and muscle contractures.

Unlike humans, rats with experimental incomplete SCI have robust locomotor recovery and do not develop significant muscle contractures or spasticity. One of the longstanding operating principles in the Magnuson laboratory is that rats retrain or rehabilitate themselves through large amounts of in-cage activity. A previous graduate student in our 
lab, Krista Caudle, tested this hypothesis using custom designed wheelchairs to immobilize Sprague Dawley rats with mild-moderate SCIs. As expected, the immobilized SCI animals did not recover their locomotor function and, in addition, developed muscle contractures. To mimic the approach used in the clinic for the treatment of contractures, a hindlimb stretching protocol was developed and implemented as part of our daily care routine. As a control, non-immobilized SCI rats also received stretching therapy. Surprisingly, stretched rats and wheelchair immobilized rats showed similar impairments in locomotor recovery. This finding was alarming and warranted further studies.

The work presented in this thesis is a continuation of the stretching projects in the Magnuson laboratory. Four major studies were carried out in order to improve our understanding of this stretching phenomenon and to begin uncovering the underlying physiological mechanisms. The following experiments revealed that hindlimb stretching disrupts locomotor function in rats with acute and chronic moderately-severe SCI. We also determined that dynamic "range of motion" stretching resulted in a similar pattern of locomotor impairment as our standard static stretch-and-hold protocol in rats with moderate sub-acute SCIs. Furthermore, using kinematics and electromyography (EMG), we determined that one of the most frequent responses to stretch in the rat hindlimbs is similar to human clonus. The significance of these findings are three-fold. First, to our knowledge, there has not been a specific description of clonus in the rat model of the SCI previously. Second, the similarity of the responses to stretch between rats and humans make a compelling argument for the clinical relevance of the stretching phenomenon. Finally, we determined that stretch-induced locomotor deficits depend on the presence of nociceptive afferents. Speculations about the specific physiological mechanisms of the 
stretching phenomenon and future directions are discussed. Comprehensive review of the stretching literature revealed a major problem in the rationale that is frequently provided for the use of stretching in the management of muscle contractures after SCI. In light of this work, a perspective on the future of stretching therapy in the rehabilitation after SCI is provided. 
TABLE OF CONTENTS

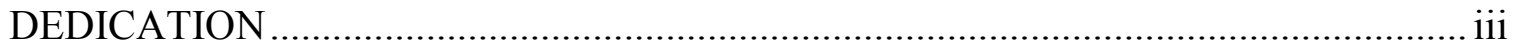

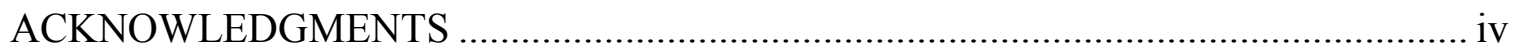

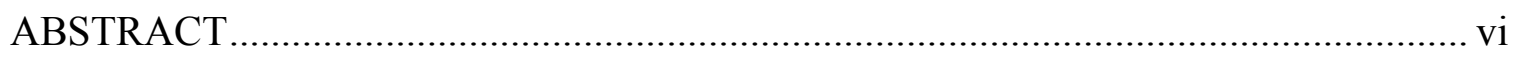

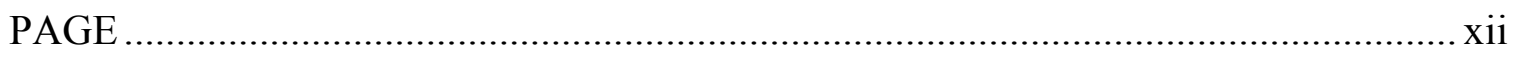

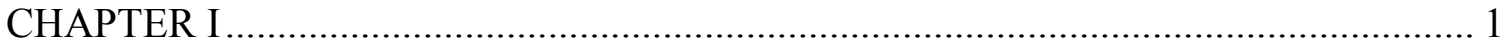

EPIDEMIOLOGY, PATHOPHYSIOLOGY AND SECONDARY COMPLICATIONS

FOLLOWING SPINAL CORD INJURY. ..................................................................

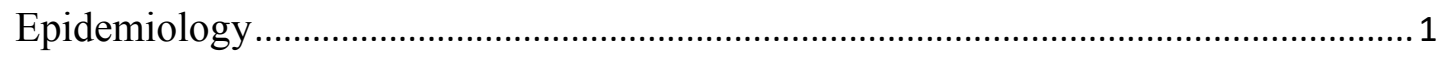

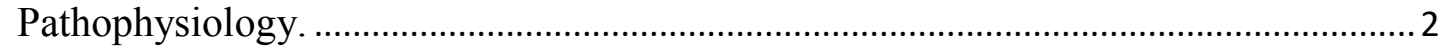

Secondary Complications: Spasticity .......................................................................

Secondary Complications: Muscle Contractures. .............................................................

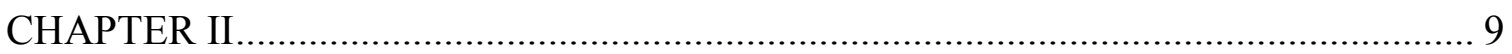

REHABILITATION FOLLOWING SPINAL CORD INJURY ……………………....9

Stretching Therapy for Treatment of Muscle Contractures and Spasticity ....................

Rehabilitation of Locomotor Function after Spinal Cord Injury ..................................17

Effects of Hindlimb Muscle Stretching on Locomotor Function in SCI rats..............28

Specific Aims and Hypotheses ................................................................................

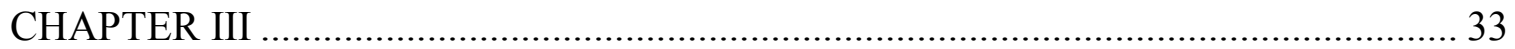

DISRUPTION OF LOCOMOTION IN RESPONSE TO HINDLIMB MUSCLE STRETCH AT ACUTE AND CHRONIC TIME POINTS AFTER A SPINAL CORD

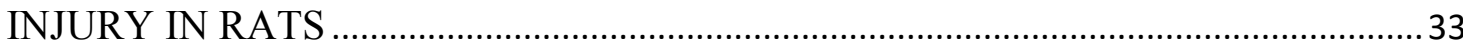

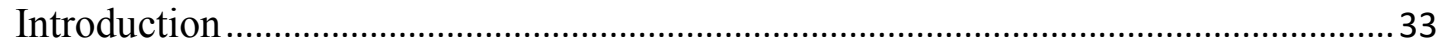

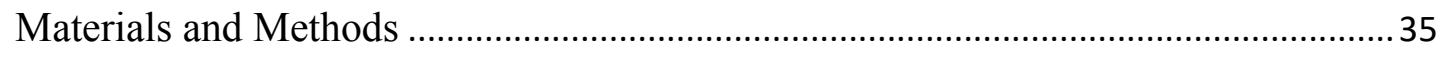

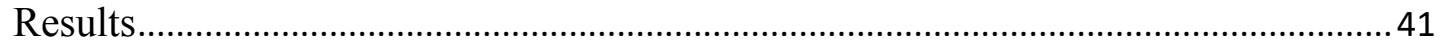

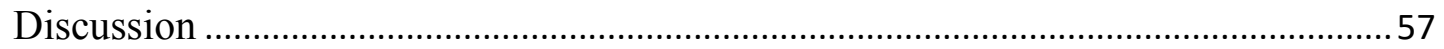

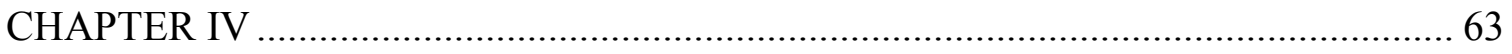


DYNAMIC “RANGE OF MOTION" HINDLIMB STRETCHING DISRUPTS LOCOMOTOR FUNCTION IN RATS WITH MODERATE SUBACUTE SPINAL CORD INJURIES

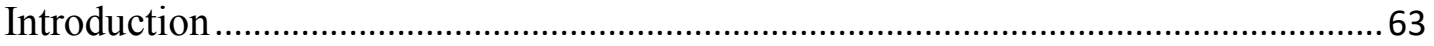

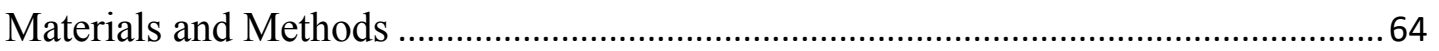

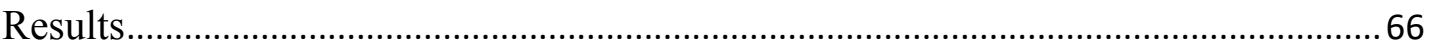

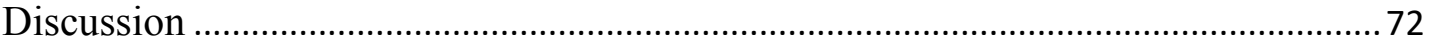

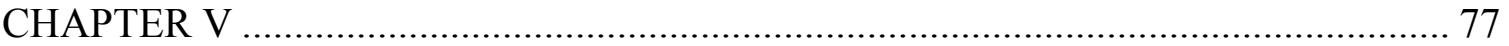

ELECTROMYOGRAPHIC PATTERNS OF THE RAT HINDLIMB IN RESPONSE

TO MUSCLE STRETCH AFTER SPINAL CORD INJURY .......................................77

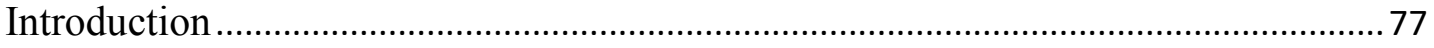

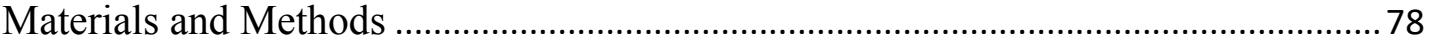

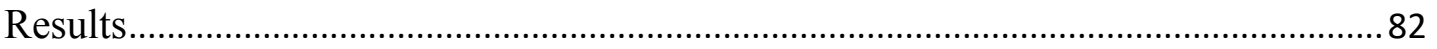

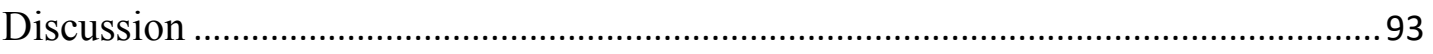

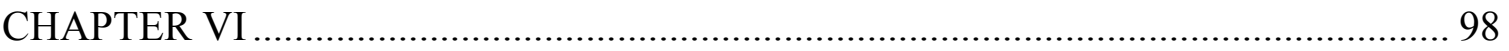

ROLE OF NOCICEPTIVE AFFERENTS IN STRETCH-INDUCED LOCOMOTOR DEFICITS IN RATS WITH SPINAL CORD INJURIES ............................................98

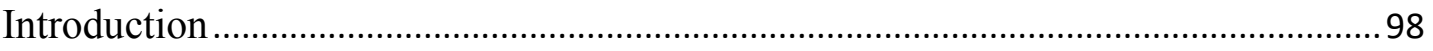

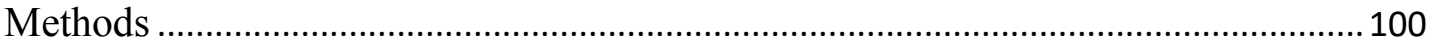

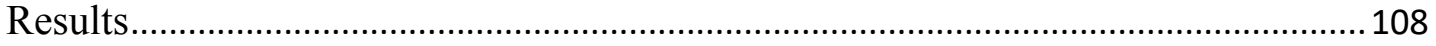

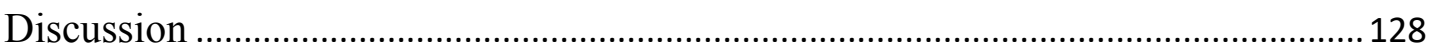

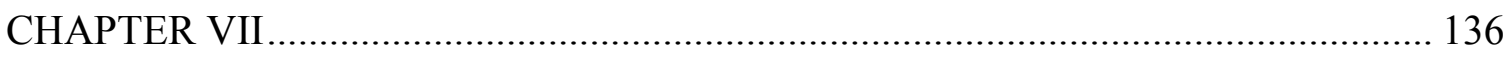

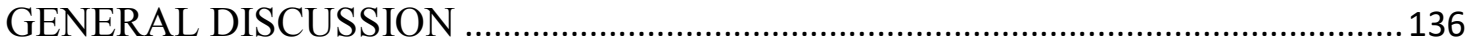

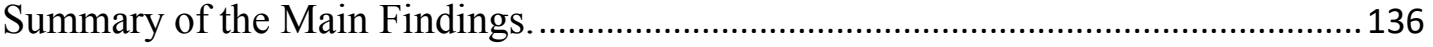

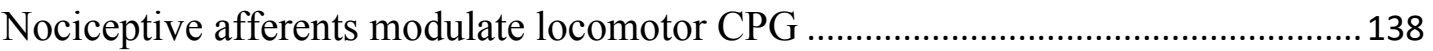

Possible mechanisms of nociceptive afferent dependent stretch-induced locomotor

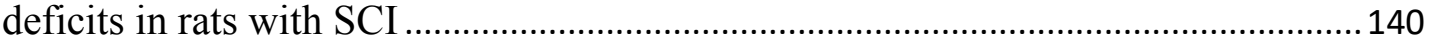

Future directions 1: deciphering physiological mechanisms....................................143

Consideration of the physiological role of group III and IV afferents after SCI......145

Future directions 2: stretching and cardiovascular function ...................................... 147

Rationale for stretching: methodological considerations .......................................... 148 
How stretching brings about rapid increase in ROM in healthy humans and why it isn't effective after SCI........................................................................................... 150

Mechanism of stretch-induced strength loss and its relationship to spasticity .........153

Future of stretching therapy in rehabilitation after SCI ...........................................154

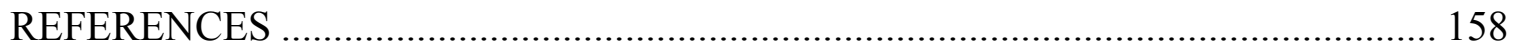

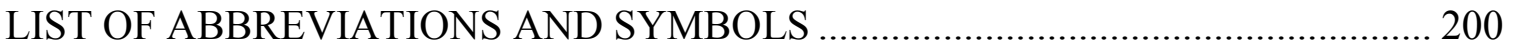

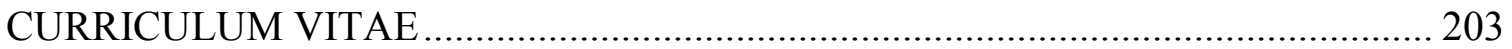




\section{LIST OF FIGURES}

PAGE

Figure 1. Time-line indicating the primary events of the acute and chronic stretch study including the outcome measures and weeks of stretching relative to the time of injury.. 37 Figure 2. Locomotor function of the animals with sub-acute SCI in response to dynamic stretching protocol. 44

Figure 3. Kinematic and gait analysis of shallow water walking (SWW) of the animals stretched at acute and chronic time points after SCI.

Figure 4. Nocturnal in-cage activity of the animals stretched at acute and chronic time points after SCI.

Figure 5. Amplitude of magnetically evoked muscle responses (MEMRs) of the animals stretched at acute and chronic time points after SCI. 52 Figure 6. Passive end range of motion of the animals stretched at acute and chronic time points after SCI.

Figure 7. Locomotor function of the animals with sub-acute SCI in response to dynamic stretching protocol. 70

Figure 8. Characteristics of the vibration response to the dynamic stretching protocol in rats with sub-acute SCI.

Figure 9. Three commonly occurring EMG responses during hindlimb stretching knee muscles of the rats with SCI.

Figure 10. Examples of EMG responses during a 10 second positioning period prior to quadriceps stretch at 2 and 8 weeks post SCI.

Figure 11. EMG and force traces during a 1 minute quadriceps stretch

Figure 12. Quantification of amplitude and frequency of clonic-like EMG responses to stretch in the contralateral limb at week 2 and 8 , and ipsilateral limbs at week 8 . 
Figure 14. BBB Open Field Locomotor Scores, 3D kinematics and gait analysis of the animals after SCI and during stretching.

Figure 15. Baseline sensory function of capsaicin or vehicle-treated and control (untreated) animals

Figure 16. Baseline locomotor function of capsaicin or vehicle-treated and control (untreated) animals.

Figure 17. Characteristics of magnetically-evoked muscle potentials of capsaicin or vehicle-treated and control animals at baseline and after SCI.

Figure 18. Locomotor function of capsaicin or vehicle-treated and control animals after SCI and during stretching.

Figure 19. Images and quantification of CGRP immunoreactivity in the dorsal horn of the capsaicin or vehicle-treated and control animals.

Figure 20. Quantification of the total number of c-Fos + neurons throughout the lumbar enlargement of the capsaicin or vehicle treated and control animals and the schematic representation of their distribution. The bars represent an average number of c-Fos + neurons from 3 sections at each level, while each dot represents individual animals (A). $\mathrm{n}=8$ per group for each level except at L1 and L5 CAP: $\mathrm{n}=6$ and at L1 VEH \& CON n=7, each. Analyzed using RM ANOVA, Bonferroni HSD post hoc, $\mathrm{p}<.05$. Traces of spinal cord sections representative of each group for each level with black dots corresponds to c-Fos + neurons within the chosen sections (B). 120

Figure 21. Images of spinal cord sections stained for c-Fos. 121 Figure 22. Number of c-Fos + neurons separated into three general regions of gray matter.

Figure 23. Quantification of the four major hindlimb responses during stretching and scatterplots of the kicking and vibration responses vs. the number of c-Fos+ neurons.. 125 Figure 24. Hindlimb muscle analysis for presence of centralized nuclei and muscle fiber cross sectional area. 127 Figure 25. Summary of the main findings. 


\section{CHAPTER I}

\section{EPIDEMIOLOGY, PATHOPHYSIOLOGY AND SECONDARY COMPLICATIONS FOLLOWING SPINAL CORD INJURY}

\section{Epidemiology}

According to the National Spinal Cord Injury Statistical Center (NSCISC) there are currently approximately 282,000 persons who live with a spinal cord injury in the US. Seventeen thousand new spinal cord injury (SCI) cases occur each year with motor vehicle accidents still remaining a leading etiology for this condition affecting predominantly young males, although the age at the time of injury has increased from average of 29 years to 42 years over the past 40 years. The majority of injuries happen in the cervical region of the spinal cord resulting in tetraplegia and thus have a myriad of serious consequences that not only affect sensory and motor functions, but also result in impairments in the autonomic nervous system that normally regulate cardiovascular, gastrointestinal, urinary and other organ systems. People with SCI are at an increased risk of developing heart, metabolic and respiratory diseases and overall have a significantly reduced life expectancy compared to the uninjured general population. Severe spinal cord injury undoubtedly is a devastating life altering event that in addition to person's physiological and psychological detriments also imposes a significant financial burden, as millions of dollars are spent on consequent lifelong healthcare and living expenses [1]. The underlying cause of such poor outcomes and dreadful prognosis in the majority of SCI cases is that central nervous system (CNS) 
does not regenerate [2]. Numerous research studies have been conducted over the past 70 years focusing on repair of the damaged neuronal connections across the spinal cord lesion involving different techniques from growth factor delivery $[3,4]$ to transplantations of various kinds of cells [5-9] into the SCI epicenter. Unfortunately, none of the studies resulted in any significant functional improvement and despite superior efforts the evidence of meaningful anatomical/structural changes (neuronal regeneration) at the lesion site following such manipulations is lacking with the exception of few studies [10-12]. Nevertheless, the research in this area is ongoing with the intense focus on cell therapy and more specifically combination of cell therapies, as it is becoming evident that a single origin cell transplantation would likely have no effect [13]. It is also apparent that the view of the potential of cell therapies in the treatment of SCI is shifting from rather being able to induce a regenerative response to playing a role in neuroprotection of the nervous tissue spared on the impact from the secondary damage [13].

\section{Pathophysiology.}

In traumatic SCI the initial mechanical insult to the spinal cord primarily disrupts central gray matter due to its softer consistency and higher vascularity [14]. Over the following hours a cascade of interrelated events such as oxidative stress, infiltration of inflammatory cells and massive release of certain neurotransmitters at the epicenter initiates the secondary phase of spinal cord damage. The resulting local inflammatory environment makes the survival of any remaining neurons and oligodendrocytes extremely challenging and many cells spared from the initial mechanical damage undergo apoptotic cell death. $[15,16]$.

The understanding of pathophysiological processes that occur at the onset of SCI mostly comes from the various animal models of injury. First, mechanical disruption of the 
microvasculature in the spinal cord leads to hemorrhage and thrombosis that in turn results in ischemia and hypoperfusion in surrounding tissues [17]. During the ischemic period the tissues are forced to produce their energy through anaerobic metabolism, byproducts of which (eg. lactate) lower the perivascular $\mathrm{pH}$ causing reactive hyperemia. Reperfusion brings about oxygen-derived free radicals that rapidly react with cellular membranes (lipid peroxidation) initiating a vicious cycle of oxidation generated free radicals that furthers the cell damage $[16,18,19]$.

Another well recognized contributor to the secondary phase of SCI is excessive extracellular presence of glutamate, a predominant excitatory neurotransmitter in the CNS. Spinal cord injury results in massive membrane depolarization and subsequent glutamate release [20]. Glutamate binds to its receptors N-methyl-D-aspartate (NMDA) and alphaamino-3-hydroxy-5-methyl-isoxazolepropionate (AMPA)/kainite receptors) on the cell surfaces causing calcium and sodium influx. Overstimulation of these receptors results in excitotoxic injury to the cell due to accumulation of intracellular calcium [21-23]. Increases in calcium ions can trigger additional release of calcium from intracellular stores further exacerbating already disturbed cellular homeostasis. Calcium concentration is normally under tight regulation due to its important role in multiple intracellular signaling pathways. Its rapid accumulation inside the cell as a result of NMDA receptor overstimulation leads to activation of several calcium-dependent proteases and lipases such as calpains and phospholipase A2 that readily degrade crucial cellular components: membranes, neurofilaments and structural proteins of the axon-myelin unit $[24,25]$. Furthermore, calcium interferes with the mitochondrial ability to produce adenosine triphosphate (ATP) [26]. Increased concentration of sodium reduces the activity of sodium-potassium ATPase 
as well as sodium-calcium exchanger and thus also contributes to homeostatic dysregulation. These rapid changes in ionic distributions across cellular membrane and their effects within the cell ultimately result in initiation of apoptosis.

Disruption of homeostasis of the spinal cord tissue after its injury is characterized by the presence of damage-associated molecular patterns (DAMPs) which serve as major attractants to the immune cells resident in the CNS (microglia) as well as blood-borne immune cells (neutrophils, monocytes, leukocytes etc)[27]. DAMPs include intracellular proteins such as interleukin (IL)-1 $\alpha$ and IL-33, nucleic acids (particularly mitochondrial deoxyribonucleic acid (DNA)) and their derivatives (ATP, uric acid) all of which are normally present in high concentration only inside the cell [28-30]. Within a particular time course from seconds to days post injury, different types of immune cells migrate into the injury epicenter and initiate their responses which have been shown to have both detrimental and beneficial effects $[31,32]$. First responders, resident microglia exhibit rapid chemotaxis towards increased ATP concentration gradients [32]. ATP signaling facilitates the assembly of inflammasomes, a multiprotein complex responsible for production of proinflammatory cytokines IL-1 $\beta$ and IL-18 [33, 34]. ATP-induced inflammasome production also appears to influence neutrophil recruitment into the injury site, where they secrete pathogen-fighting compounds, such as proteases, inflammatory cytokines and free radicals [35]. In cases of sterile spinal cord injury (without an open wound), these molecules have no protective benefits, but rather exacerbate the toxic environment and thus contribute to secondary tissue damage. This concept finds support in studies that sought to inhibit neutrophil migration into damaged CNS and after successfully doing so, found increased tissue sparing and improved functional recovery $[36,37]$. Much 
debate exists about the nature of monocyte-derived macrophage effects at the SCI epicenter. On one hand, infiltration and activation by tumor necrosis factor alpha (TNF- $\alpha$ ), a cytokine released by many immune cells in response to SCI, they secrete glutamate that is already present in toxic amounts at the injury site [38]. In addition, macrophages producing nitric oxide synthase contribute to the generation of free radicals and thus play a role in secondary cell death [31]. On the other hand, a multitude of studies have shown that macrophages have an important role in tissue protection through secretion of neurotrophic factors and regeneration after SCI as they also release the atypical growth factor oncomodulin $[39,40]$. Moreover, their phagocytic function is crucial for removal of the myelin debris that inhibits regeneration. The studies that focused on promoting macrophage activity provided convincing evidence that overall these cells have a beneficial effect on the outcomes after CNS injury, whereas inhibition of their function was associated with poorer outcomes $[41,42]$. Even though the field of inflammation after CNS injury has overall considered the immune system to play a detrimental role in secondary cell death and has focused on developing therapies that attenuate the immune response, more and more evidence has come out to suggest that the immune system is important in the protection of spared neurons and initiation of healing and repair [43].

\section{Secondary Complications: Spasticity.}

Beyond the pathophysiological mechanisms responsible for initial and secondary cell death at the SCI epicenter, the regions of the spinal cord important for generation of locomotion in most cases not directly affected by the traumatic injury [1], also go through changes at chronic stages of SCI [44].

Among secondary complications following traumatic upper motor neuron lesion, are spasticity and muscle contractures $[45,46]$. Both of these conditions affect the 
musculoskeletal system and can significantly disrupt activities of daily living, such as dressing and transfers from bed to a wheelchair [47-50]. A common definition of spasticity stems from its clinical presentation as a velocity dependent increase in resistance to muscle stretch[51]. Hypereflexia is another term for spasticity due to the observation of increased excitability of the Ia afferent reflex pathway, likely due to impairments in both the presynaptic inhibition and homosynaptic depression of these afferents [52, 53]. Meanwhile, several inhibitory reflexes (disynaptic reciprocal Ia inhibition and autogenic Ib inhibition) appear diminished in spastic patients [52]. Fundamentally, spasticity reflects a loss of the normally highly-controlled and orderly recruitment of motoneuronal pools as well as modulation and coordination of their activity [54]. This is easily seen when a patient with spasticity attempts a functional movement (ex. pick up a glass of water) but a forceful agonist-antagonist co-contraction of the arm muscles renders that action unsuccessful. Another characteristic of spasticity is an altered ability for sensory input processing at the central level. Therefore, cutaneous, muscle or joint afferent stimuli often result in muscle spasms [55]. Instances of spontaneous muscle spasms, when there is no identifiable stimulus for their initiation, also occur in patients with spasticity [54].

Several neurobiological changes take place at the level of motor circuitry deprived of normal supraspinal input that have been proposed to underlie the development of spasticity. One of the key intrinsic properties of motoneurons is the generation of persistent inward currents (PICs) through low threshold calcium and sodium voltage gated ion channels in response to brief synaptic input [56]. Physiologically, these currents amplify and prolong neuronal activation, enabling sustained muscle contractions that, for example, are necessary for postural control [57]. Serotonergic (5-hydroxytryptamine (5-HT) and 
noradrenergic pathways that originate supraspinally modulate the state of motoneuronal excitability primarily through facilitation of PICs $[58,59]$. Remarkable flaccid paralysis acutely after a SCI is partly due to the disruption of the descending neuromodulatory inputs to the motor circuitry below the level of lesion [60]. Overtime, motoneurons compensate for the loss of descending serotonergic inputs via the upregulation of a constitutively active isoform of the 5-HT receptor $\left(5-\mathrm{HT}_{2} \mathrm{C}\right)$ and thus restore their capacity to generate large PICs even though serotonin levels remain significantly diminished [61]. Normally, the activity of motorneurons is regulated by local inhibitory circuits that utilize gammaAminobutyric acid (GABA) and glycine neurotransmitters that act on chloride channels [62]. Entry of chloride ions into the cell down the electrochemical gradient which is established by potassium-chloride exchanger ( $\mathrm{KCC} 2$ ) hyperpolarizes the cell and stops PICs. SCI results in the downregulation of $\mathrm{KCC} 2$ channels [63]. The resulting decrease in chloride equilibrium potential reverses the function of normally inhibitory neurotransmitters into an excitatory phenotype. Therefore, the compensatory changes of increased excitability and dysregulation of chloride ion homeostasis essential for inhibitory modulation of motoneuron activity results in poorly controllable motoneuron discharges manifested as muscle spasms.

\section{Secondary Complications: Muscle Contractures.}

Skeletal muscles are the effector organs of motoneurons and their contractile property is the key characteristic for their function that underlies human movement. Skeletal muscle tissue possesses a remarkable ability to adapt and is capable of significant hypertrophy under high demands, such as strength training or can undergo substantial atrophy within just few weeks of immobilization[64]. Severe SCI results in muscle paralysis (at least initially) and is often followed by prolonged periods of immobility [65]. These conditions 
predispose patients with SCI to muscle wasting and development of related pathologies, such as joint contractures. Characteristic decrease in the passive range of motion as a result of joint contracture limits function and the independence of a person with SCI [48, 49]. In some cases, contractures result in disability that would normally be associated with a higher level lesion [47].

In addition joint contractures can be a source of pain and lead to the development of pressure sores $[45,66]$. Muscle contractures begin slowly with changes in soft tissue. Muscle atrophy (decrease in muscle fiber size) occurs due to a reduction in protein synthesis and a switch to a catabolic state as a result of unloading [67]. In cases of immobilization of a muscle in shortened position, there is also the loss of serial sarcomeres [68]. With continual disuse the amount of connective tissue within the muscle increases [69]. These initial changes have a clinical presentation of reduced muscle compliance in response to passive stretch. Significant changes also occur within the joint structures. Fibro-fatty connective tissue proliferates within the joint space while cartilage degenerates [70]. Formation of adhesions between the fibro-fatty connective tissue with cartilage and between the synovial folds as well as disorganization of cellular and fibrillary components within ligaments occur as a result of joint immobility [71, 72]. Development of spasticity also contributes to the pathophysiology of contractures by furthering the loss of sarcomeres and increasing connective tissue within the spastic muscle [66]. Unmanaged contractures can significantly deform joints rendering them dysfunctional [45]. 
CHAPTER II

REHABILITATION FOLLOWING SPINAL CORD INJURY

\section{Stretching Therapy for Treatment of Muscle Contractures and Spasticity}

Stretching remains a leading therapy for the treatment of muscle contractures and spasticity [73]. The rationale for the inclusion of stretching in the rehabilitation of these conditions most likely comes from the literature on flexibility training in the non-injured human population as well as basic animal science studies on muscle tissue responses to immobility and stretching.

Flexibility has been recognized as an important component of physical fitness [74]. Several benefits commonly attributed to flexibility training include injury prevention, reduction of muscle soreness, skill enhancement and muscle relaxation [75]. On the other hand, lack or limited flexibility is associated with increased risk of musculoskeletal injuries. The goal of stretching as a fundamental component of flexibility training is to increase range of motion (ROM) around the joints. Therefore, the effectiveness of stretching intervention is assessed by measuring joint ROM pre- and post- stretch using goniometer. Depending on the study, researchers ask human subjects to stretch until the sensation of resistance, tightness, discomfort or pain to ensure that the limb is as close to the end ROM as possible before taking the angle measurements.

Different types of stretching techniques exist: static, dynamic and several forms of proprioceptive neuromuscular facilitation (PNF) protocols and each has been shown to increase ROM [76]. Static stretching involves positioning muscle at its end ROM where 
it is held passively for some amount of time. Dynamic or cyclic stretching is performed by repeatedly moving the limb through its ROM with either no or short hold at the end ROM [77]. Several PNF stretching techniques utilize pre-stretch muscle contraction of either the muscle to undergo the stretch or the antagonistic muscle following relaxation and application of stretch. Several studies comparing different stretching methods concluded that PNF stretching brings about greater increase in ROM, although one study directly comparing static and PNF techniques have found static stretching to be more affective. Studies using static stretching of hamstring muscles are the most abundant perhaps due to the increased susceptibility of this muscle group to sustain an injury.

The effects of stretch on ROM in healthy human subjects can be seen immediately after stretch. Magnusson et al., found a significant increase in ROM after a single 90 second static hamstring stretch [78]. Nishikawa et al., found that just 3 by 10 seconds of either passive (limb extended by an examiner) or active (self-administered) stretches increased ROM by 15.8 or 7 degrees, respectively [79]. In another study 4 by 5 second passive stretches resulted in a 4.5 degree increase in ROM acutely [80]. Most studies however have examined stretching paradigms much longer in duration in order to provide guidelines for the development of effective flexibility training programs [76, 81-84]. Studies by Bandy et al., have provided a golden standard for duration and frequency of static stretching. In one study the researchers compared three groups of subjects that used different stretch durations: 15, 30 and 60 seconds in a stretching protocol of 5 days a week for 6 weeks. From the results they concluded that 30 seconds is an optimal stretch duration, since the subjects in that group had greater improvements in ROM than those in 15 second group, while the stretch duration of 60 seconds did not result in additional improvement in ROM. 
In a follow-up study, the same authors investigated the effects of increasing the frequency of stretching from 1 to 3 times a day. They still utilized two different stretch durations of 30 and 60 seconds with the same stretching protocol of 5 days a week for 6 weeks. Once again they did not find a significant difference between the two stretch durations and furthermore, no differences were found between the two frequency groups ( 1 vs 3 times a day). A more recent study by different authors examined a single 30 vs 60 seconds hamstring stretch also did not find any differences in ROM improvement between the two groups [85]. Convincing evidence exists to conclude that a stretching protocol consisting of 30 second static stretches either acutely or as part of a flexibility training program (3 times a week for 6 weeks) is sufficient to improve flexibility in the general healthy population. A recent systematic review with meta-analysis also confirmed that static stretching is effective at increasing hamstring flexibility in young healthy adults [86].

Ample evidence of the effectiveness of stretching for improving ROM in the healthy human population serves as a good basis for examining the therapeutic potential of stretching for more serious pathologic conditions affecting joint ROM, such as muscle contractures. Evidence from basic animal studies suggests that stretching can prevent immobility-induced pathologic changes in morphology of the muscles and connective tissue $[68,87,88]$. Specifically, Williams and colleagues used intermittent stretch on ankle muscles that were immobilized for 10 days in mice and found that 15 minutes of static stretch every other day was sufficient to prevent some loss in ROM compared to the immobilized non-stretched group. Morphological assessment of the soleus muscle showed that animals who received stretching had significantly lower connective tissue content within the muscle, although the stretching protocol used in this study was not effective at 
preventing the loss of sarcomeres in series [87]. In a follow up study, this group showed that daily stretching of 30 minutes during the two week immobilization period was not only sufficient to prevent the loss of sarcomeres, but actually resulted in an increase in the number of serial sarcomeres as compared to non-immobilized control muscles. As a result, the range of motion was maintained at or near a normal level throughout the immobilization weeks. Furthermore, stretching significantly reduced muscle wasting associated with immobility: stretched animals had only $9 \%$ loss of muscle weight as compared to $48 \%$ loss in non-stretched immobilized mice [68]. These and other animal studies [89, 90] have given much promise for the therapeutic use of stretching in rehabilitation of contractures for patients with SCI.

The efficacy of practically every stretching protocol for improvement in flexibility in healthy subjects, in addition to evidence that stretching leads to adaptive remodeling of the tissues from animal studies have made stretching the gold standard for rehabilitation of conditions affecting joint ROM (contractures and spasticity) for patients with SCI. Whereas studies in healthy subjects have led to some general guidelines about the effective duration and frequency of stretching to increase flexibility (at least acutely), no clear parameters or dosage of stretching in rehabilitation of contractures has been established probably due to mixed results reported by different researchers. A few studies have demonstrated that prolonged stretching administered using various techniques such as serial casting, soft and progressive splinting is efficacious for improving ROM in joint contractures. Specifically, a study by Booth et al., found that serial casting of ankles in patients with cortical lesions was effective at increasing joint ROM. The mean duration of their treatment was 22-39 days for different sub-groups [91]. In the study of children and 
young adult patients with cerebral palsy affected by severe knee flexion contracture, overnight soft splinting for 10 months resulted in a significant reduction in contractures [92]. In another study, elbow flexion contractures associated with bone fractures in nonneurological patients that were refractory to traditional static splitting and physiotherapy were improved by using orthosis with a turnbuckle that allowed for progressive stretching as tissue adapted to the new lengths. [93]. On the other hand, Harvey et al., examined the effects of 30 minutes of passive stretch applied every weekday for 4 weeks on ankle mobility in patients with SCI and found no change in the main outcome measures (passive resistance to stretch) [94]. A similar protocol was used to study patients with SCI except the stretching was applied to hamstring muscles. No improvements in hamstring extensibility were detected [95]. A longer duration of stretching (12 hours) applied to thumbs affected by contracture in patients with SCI in a study conducted by different authors did not lead to resolution of the contracture. The authors speculated that the splints used for this intervention did not provide a large enough torque [96]. One animal study showed that both duration and torque are important factors in successful contracture management (focused on prevention). The authors investigated 6 different protocols: long duration $(60 \mathrm{~min})$, short duration $(30 \mathrm{~min})$ or intermittent $(60 \mathrm{~second}$ stretch/30 second release for $30 \mathrm{~min}$ ) and each duration was examined under high or low torque conditions in a clinically relevant knee contracture model following SCI in rats (complete transection at T8). The intervention began one day following spinal cord transection. The authors found that the high torque long duration stretch was the most effective at significantly reducing knee contracture, however, it still did not completely prevent the decrease in ROM resulting from SCI [72]. 
Although stretching has been widely used for management of contractures, research on this topic does not show overwhelming support for its effectiveness. On the contrary, two systematic reviews on this topic have concluded that stretching does not lead to clinically meaningful changes in joint mobility $[97,98]$. Harvey et al., who was the first to conduct a randomized controlled study examining the effects of stretch on ankle mobility in patients with SCI, regardless of negative findings, still recommends continual use of stretching until more high quality studies fill in the gaps of our understanding on this issue. The author advises that perhaps stretching as it is currently administered in clinical settings (2-5 minutes for each major muscle group) is not sufficient and recommends the use of longer duration stretches in order to provide enough stimulus to prevent a formation of muscle contractures [73].

Research efforts thus far have not determined any specific guidelines for the use of stretching for the treatment of spasticity, most likely due to the heterogeneity of methodologies: subjects and the level of their impairments and a variety of stretching interventions and outcome measures [99]. There are only a few studies examining the effects of stretching on spasticity that specifically results from SCI. Odeene and Knutsson studied paraplegic patients with ankle spasticity and found that a single 30 minute stretch (maximal dorsiflexion) significantly reduced resistance to passive movement $(17 \%$ reduction) as measured by sinusoidal ankle movements at three different velocities immediately after the intervention. In the same study researchers examined stretching (15 degree dorsiflexion) while weight bearing ( $70 \%$ body weight) for 30 minutes and found a reduction in resistance to passive movement by $26 \%$. In the discussion authors suggested that decreased spasticity resulting from their interventions can last for several hours 
although no data was reported to support that statement [100]. In another study on the effects of stretch on hip abduction ROM in patients with adductor spasticity resulting from various neurologic impairments (including $\mathrm{SCI}, \mathrm{n}=3$ out of 10 ), Odeene found that a single 30 minute adductor stretch administered with a mechanical device resulted in significantly increased passive and voluntary abduction ROM. From the EMG recordings it was also apparent that stretching resulted in decreased antagonist co-contraction during voluntary movement although no quantitative analysis was performed on the EMG data [101]. More recent research on the treatment of spasticity has been conducted in subjects with brain damage following stroke, head trauma and multiple sclerosis. Jo et al., have examined stretching in stroke patients with wrist and finger flexor spasticity. The intervention protocol consisted of a 10 min stretch using a custom made device that placed the wrist and fingers into extension which was applied twice a day, 7 days a week for 4 weeks. The outcome measures were taken 4 weeks prior to stretching intervention, immediately prestretch and immediately after the 4 weeks of stretching and included the Modified Ashworth Scale (MAS) $[102,103]$ for the assessment of spasticity, active ROM and motor function using the Fugl-Meyer (FMA) hand and wrist motor assessment [104]. The authors found that stretching resulted in a significant reduction of spasticity (decrease in MAS scores) and improvement in motor performance (increase in FMA scores) [105]. However, the most objective measure in the study, ROM, was not changed. Given the somewhat subjective nature of the MAS and FMA assessments it is important that the assessments were done by independent or blinded therapists which was not the case in the current study (or at least was not reported). This obvious weakness should be taken into consideration when conclusions are made. A well-designed randomized controlled blinded pilot study 
examined the effects of an arm positioning procedure that consisted of maximal (without pain) shoulder abduction and external rotation with a supinated forearm for 30 minutes twice a day on weekdays for 5 weeks. The main outcome measures consisted of MAS, FMA and ROM. The results of the study demonstrated that subjects allocated to the experimental group had a significantly slowed development of contracture (greater shoulder abduction ROM), however spasticity remained unchanged [106]. Bressel and McNair in their study of 10 stroke patients with ankle stiffness found that 30 minutes of either static or cyclic stretching significantly reduced passive stiffness of the ankle acutely, however, this improvement did not translate to a functional task, at least as assessed by the 10 meter walk time. [107]. Waldman et al., used a robotic system to administer stretching for 20 minutes that was followed by active strengthening exercises for 30 minutes and a 10 minute stretch as a cool down. The protocol totaled 60 minutes of robot assisted training and was done 3 times a week for 6 weeks. Spasticity was significantly reduced as assessed by MAS scores acutely after the intervention, however, at a 6 week (without training) follow up MAS scores returned to pre-training values [108]. In another study the researchers utilized robotic foot plate devise to test a PNF stretching protocol for treatment of spastic ankle in seven stroke patients. The robotic device rotated the ankle to maximal dorsiflexion at which point subjects performed an isometric contraction $(10-50 \%$ of maximal voluntary contraction based on EMG) for 15 seconds for 30 trials. Subjects trained 3 times a week for 3 months. The PNF stretching technique resulted in significant improvements in all outcome measures regarding spasticity including resistance to passive movement, passive ROM and quasi-static stiffness of the ankle joint [109]. The most recent study examined the effects of several interventions on spasticity in patients with SCI. The 
investigators found that stretching, cyclic passive movement and transcutaneous spinal cord stimulation were effective at reducing spasticity. However, only the latter two had lasting effect (45 minutes after intervention) [110] Thus, stretching, especially in combination with weight bearing or strengthening exercises appears to reduce spasticity and, as a consequence, to improve some functional aspects. Based on the studies reviewed here, the effects are observed acutely after intervention and improvements in spasticity disappear without continual therapy. A systematic review has also found positive effects of stretching on spasticity, however, the authors emphasize the need for better quality studies in order to come to a consensus on a paradigm for stretching [99].

\section{Rehabilitation of Locomotor Function after Spinal Cord Injury}

Locomotion is a complex motor task that involves coordination of multiple areas of the central nervous system, musculoskeletal system as well as peripheral nervous system. Within the CNS the key structures for planning, initiation and control of locomotion include cortex [111], basal ganglia [112, 113], and mesencephalic locomotor region (MLR), specifically the cuneiform nucleus (CN) and pedunculopontine nucleus (PPN) $[114,115]$. Brainstem locomotor regions that receive projections from the higher brain areas give rise to descending tracts (upper motor neurons) such as reticulospinal, rubrospinal and vestibulospinal tracts which are important for modulation of excitability of locomotor circuitry in the spinal cord, as well as postural stability [114]. The lumbar enlargement of the spinal cord contains the alpha motoneurons that synapse onto the effector organs-hindlimb skeletal muscles. Alpha motoneurons are organized into motoneuronal pools based on the muscle and their function (flexor vs extensor) as well as muscle synergies $[116,117]$. A complex network of interneurons known as the central pattern generator $(\mathrm{CPG})$ controls the orderly recruitment of these pools and is responsible 
for coordinated inter and intramuscular activation needed for smooth locomotion [118, 119]. At the onset of locomotion proprioceptive neurons located in the muscles, joints and skin become activated and send feedback to the lumbar locomotor circuitry and modulate ongoing activity [120].

It is now well established that the lumbar circuitry of the spinal cord contains a network of interneurons that produce a rhythmic and patterned motor output which drives the activation and coordination of muscles to produce locomotion, termed CPG $[118,119$, 121]. Our understanding of the contribution of this critical component for locomotor function has evolved throughout the years of research. In early 1900s Sherrington documented the ability of animals with complete transections in the thoracic spinal cord to generate stepping. Sherrington postulated that spinal stepping was a result of ongoing integration and propagation of flexion and cross extension reflexes [122]. Later, Graham Brown showed that a spinal cord isolated from supraspinal and peripheral inputs could alone produce rhythmic and patterned neuronal activity and concluded that the spinal cord contains a neuronal networks sufficient for the production of locomotor-like output [123]. G. Brown's finding however, remained unnoticed for a few decades and the view of sensory dependent reflex-driven locomotion persisted until the evidence for central pattern generators (CPG) within the neural circuits of invertebrate animals began to emerge [124]. In 1960, Hughes and Wiersma found that the abdominal ganglia of crayfish isolated from peripheral inputs maintained rhythmic bursting that produced appropriately patterned swimmeret movement similar to that generated during intact swimming behavior [125]. In 1961, Wilson studied flight control in locust and demonstrated that although sensory feedback was important for modulation of certain properties of neuronal activity, 
deafferentiation did not significantly impact the pattern of oscillations evoked by electrical stimulation of the brain and it was remarkably consistent with the pattern during actual flight [126]. In the 1970s, more evidence emerged suggestive of intrinsic capabilities of the isolated central neuronal networks to produce rhythmic patterns independent from sensory inputs in other invertebrate models $[127,128]$. A significant step towards wide acceptance of the concept of CPG-driven locomotor behavior was made by Cohen and Wallen when they demonstrated fictive swimming using an in vitro spinal cord preparation from the lamprey [129]. Rossignol et al., provided exceptional evidence for a CPG in the mammalian spinal cord using a cat model of fictive locomotion [130]. Overall, it took 50 years for the scientific community to accept the CPG-based perspective on locomotion after the first experimental evidence of the capabilities of intrinsic spinal networks to produce locomotor-like patterns [124].

Discovery of the CPG gave rise to the number of research efforts to dissect the functional circuitry underlying locomotion. The organization of the CPG network is one of the key remaining questions in our understanding of locomotion. The earliest speculations on CPG organization came from Graham Brown's work when he made his original observation of intrinsic capacity of the isolated spinal cord to produce locomotor-like pattern. He proposed an idea of two "half-centers" for organization of the rhythmic neuronal networks, in which each half center excites either flexor or extensor motorneurons and that the half centers have mutually inhibitory actions onto one another [123]. This theoretical set-up was schematically shown by Jankowska et al, who then also provided the first empirical evidence suggestive of the half centers concept. Using intracellular recordings together with bilateral stimulation of flexor reflex afferents in spinal cats 
injected with L-3,4-dihydroxyphenylalanine (L-DOPA), Jankowska et al demonstrated the presence of interneurons that mediate reciprocal inhibition between ipsilateral and contralateral flexors and extensors $[131,132]$. The half center concept for the control of locomotion still stands and is built upon with emergence of new evidence from studies on the CPG network. It is now generally accepted that each limb has its own half center (or $\mathrm{CPG}$ ) based on the observations that the left and right legs can maintain independent rates in humans and in spinal cats while walking on a treadmill [133-135]. Existence of circuitry for intralimb coordination contained within the spinal cord is demonstrated by experiments in cats that show persistence of coordination between two hindlimbs when the thoracic spinal cord is transected [135] or between forelimbs and the hindlimbs in cats with high cervical lesions [136]. In an effort to combine various observations in CPG research, Rybak et al., have proposed a computational model for the CPG [137]. The insights from studies of fictive locomotion in the cat, specifically spontaneous omissions of activity (deletions) and the effects of afferent stimulation on a locomotor rhythm have given rise to a model with a two-level CPG in which rhythm generation $(\mathrm{RG})$ is separate from pattern formation (PF) $[138,139]$. According to this model, the RG centers drive the activity within PF network, which then distributes the rhythm and defines the pattern for the motoneuronal activation.

Part of the excitement about the identification of mammalian spinal locomotor CPG is due to the potential clinical relevance that this finding holds. If circuitry necessary for the generation of locomotion is in fact located within the lumbar spinal cord which is spared in the majority cases of SCI then it becomes feasible that with proper activation or modulation of these networks people with severe SCIs can have a hope for restoration of 
their locomotor function. However, skepticism persists regarding a locomotor CPG in humans. Although it is not feasible to directly test for CPG in the human spinal cord, for example, with intracellular recordings due to the obvious invasive nature of these methods, the indirect evidence for the presence of a locomotor CPG in humans is substantial. First, given the strong evidence for CPG in every mammalian spinal cord tested and the fact that the spinal cord is one of the most well preserved structures phylogenetically it is more logical to argue for the existence of the CPG in humans than against it $[119,140]$. Second, human infants (3-6 months old) can generate "stepping" before the emergence of locomotor behavior given an appropriate proprioceptive stimulus $[133,141]$. Since the descending brain inputs are very immature at birth and do not reach the levels of myelination comparable to that of the spinal tracts in the adult humans until the age of 2 [142], researchers believe that stepping in children below the age of 1 is evidence of spinal centers for locomotion [133]. Furthermore, there have been several reports of locomotorlike activity in the legs of patients with both complete and incomplete SCI. Calancie et al., documented robust involuntary stepping-like leg movements in a chronic (17 years post SCI) patient with cervical SCI that could be reliably evoked by extension of the hips [143]. Several years later the same author reported additional cases of spinal myoclonus. A patient with an incomplete lesion displayed involuntary leg activations with the pattern characteristic of rhythmic stepping, while in 4 patients with complete SCIs hip extension evoked bilateral co-contractions in all leg muscles, and although the pattern was not reciprocal as occurs in stepping, the frequency of EMG bursting was similar to subjects with incomplete injuries. The authors concluded that these rhythmic movements were a result of CPG activation [55]. Perhaps the most compelling evidence for existence of CPGs 
in the human spinal cord was provided by Dimitrijevic et al. The authors used epidural stimulation of lumbar spinal cord to evoke stepping-like EMG activity in the leg muscles of six subjects with complete paraplegia. From their study it became clear that the neuronal circuitry responsible for shaping locomotor output resides within the lumbar spinal cord but due to the severed supraspinal pathways and, therefore, lack of excitatory modulation, remains dormant. Epidural stimulation was able to replace the very basic function of the brainstem pathways in that it provided enough excitability to the locomotor CPG to induce stepping-like movements which could then be further modulated by the afferent input from the legs [144]. The attempts to access spinal locomotor centers have been made also in intact adult human subjects. Using various modes of activation several groups have been successful at eliciting automated locomotor-like stepping in suspended limbs of healthy subjects using stimulation of peripheral structures (ex. muscle vibration, nerve stimulation) $[145,146]$ or more direct spinal electromagnetic stimulation [147]. Although we may never be able to directly prove the existence of CPG in humans, the accumulating indirect evidence points towards a far more capable spinal cord circuitry than previously considered and thus gives hope to researchers, therapists and patients with SCI to bring about improvements in locomotor function given that we continue to learn the new ways of properly engaging the key locomotor circuitry.

Preservation of the CPG is advantageous to higher mammals, since presence of automated circuitry for such complex tasks as locomotion which requires coordination of muscle activity between joints and limbs frees the higher brain centers to engage in other tasks. On the other hand, supraspinal and afferent modulation that the CPG is also required for the willful, directed and accurate expression of locomotion. Severe SCIs result in partial 
or more rarely complete loss of supraspinal modulation of the spinal locomotor circuitry, whereas afferent input remains intact. An outstanding demonstration of the capacity of CPG for adaptive plasticity by Rossignol et al., capitalizes on the importance of the afferent input after SCI [121]. In their experiments the hindlimbs of cats with a complete spinal cord transection at the thoracic level 13 were trained to step on the treadmill, while the forelimbs were placed on a stationary platform. After only 3-4 weeks of training the cats were able to generate fully weight supported stepping. Pre- and post-transection kinematics of the hindlimb stepping as well as EMG patterns were highly comparable to each other, although some deficits persisted after spinalization despite the training (ex. decreased step length, paw drag at swing onset, etc) [148]. Such a remarkable observation did not only demonstrate the potential of the CPG for adaptive plasticity driven by appropriate afferent input but also, together with other animal $[149,150]$ and human studies [151], provided a rationale for implementation of locomotor training in the rehabilitation of SCI patients $[134,152]$.

For locomotor training, SCI patients are placed in a harness to provide partial weight support over a treadmill. While the treadmill is moving, physical therapists either assist the stepping or completely move the legs for the patient depending on the severity of impairment $[151,153,154]$. Robotic devices, such as the Lokomat have also been used to assist the step training [155]. The duration, number of sessions per week and length of overall body weight supported treadmill training (BWSTT) program that has been investigated in studies varies, but generally consists of 30-60 minute sessions, 2-3 times a week for 4-8 weeks [156]. Many studies have investigated the effectiveness of BWSTT at improving function in patients with SCI. Generally, there is evidence that locomotor 
training improves function of patients with incomplete injuries or according to American Spinal Injury Association scale - C and D (ASIA C and D) injuries that have some ambulatory capacity. For example, a single session of BWSTT (10-20 minutes in duration) in a small study with 4 subjects with incomplete SCI (ASIA D) resulted in $26 \%$ and $25 \%$ improvements in self-selected and maximal speed in overground locomotion, respectively [157]. Behrman and Harkema, reported studies on 3 subjects with incomplete SCIs (two ASIA D and 1 ASIA C) and one subject with a complete (ASIA A) SCIs. Treadmill and overground locomotor training were carried out over several weeks. The three subjects with incomplete injuries had improvements in overground walking that translated to increased mobility in their activities of daily living (ADL). The subject with a clinically complete injury achieved independence in stepping on the treadmill and had increased weight bearing capacity which did not translate to overground locomotion [153]. In a larger clinical trial 64 subjects with incomplete SCI (ASIA C and D) were randomly allocated to one of the four locomotor training protocols: overground training with electrical stimulation (ES), treadmill with manual assistance, robot-assisted treadmill or treadmill with ES. The authors found that there was an overall effect of training on overground walking speed over 10 meters and distance walked in 2 minutes. There were no significant differences between groups in speed improvement. However, the gains in the distance covered as well as the effect size of distance and speed were greater in the overground training group as compared to all the treadmill training groups, a finding most likely attributable to an established phenomenon of task-specific adaptation [158]. Ten subjects on follow up at least 6 months after the training had decreased walking speed which was still faster than prior to training suggesting some retention of functional improvements 
[159]. Patients with clinically complete SCIs that undergo BWSTT improve certain aspect of locomotor function most evident in EMG characteristics such as pattern, including the reciprocal activation between flexors and extensors, interlimb coordination and EMG amplitude $[160,161]$. Unfortunately, these improvements do not translate to overground locomotion [134, 162] and decline after cessation of locomotor training [163]. Given that the majority of SCI cases are characterized as clinically complete injuries (ASIA A), the ineffectiveness of BWSTT at improving locomotor capacity in this patient population has been disappointing.

The effectiveness of treadmill training at improving locomotor capacity of animals has set high expectations for BWSTT. However, the gains from hours of intensive BWSTT were rather modest. The shortcomings in translation made scientists look back at the evidence for potential explanations of the poor outcomes $[162,164]$. In a recent review, de Leon and Dy reexamined the popular experiments which demonstrated an amazing capacity of spinalized cats to generate almost normal locomotor patterns after a few weeks of treadmill training. The authors point out some overlooked and/or unreported observations that could explain why BWSTT has not been as successful as expected in the clinic. For example, when the same spinal animals that stepped beautifully on the treadmill were placed overground or in their cages they defaulted to propelling themselves forward using their forelimbs while hindlimbs dragged behind. Although occasional stepping did occur if the hindlimb happened to be in a weight bearing position, most of the time the cat's hindlimbs did not (or could not) generate consistent weight supported stepping overground. The authors explore the reasons for such observation, highlighting the fact that motor learning is task-specific and treadmill stepping did not train some of the key aspects 
for overground locomotion such as lateral spine stability and balance, hindlimb-forelimb coordination and voluntary control of locomotion [164]. Knowing that spinal cats still fail to walk overground after hours of intensive treadmill training makes the same finding in humans less surprising. However, the question remains as to how we can harness the undeniable capability of the spinal CPG for adaptive learning and translate that to functional improvements in patients with SCI? The answer to that question may slowly come to light as we gain understanding of both animal and human spinal cord organization and its changes after SCI. For example, it has become more apparent that human spinal locomotor networks depend on supraspinal connections for maintenance of excitability much more than the CPG in rodents [162]. Rats with SCI have robust locomotor recovery given appropriate afferent input [165], whereas the CPG in patients with severe chronic SCI responds to proprioceptive feedback at the onset of BWSTT seen as locomotor-like EMG activation in leg muscles, but fails to maintain the responsiveness as EMG activity ceases shortly (10-12 minutes) into a locomotor training session [166], the phenomenon called EMG exhaustion [167]. The mechanisms responsible for the diminishing motor output during locomotor training are unknown, but predominance of the inhibitory influences of locomotor patterns have been proposed due to lack of supraspinal drive [166].

Since modulation of human CPG by afferent inputs does not result in as large of functional gains as in animal models of SCI, other ways of modulations apparently are required for significant improvements. A very powerful demonstration of the human CPG capacity was made by Angeli et al., when four paraplegic patients (two ASIA A and two ASIA B) implanted with epidural electrical stimulators over the lumbar spinal cord were 
able to stand, generate stepping patterns over the treadmill and even produce voluntary leg movements unattainable prior to epidural stimulation for many years after the SCI in these subjects [168]. Exact mechanisms by which epidural stimulation (ES) was able to elicit these improvements in motor function are unknown. The authors speculate, however, that since voluntary movements on command emerged shortly after the beginning of ES (4-11 days) and extensive plasticity of networks in this time period is unlikely, ES by altering spinal network excitability accentuated central excitatory drive through some supraspinal connections that apparently remained in these patients and increased the responsiveness of local spinal interneurons to both supraspinal and afferent inputs. Step and stand training in combination with ES further improved the voluntary ability to execute movement which translated to increased function in the activities of daily living [168]. Since this breakthrough study, other less invasive methods for altering spinal cord excitability to enhance its functional capacity have been proposed. Transcutaneous electrical stimulation (tES) over the lumbar spine was first used to elicit locomotor-like activity in non-injured subjects placed in gravity-neutral position to provide further evidence for human CPG [169]. Recent studies have begun exploring this finding in its clinical application for subjects with severe SCI and have already yielded promising results. First, the ability of tES to evoke locomotor-like movements were confirmed in patients with motor complete SCI. Second, voluntary effort from the subjects modulated this ongoing activity and increased its robustness. Third, over the course of 4 week of tES and conditioning without stimulation by passively moving the legs in step-like fashion subjects improved their voluntary ability to initiate and maintain step-like movements even in the absence of stimulation [170]. 
These exciting findings demonstrate that human spinal locomotor networks in fact are capable of plasticity in activity-dependent manner. Unlike animal locomotor CPG that through provision of appropriate afferent input readily regains its ability to generate locomotion, human CPG function appears to be much more reliant on excitatory drive normally provided by supraspinal connections. In the presence of SCI the human CPG can be reactivated and/or successfully modulated by electrical stimulation to the spinal circuitry. The future of locomotor rehabilitation lies in the further investigation of the potential of electrical stimulation in combination with training, technological advancement and gains in understanding of the physiology and pathophysiology of locomotor neuronal networks after a SCI.

\section{Effects of Hindlimb Muscle Stretching on Locomotor Function in SCI rats}

Unlike humans, rats spontaneously recover locomotor function even after a severe incomplete SCI. The stepping rats generate after a $\mathrm{SCI}$ is obviously impaired, as there is persistent lack of coordination between forelimbs and hindlimbs, trunk instability, toe drags and frequent to occasional dorsal stepping. Nevertheless, the animals are able to maintain frequent to consistent weight support during stepping. If such levels of functional recovery could occur in humans with SCI it most likely would increase the patient's independency and quality of life. The overarching theme in our laboratory ("Exercise fixes everything") is derived from a belief that the discrepancy in functional outcomes following a human verses an experimental SCI in the animals depends on more than just the difference in the CNS organization. We believe that the state of activity immediately after the injury has an impact on the course of recovery as well. In humans SCIs most commonly occur in motor-vehicle accidents [1] and are accompanied by other trauma often making patients bed-ridden for weeks at a time. Once the patients with a severe SCI are released 
from the hospital they become dependent on a wheelchair for mobility. On the other hand, after an experimental SCI rats are placed in their cages and within short period of time the animals become active, moving as they can. They first use their forelimbs to drag themselves through the bedding. As spinal shock resolves the return of reflexes facilitates hindlimb involvement in locomotion. Using an overnight activity recording set up in the lab we have found that the animals shortly after injury can travel up to 150 meters a night in their cages. By 3-4 weeks post-SCI animals basically retrain themselves achieving significant locomotor recovery. In order to test the hypothesis of in-cage activity-driven locomotor recovery in rats, previous student, Krista Caudle, designed an experiment in which rats with moderate SCIs were placed into custom-designed wheelchairs overnight during the most active hours of the rats beginning 1 week post-injury. As hypothesized, rats whose hindlimbs were restrained in wheelchairs overnight for several weeks did not achieve the same locomotor recovery as the non-wheelchair control animals [165]. In addition to this excellent observation the authors of the study noticed that wheelchair animals developed significant muscle atrophy and contractures around the immobilized joints. In order to treat and care for the animals a clinically relevant hindlimb stretching protocol was developed with help of physical therapists from Frazier Rehabilitation Institute who work with SCI patients on the daily basis. The protocol consisted of six stretches for the major hindlimb muscle groups applied bilaterally: ankle, knee and hip flexors and extensors, as well as hip abductors and adductors. Each stretch was held statically for 1 minute amounting to a 12 minute session which was repeated twice and thus each animal received 24 minute of stretching per day. This protocol was performed for 5 days on weekdays for 8 weeks. The locomotor function of the animals was assessed using 
Basso, Beattie, Bresnahan Open Field Locomotor Scale (BBB) three times a week: Monday am (pre-stretch), Monday pm (after one stretching session) and Friday pm (after 5 days of stretching). In addition biweekly walking kinematics assessing 2 hindlimb segments across 3 joints were recorded. Stretching had no effect on locomotor function of wheelchair animals and neither did it improve the passive ROM in the joints affected by contractures. The most unexpected finding, however, was that non-immobilized SCI animals that received daily stretching had reduced locomotor function to around the same level as the wheelchair-immobilized rats [165]. Given that stretching was initially implemented for treatment of contractures in accordance with regular clinical practice this finding was alarming and motivated further investigation. In a separate study Caudle et al., focused on the effects of stretching on the locomotor function of rats with moderate SCI. The same stretching protocol was administered to the rats beginning at 4 days post-SCI for the following 8 weeks. Consistent with previous observation, stretching beginning acutely after a moderate SCI significantly impaired locomotor recovery of the animals as measured by BBB Open Field Locomotor Scale, kinematics and swimming assessment [171]. These two studies laid the foundation for the stretching projects in our laboratory and raised additional questions which, when answered, will further our understanding about the stretching phenomenon and its clinical relevance. First, does stretching have the same negative effect on the locomotor function in animals with more clinically relevant moderately-severe SCIs? What is the effect of stretching protocol on locomotor function in rats with chronic SCIs, whose locomotor function has stabilized in a plateau for multiple weeks prior to stretching? Would stretching be detrimental to the locomotor function if instead of holding each stretch at the end ROM for 1 minute statically, it would be 
administered in a more dynamic fashion? What is the clinical relevance of the stretching phenomenon? Finally, what are the physiological mechanisms underlying the stretchinduced locomotor deficits? Based on the existing literature and specifically work by Grau et al., [172] we first, wanted to investigate the role of nociceptive afferents in mediating the negative effects of stretching on locomotor function. Therefore, the goal of the following thesis is to answer the questions stated above.

\section{Specific Aims and Hypotheses}

\section{Specific Aim 1:}

a. Determine if our stretching protocol is detrimental to locomotor function in animals with more clinically relevant moderately-severe spinal cord contusion injury.

b. Determine if our stretching protocol starting at the chronic time point after spinal cord injury ( 10 weeks) is detrimental to locomotor function in rats with moderatelysevere SCI.

c. Determine if a dynamic stretching protocol is detrimental to the locomotor function in rats with moderate spinal cord injuries.

Hypothesis $(a \& b)$ : Static stretching will have detrimental effects on locomotor function in rats with moderately-severe spinal cord injuries at both acute and chronic time points.

Hypothesis (c): Dynamic stretching will not be detrimental to locomotor function in rats with subacute mild-moderate spinal cord injuries.

\section{Specific Aim 2:}

Determine the degree to which EMG responses to stretching in rats resembles those seen in human. 
Hypothesis: Rats will have EMG patterns (clonic-like muscle contractions, spasms, etc) in response to stretch that are similar to those in patients with SCI.

\section{Specific Aim 3.}

Determine if the activation of nociceptive afferents contributes to stretch-induced locomotor deficits.

Hypothesis: Systemic depletion of capsaicin-sensitive nociceptive afferents will result in significant attenuation of stretch-induced locomotor deficits in capsaicin treated stretched animals. 


\section{CHAPTER III \\ DISRUPTION OF LOCOMOTION IN RESPONSE TO HINDLIMB MUSCLE STRETCH AT ACUTE AND CHRONIC TIME POINTS AFTER A SPINAL CORD INJURY IN RATS}

\section{Introduction}

Severe but incomplete SCI results in partial paralysis below the level of injury due to the loss of descending inputs onto voluntary motor and the more automated locomotor circuitry of the spinal cord. Partial paralysis, in turn, results in prolonged periods of immobility accompanied by significant alterations in the musculoskeletal components [65]. Over time, the spinal cord circuitry below the level of injury undergoes a number of adaptations that lead to an increase in alpha motoneuron excitability $[173,174]$. This increase can be associated with improvements in locomotor function $[173,175]$. However, the system lacks appropriate modulation by inhibitory circuitry due to impaired control from supraspinal centers [176-178] and local maladaptive plasticity [63]. These changes combine to result in exaggerated motor output (hypertonia and spasticity) in response to muscle stretch and/or other sensory input [166]. As a result, approximately $78 \%$ of patients with chronic SCI develop some level of spasticity [46]. Although spasticity has potential benefits for some patients, indirectly contributing to their ability to stand or perform daily tasks such as transfers, it also presents a multitude of unwanted consequences that presumably contribute to over half of the patient population with spasticity reporting it as a major obstacle to resumption of activities of daily living [179]. Unmanaged spasticity, 
where muscles remain in shortened positions for prolonged periods can lead to the development of joint and muscle contractures that manifest as dramatically decreased ROM [180] about affected joints. Preservation of a functional ROM is not only important for timely initiation of rehabilitation [66] but also can significantly improve independence in some patients with SCI. For example, an elbow contracture in patients with cervical level 6 injury rendered them unable to perform transfers and maintain bed mobility functionally making them similar to patients with cervical level 5 injury.

Stretching remains the cornerstone for the treatment by physical therapists of both spasticity and muscle contractures $[179,181,182]$, and is encouraged even in the absence of contractures in order to maintain soft tissue extensibility because it is believed that preventing contractures is easier than treating them [73]. Stretching provides a potent mechanical stimulus for the induction of protein synthesis [183] and has been shown to result in serial addition of sarcomeres within muscle fibers [68] which can potentially prevent atrophy [184] and decreases in muscle length. These observations suggest that muscle stretch should be an effective method for achieving desirable changes in muscle length, or preventing undesirable changes in soft tissues and thus the rationale to include stretching therapy in the rehabilitation program for patients with SCI appears sound. However, evidence that most commonly employed stretching techniques actually improve symptoms of spasticity, ROM and/or prevent contracture formation in subjects with SCI is mixed at best [5].

Previously, we found that wheelchair hindlimb immobilization in rats with mildmoderate spinal cord injuries resulted in the loss of locomotor function and in addition, some animals developed contractures, grossly similar to human patients [165]. Thus, we 
invited physical therapists who work with human SCI patients to guide us in the development of a clinically relevant stretching protocol for the treatment and prevention of contractures in hindlimb-immobilized animals as part of their daily care. The protocol was standardized for stretching muscles around the major hindlimb joints: ankle flexors/extensors, knee flexors/extensors, hip flexors/extensors and hip adductors/abductors. Surprisingly, when the same stretching protocol was applied to control non-immobilized injured rats, we found that it caused a decrease in their locomotor function [165]. The goal of the current study was to extend these observations, using the same stretching protocol, to a more clinically relevant moderately-severe spinal cord contusion model and to examine the effects of stretching at both acute and chronic time points. Based on our previous findings, we hypothesized that stretching would have detrimental effects on locomotor function in rats with spinal cord injuries at both acute and chronic time points.

\section{Materials and Methods}

\section{Spinal Cord Injury and Study Design}

Twenty-two adult female Sprague-Dawley rats (190-230g) were utilized in the study. All experimental procedures involving animals were approved by the University of Louisville Institutional Animal Care and Use Committee. Upon arrival, animals underwent a week-long acclimatization protocol that involved daily gentling and introduction to the experimental apparatus used for kinematic and behavioral assessments. Figure 1 shows a timeline for the entire experiment, including collection of the primary outcome measures. Rats received a T9 laminectomy followed by a T10 moderately-severe spinal cord

contusion (25 g/cm, NYU Impactor) as described previously [185]. Animals were randomly assigned to one of three experimental groups after the first behavioral locomotor 
assessment on day four post-injury: Acute Stretch (AcS, n=10), Chronic Stretch (ChS, n=9) or injury control for muscle histology $(\mathrm{n}=3)$. The stretching protocol was implemented as previously described [186] for the AcS group starting at 4 days post-injury and continuing for 5 weeks and for the ChS group starting at 10 weeks post-injury and continuing for 4 weeks. Stretching in the $\mathrm{ChS}$ group was stopped after 4 weeks due to the development of severe contractures in some of those animals and the inability of our physical therapists (PTs) to achieve full end ROM during stretching. Briefly, the stretching protocol consisted of two 12-minute sessions of 6 static stretches (each held for 1 minute at the end ROM) performed bilaterally of major hindlimb muscle groups: ankle flexors \& extensors, quadriceps, hamstrings and hip abductors/adductors (each muscle group received 2 minutes of stretch per day). There were seven PTs who participated in stretching sessions. Animals were rotated among the PTs such that no animal was stretched twice by the same therapist in any given week. PTs were trained on proper animal handling and hindlimb positioning for each stretch in a pilot study that is not reported. We did not measure the forces applied during stretching, thus physical therapists were instructed to closely monitor the limb position and to achieve normal end ROM for each stretch. Stretching was carried out 5 days a week (Monday-Friday). After 5 weeks of stretching the AcS group survived for an additional 13 weeks, and the ChS group for 7 weeks. 


\section{Figure 1}

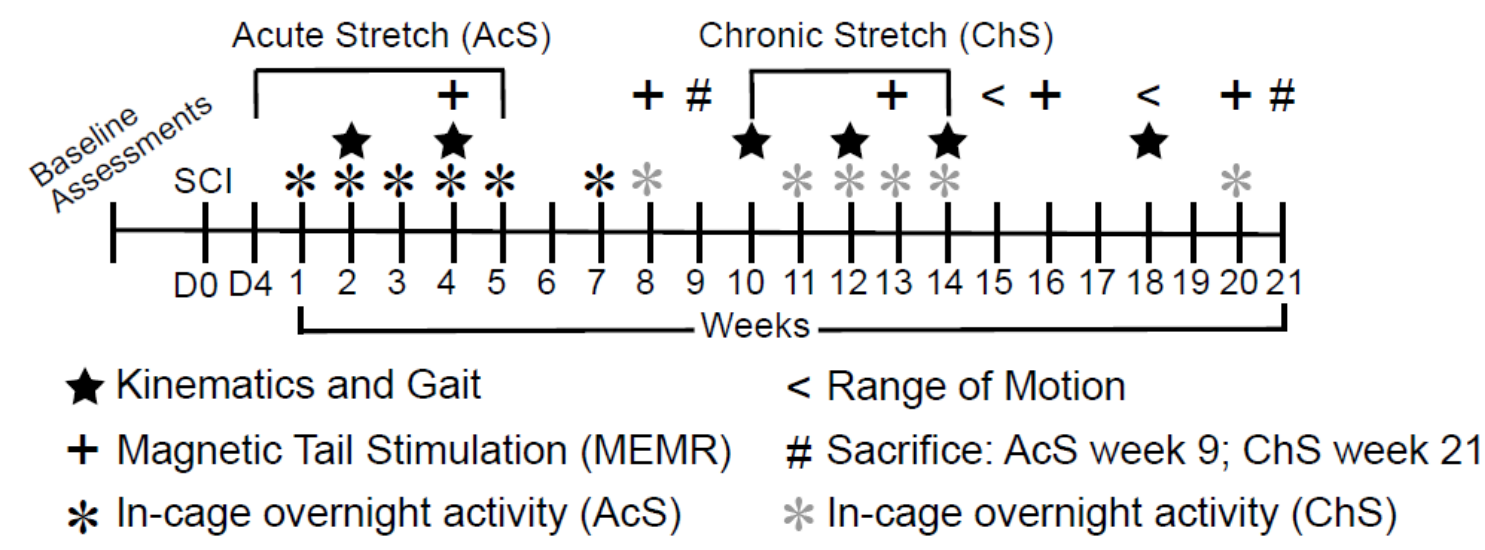

Figure 1. Time-line indicating the primary events of the acute and chronic stretch study including the outcome measures and weeks of stretching relative to the time of injury 


\section{Locomotor functional assessments}

Overground stepping was assessed using the BBB Open Field Locomotor Scale [187] as previously described. BBB assessments were performed 3 times a week: Monday am (pre-stretch), Monday pm ( $>1$ hour post-stretch) and Friday pm ( $>1$ hour post-stretch) during the weeks of stretching and once weekly (Monday am) when stretching ended. During the first 5 weeks the ChS group served as a behavioral control for the AcS group. The BBB scores of the $\mathrm{ChS}$ group plateaued at 4 weeks post-injury and they served as their own controls. In addition, hindlimb kinematics and gait were assessed using digital video (sagittal and ventral views) acquired while the animals stepped in 2 " $(5 \mathrm{~cm})$ of water sufficient to supply approximately $60 \%$ body weight support, as described previously [188]. We used a 3-segment, 2-angle model of the hindlimbs for kinematic analysis with the hip-ankle-toe (HAT) representing ankle and knee excursions and the iliac crest-hipankle (IHA) representing hip and knee excursions. Gait analysis was derived from ventral view videos that allowed accurate identification of paw placement and timing, and each paw placement was determined to be plantar (paw and toes appropriately placed with dorsal surfaces up) or dorsal (with one or more toes curled and/or the paw itself oriented such that dorsal surface was observable in the ventral view). Three indices of gait were determined: the central pattern index (CPI), calculated as the number of correctly patterned plantar and dorsal steps over the total number of steps, the regularity index (RI), calculated as the number of correctly patterned plantar steps over the total number of steps (dorsal and plantar) and the plantar stepping index (PSI), calculated as number of hindlimb plantar steps over the total number of forelimb steps. Kinematic and gait analyses were performed 
every other week using MaxTraq \& MaxMate software (Innovision Systems Inc., Columbiaville, MI) and custom designed Excel macros $[185,186]$.

Nocturnal in-cage Activity Recordings

Overnight in-cage activity was measured once a week (Thursday) during the weeks of stretching using overhead cameras (Basler, acA645-100gm) and infrared lights. A twocentimeter tracking dot was drawn with a Sharpie pen on the shaved lower back of each animal. The recordings were made using custom software that acquired high-resolution video at $4 \mathrm{~Hz}$ for 1 minute out of every 10 for 12 hours. The video recordings were analyzed using MaxTraq software and the distance travelled determined using a custom-designed excel based add-in program. Discreet movements of the tracking dot that were less than $2.0 \mathrm{~cm}$ were not counted thus removing much of the movement associated with grooming and sleeping.

\section{Magnetically Evoked Potentials from Tail Stimulation}

Previously, it was established that stretching affects both the mechanical properties of muscles and motoneuron excitability [189]; 30 sessions of stretching resulted in a temporary reduction in H-reflex amplitude[190]. We thus wanted to determine if our stretching protocol similarly affects the excitability of the gastrocnemius motoneuron pool after a spinal cord injury. Unanaesthetized animals were restrained on a pine board using a cloth stockinette as previously described [191]. Afferents in the base of the tail were stimulated using a 25mm figure-8 magnetic coil attached to a MagStim 200 (MagStim Ltd., Whitland, U.K.); stimuli were delivered at $80 \%$ of maximum intensity, sufficient to induce a plateau response but avoid direct muscle/motor axon activation [171]. Gastrocnemius muscle responses (EMGs) were recorded bilaterally using 26-gauge needle electrodes 
[186, 191]. EMGs were analyzed for onset latency and peak-to-peak amplitude. The assessments were carried out every four weeks. During the weeks of stretching, the tail stimulation was performed at least 1 hour after the last stretching session for each animal.

\section{Histological Procedures}

Animals were overdosed with a ketamine $(50 \mathrm{mg} / \mathrm{kg}) /$ xylazine $(0.024 \mathrm{mg} / \mathrm{kg}) /$ acepromazine $(0.005 \mathrm{mg} / \mathrm{kg})$ cocktail and transcardially perfused with phosphate buffer, followed by 4\% paraformaldehyde (PFA) [192]. The spinal cord and hindlimb muscles (Biceps Femoris, Gastrocnemius and Tibialis Anterior) were dissected out, post-fixed in 4\% PFA overnight and cryprotected with $30 \%$ sucrose. The fixed spinal cords were carefully examined to confirm the injury level (T10) and one centimeter long pieces containing the injury epicenter were prepared and placed in tissue freezing medium. Transverse sections were cut at $30 \mu \mathrm{m}$ and stained for spared white matter using eriochrome cyanine (EC) [191]. Photomicrographs were acquired at 4X magnification. Cross-sectional area of compact, darkly stained white matter was traced and measured using ImageJ software (NIH) as described previously [16]. Muscles were divided in two at the mid-belly, placed in tissue freezing medium and transverse sections were cut at $18 \mu \mathrm{m}$. Sections were stained with Masson Trichrome for collagen as a marker of tissue fibrosis and Hematoxylin and Eosin $(\mathrm{H} \& \mathrm{E})$ for identification of centralized nuclei as a marker of regenerating muscle fibers. For collagen quantification, two 10X images were taken from midbelly sections in specified areas oriented to consistent vascular landmarks (branches of posterior tibial artery) in the posteromedial portion of the TA muscle (or to fascia separating the two heads of the gastrocnemius and $\mathrm{BF}$ muscles that were present in all animals. Area $\left(\mathrm{mm}^{2}\right)$ of collagen was measured using ImageJ. Manual tracing of 150 random midbelly muscle 
fibers was performed, per muscle, and cross sectional area (CSA) was determined using ImageJ. It was previously established that a sample size of 150 fibers is sufficient to accurately estimate the mean muscle fiber CSA [193]. Muscle fibers with centralized nuclei were counted from three $40 \mathrm{X}$ images and normalized to the total number of muscle fibers analyzed for each animal (reported as a percentage). Histological analysis was done by an individual blinded to the experimental groups.

Range of Motion Assessment

After 4 weeks of stretching therapy it was noted that $\mathrm{ChS}$ animals had reduced ROM about the hip and knee joints. To confirm this observation we measured passive ROM around those joints at week 15 post-injury. The animals were restrained as for stretching and each hindlimb was moved into hip/knee extension/knee flexion until passive resistance was felt. A goniometer was used to measure the joint angles as described previously [12]. ROM assessment was repeated at week 18 post-SCI when locomotor function had recovered to pre-stretch levels.

Statistical Analysis

Data are presented as group means \pm standard deviation. Mixed model repeated measures analysis of variance (RM ANOVA), fixed and random effects, followed by Bonferroni post hoc t-tests were performed on all outcome measures except gait indices. Non-parametric one-sample t-tests were performed to analyze the CPI, PSI and RI in chronic stretch animals and PSI and RI of the acute stretch group. A binomial proportions non-parametric test was performed to analyze CPI in the acute stretch group. Differences between groups and/or time points were considered statistically significant when $\mathrm{p} \leq 0.05$.

\section{Results}

Open field locomotor function assessments 
Stretching caused a drop in the BBB scores of both the Acute Stretch (AcS) and Chronic Stretch $(\mathrm{ChS})$ groups. Overall, RM ANOVA showed significant difference between groups $(\mathrm{F}=27.9, \mathrm{df}=1,12, \mathrm{p}<.001)$ and within group time points $(\mathrm{F}=37.5 \mathrm{df}$ $=20,12, \mathrm{p}<.001)$. During the first two weeks of stretching, the hindlimbs of the AcS group were flaccid and end ROM was achieved easily during the stretching sessions, with a low perceived force being applied by the physical therapists (PTs). However, muscle tone returned by week 3, and the PTs noted that the perceived force required to achieve the desired stretching positions had increased. Figure 2A shows that the BBB scores were apparently more influenced by stretching at weeks 4 and 5, when additional perceived force was used, and a significant difference was observed at week $5(\mathrm{p}<.005)$. Two weeks after the stretching protocol ended the BBB scores of the AcS group recovered to the level of the unstretched $\mathrm{ChS}$ animals, albeit with high variability. Locomotor function of the $\mathrm{ChS}$ group reached a plateau at post-injury week 4 and remained consistent for the next 6 weeks until the start of stretching at 10 weeks post-SCI (Figure 2B). After only one week of stretching, BBB scores of the ChS group dropped to an average of $0.5(p<.005)$; most animals had only slight movement of one joint on one side. By week 13, all 9 animals had BBB scores of 0 . The vulnerability of locomotor function to stretching at chronic time points was also revealed by the fact that one stretching session (Monday am) was enough to undermine any recovery that took place over the weekend when the animals were not stretched. Within two weeks of the last stretching session, the BBB scores for the ChS group recovered sufficiently to be not different from pre-stretch levels. 
Figure 2

Acute Stretch vs Chronic Stretch BBB Open Field Scores
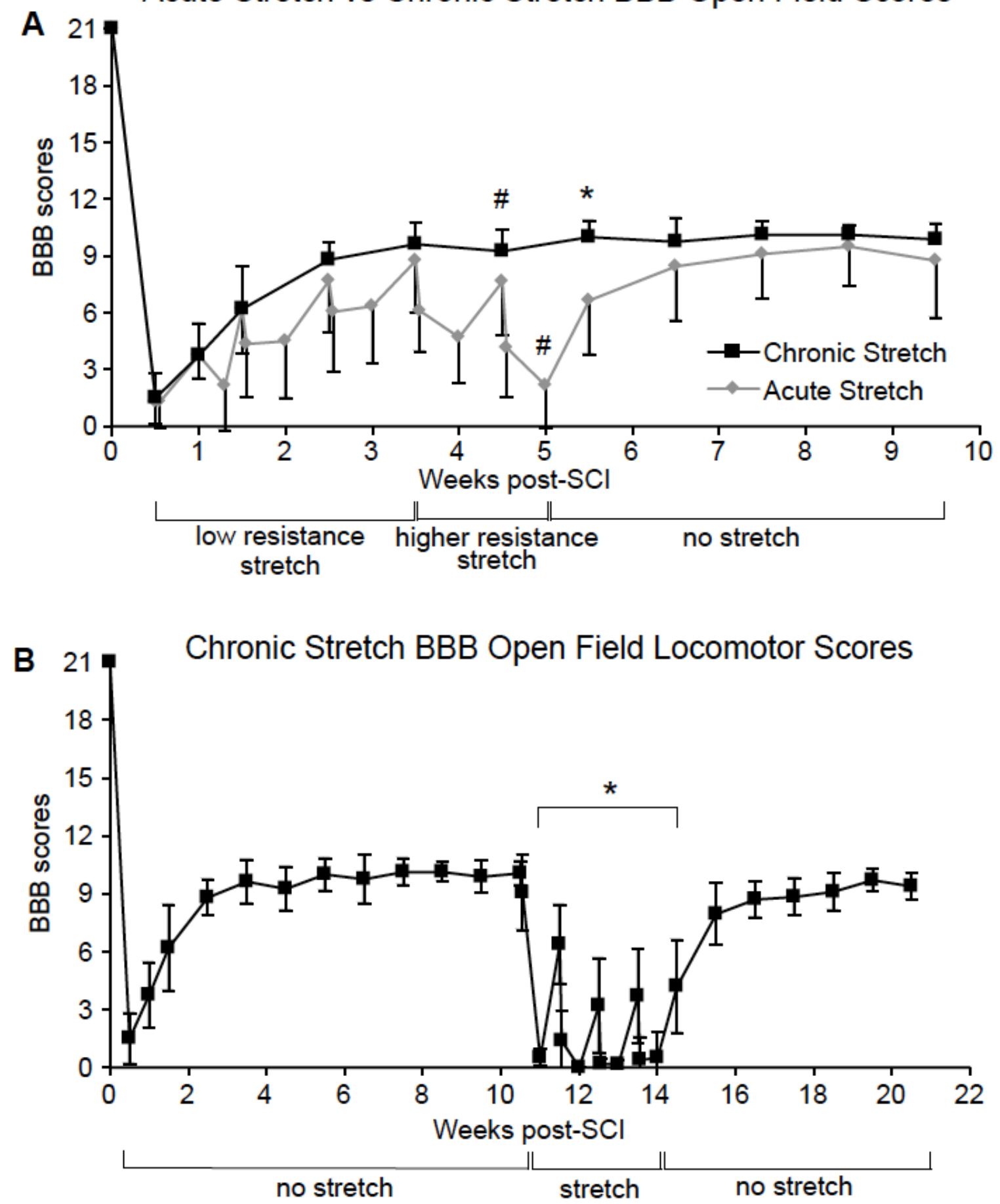
Figure 2. Locomotor function of the animals with sub-acute SCI in response to dynamic stretching protocol.

(A) BBB scores are shown for the acute $(\mathrm{AcS})$ and chronic $(\mathrm{ChS})$ stretch groups over the first 10 weeks post-injury. Drops in BBB scores were modest and not significant during the first 4 weeks but became significant at 5 weeks after higher perceived forces were applied starting at week 4. \# Indicates significant differences between Monday morning and Friday afternoon BBB scores. * Indicates significant differences in BBB scores for stretched and unstretched groups. (B) BBB scores of the $\mathrm{ChS}$ group dropped dramatically after only one week of stretching. * Indicates significant differences between pre-stretch (week 10 Monday morning) and stretch BBB scores. 


\section{Kinematic and gait analysis of the locomotor function}

Kinematic (HAT and IHA excursions) and gait analysis (CPI, PSI, RI) of the AcS animals are summarized in Figure 3A and C. RM ANOVA showed significant time point differences in AcS HAT $(\mathrm{F}=42.7, \mathrm{df}=2,27, \mathrm{p}<.001)$ and IHA excursions $(\mathrm{F}=137.4, \mathrm{df}=$ $2,27, \mathrm{p}<.001)$. Specifically, HAT and IHA “joint" excursions were significantly lower at both week 4 and 8 as compared to pre-SCI baseline $(\mathrm{p}<.05)$. In addition, HAT and IHA excursions were significantly lower at week 4 (during stretching therapy) as compared to week 8,3 weeks after the last stretching session $(\mathrm{p}<.05)$.

At week 4, only two out of ten AcS animals achieved some dorsal stepping in shallow water and thus had a measureable CPI above 0 , the remaining eight did not step and therefore had PSI and RI indices of 0 . By week 8, AcS rats achieved significant recovery revealed by the PSI $(\mathrm{p}<.05)$ and CPI $(\mathrm{p}<.001)$.

Kinematics and gait analyses of the ChS group is shown in Figure 3, B and D. Overall, RM ANOVA showed significant time point differences in ChS HAT excursions $(\mathrm{F}=275.9, \mathrm{df}=4,40, \mathrm{p}<.001)$ and $\mathrm{ChS}$ IHA excursions $(\mathrm{F}=376.1, \mathrm{df}=4,40, \mathrm{p}<.001)$. At week 10 post-SCI, prior to stretching, HAT excursions had recovered sufficiently to not differ significantly from baseline (pre-injury), whereas IHA excursions were still significantly lower $(\mathrm{p}<.05)$. At weeks 12 and 14, during the stretching therapy, ChS animals dragged their hindlimbs resulting in very low angular excursions likely reflecting only passive movements of the ankle as the animal moved in shallow water. Figure 3 shows the significant differences $(\mathrm{p} \leq .005)$ between pre-stretch IHA and HAT excursions (week 10), stretch (12 and 14) and week 18 (4 weeks after the last stretching session) when ChS animals regained locomotor function to a pre-stretch level. 
By week 10 (prior to the beginning of stretching) CPI and PSI for the ChS group recovered to about $50 \%$ of normal, whereas RI remained poor, indicating that both the forelimbs and hindlimbs were achieving plantar stepping, but the two girdles were decoupled (poor or no hindlimb/forelimb coordination). During the weeks of stretching (weeks 12 and 14) RI, PSI and CPI were all equal to zero, indicating a complete lack of stepping, which was significantly different $(\mathrm{p}<.05)$ from pre-stretch (week 10) values. Four weeks after the last stretching session (week 18) $\mathrm{ChS}$ animals regained the ability to step, indicated by the return of RI, PSI and CPI to pre-stretch levels. 
Figure 3

A Acute Stretch: Joint Kinematics

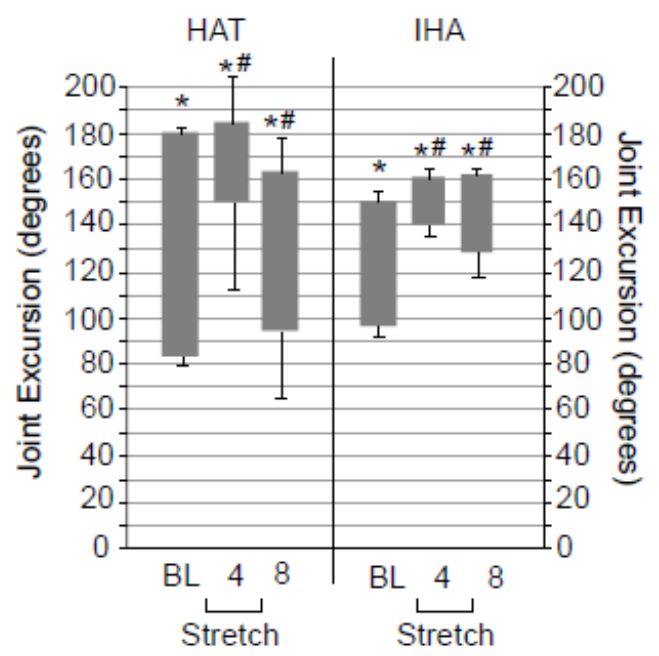

B Chronic Stretch: Joint Kinematics

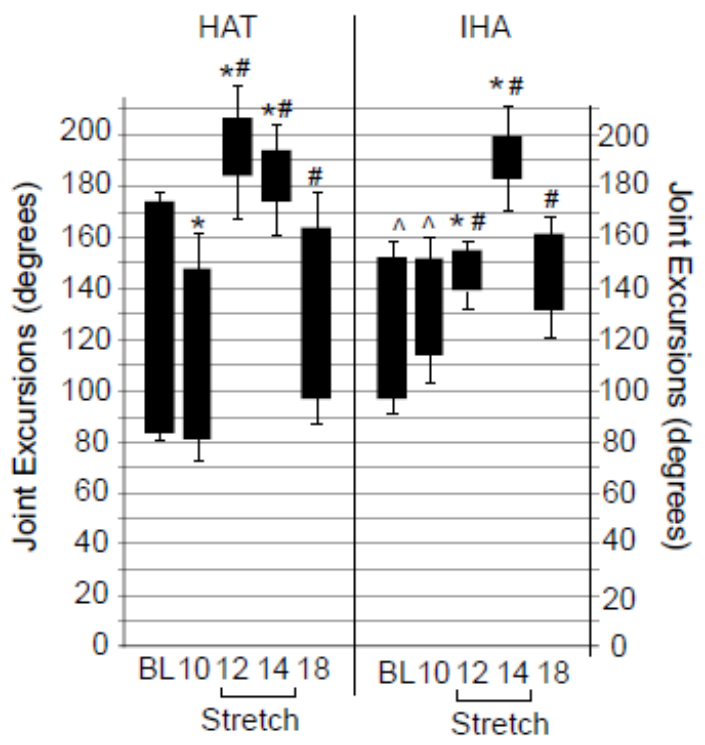

C Acute Stretch: Gait
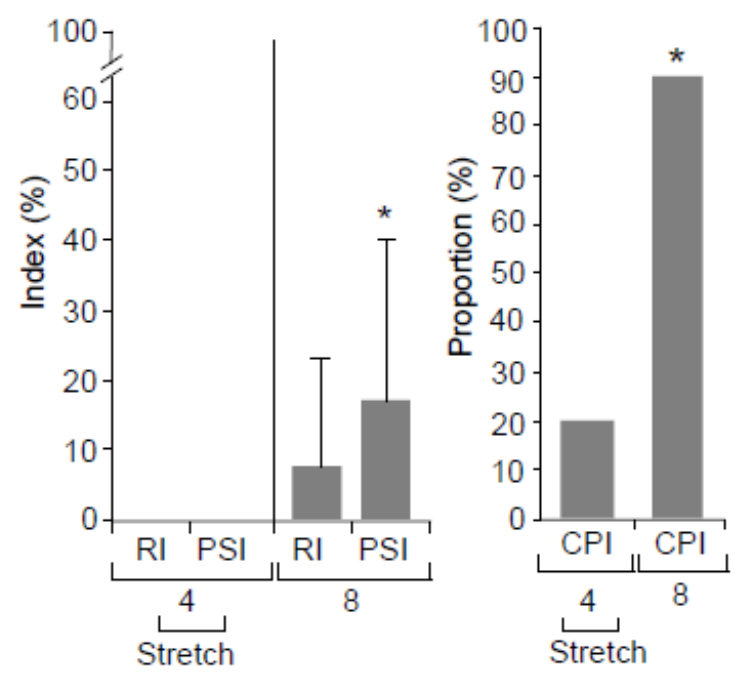

D Chronic Stretch: Gait

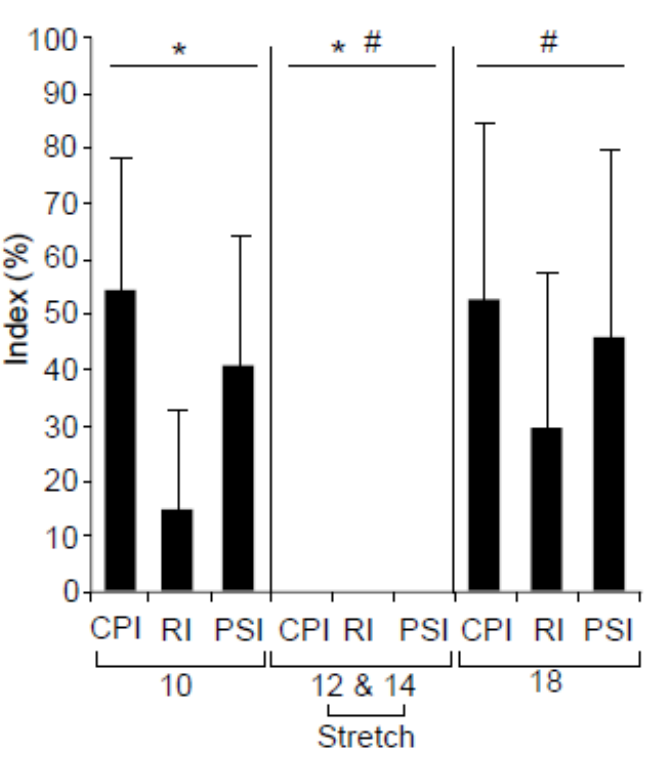


Figure 3. Kinematic and gait analysis of shallow water walking (SWW) of the animals stretched at acute and chronic time points after SCI.

Distal (HAT) and proximal (IHA) joint excursions are indicated by the bars: top and bottom of the bar represents the mean peak extension and flexion, respectively of the joint angles $\pm \mathrm{SD}$ and thus bar length represents the mean angular excursion or ROM. (A) Acute (AcS): Significant differences are indicated by (*) for HAT and IHA joint excursions of AcS animals compared to the ChS group. Significant differences are indicated by (\#) for IHA and HAT excursions of AcS animals at week 4 (during stretching therapy) compared to week 8 (3 weeks after the last stretching session). (B) Chronic Stretch (ChS): Significant differences indicated by $(*$, \#) for ChS animals when comparing weeks 10 or 18 with weeks 12 and 14. In addition, IHA excursions remained significantly lower at week 10 as compared to baseline $\left({ }^{\wedge}\right)$. $(\mathrm{C})$ Acute Stretch $(\mathrm{AcS})$ : Significant differences $(*)$ were seen when comparing CPI, PSI and RI for week 4 and week 8. (D) Significant differences indicated by $(*, \#)$ for $\mathrm{ChS}$ animals when comparing weeks 10 or 18 with weeks 12 and 14. 


\section{Nocturnal In-Cage Activity}

In-cage, overnight activity was monitored and the distances travelled were estimated using a 1 in 10 minutes sampling rate (Figure 4). Even though mixed model RM ANOVA analysis of the AcS overnight distance travelled showed significant differences across time points $(\mathrm{F}=4.9, \mathrm{df}=6,45, \mathrm{p}<.01)$, post-hoc t-tests did not (Figure $4 \mathrm{~A})$. Postinjury distances averaged approximately 150 meters per animal per night by 4 weeks postinjury. Thus stretching did not have an apparent effect on the in cage activity of this group. RM ANOVA showed significant differences in distance traveled of the ChS animals $(\mathrm{F}=7.8, \mathrm{df}=7,48, \mathrm{p}=.001)$. ChS animals averaged $149.3 \mathrm{~m} /$ night at week 8 (prior to stretching intervention) and 67.7 at week 13 (during the third week of stretching), which was significantly lower than week $8(\mathrm{p}<.05)$ while at week $14 \mathrm{ChS}$ group averaged $71.7 \mathrm{~m} /$ night (approaching significance, $\mathrm{p}=.061$ ) (Figure 4B). Furthermore, overnight activity of the $\mathrm{ChS}$ animals was significantly lower $(\mathrm{p}<.05)$ at weeks 12,13 and 14 ( 3 out of the 4 weeks of stretching) as compared to week 20, 4 weeks after the last stretching session. They recovered to $134.7 \mathrm{~m} /$ night by week 20 , which was not different from prestretching (week 8) levels. 


\section{Figure 4}

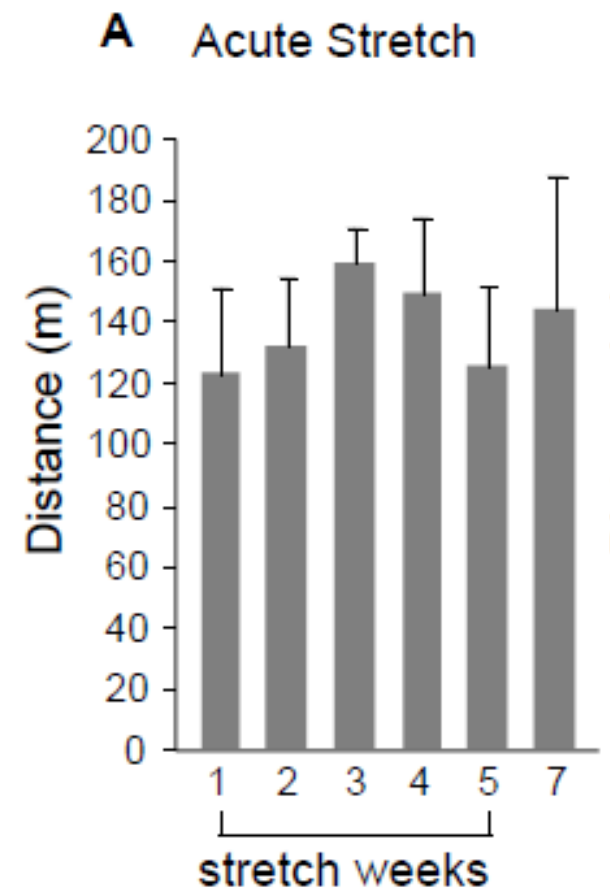

B Chronic Stretch

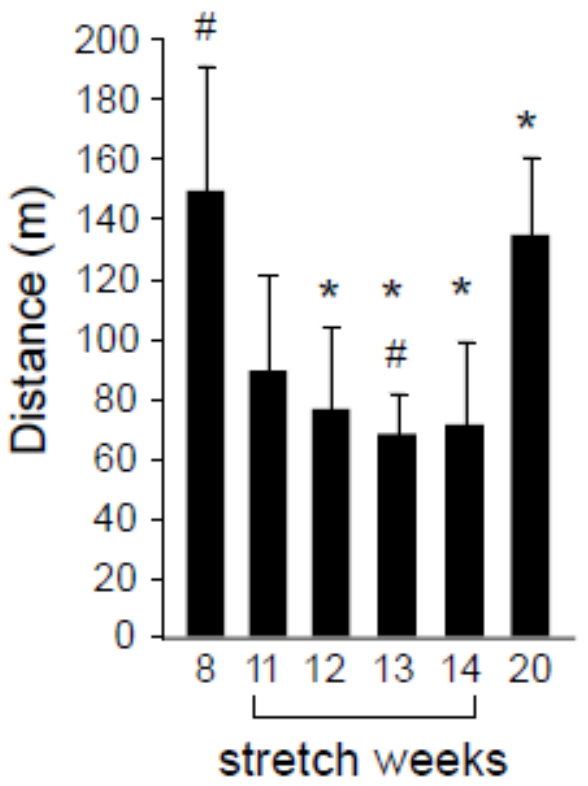

Figure 4. Nocturnal in-cage activity of the animals stretched at acute and chronic time points after SCI.

(A) Acute Stretch (AcS): There were no statistically significant differences in distance travelled in the acute animals at any time points. (B) Chronic Stretch (ChS): Significant differences in overnight activity of $\mathrm{ChS}$ animals are indicated by $\left(\#,{ }^{*}\right)$ for $\mathrm{ChS}$ animals when comparing weeks 8 or 20 with weeks 11-14. 


\section{Magnetically Evoked Muscle Response (MEMR)}

Responses of the gastrocnemius muscle to magnetic stimulation of the base of the tail (MEMRs) were assessed every $4^{\text {th }}$ week of the experiment. These short-latency (56ms), presumed mono- or di-synaptic responses, reflect excitability of the gastrocnemius motoneurons and any changes in the primary afferents and muscles themselves that could influence the EMG signal. Figure 5 shows MEMR data (post-SCI time points normalized to baseline). RM ANOVA showed significant group $(\mathrm{F}=10.8, \mathrm{df}=1,3, \mathrm{p}<.005)$ and time point $(\mathrm{F}=5.16, \mathrm{df}=4,3, \mathrm{p}<.005)$ differences in normalized MEMR. SCI resulted in a significant decrease in EMG amplitude for both acute (AcS) and chronic (ChS) groups (by about 50\%). Stretching had no additional effect on MEMR in the AcS group, as the amplitude of the EMG responses did not differ significantly from the unstretched group $(\mathrm{ChS})$ at week 4. However, the normalized response amplitude of the $\mathrm{ChS}$ animals dropped significantly at week 13 (measured during the $4^{\text {th }}$ week of stretching), a significant decrease from the week 8 (pre-stretch) values. Responses remained significantly decreased at weeks 16 and 20 ( 2 and 5 weeks, respectively, after the last stretching session) even though BBB scores returned to control levels. The onset latency remained unchanged (5-6 ms) throughout in both groups (data not shown). 
Figure 5

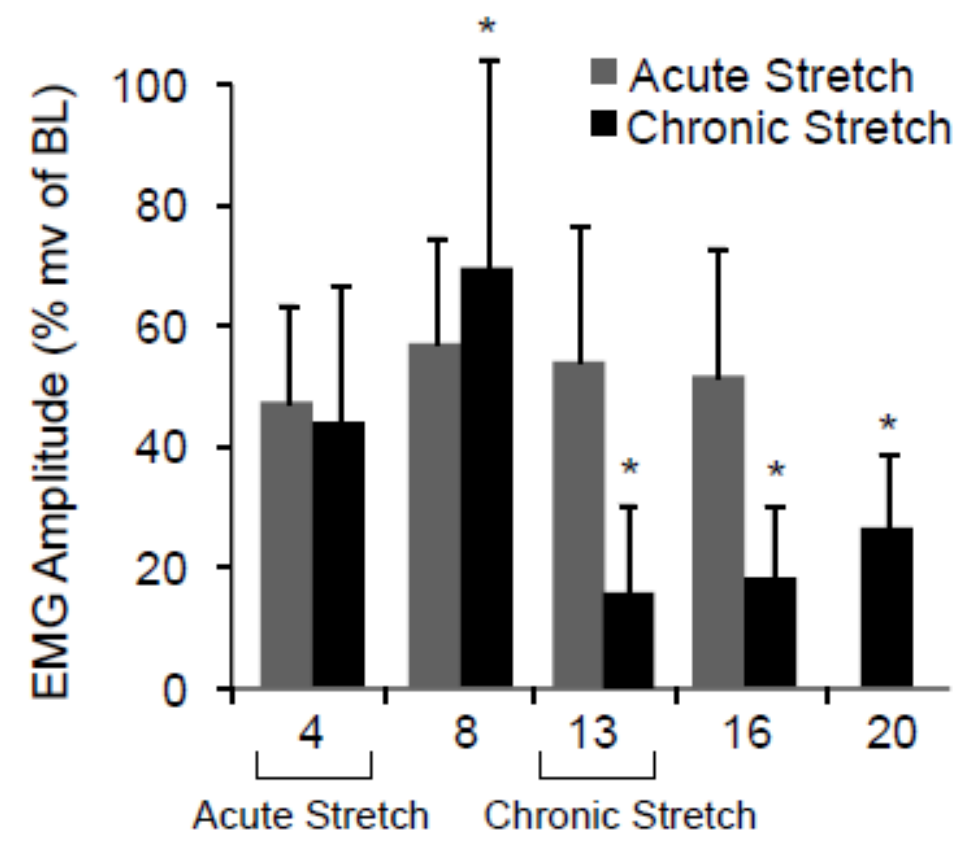

Figure 5. Amplitude of magnetically evoked muscle responses (MEMRs) of the animals stretched at acute and chronic time points after SCI.

Significant differences are indicated by $(*, \#)$ for $\mathrm{AcS}$ and $\mathrm{ChS}$ animals when comparing normalized MEMRs at weeks 13, 16 or 20 to weeks 4 or 8 . There was no difference in MEMRs when comparing AcS and ChS animals at week 4. 


\section{Range-of-Motion}

Significantly reduced locomotor function in the $\mathrm{ChS}$ at the end of the 4 week stretching protocol resulted in the development of contractures around the knee and hip joints. A reduced ROM around those joints was confirmed using a goniometer. RM ANOVA showed significant group differences in the knee $(\mathrm{F}=14.34, \mathrm{df}=1,1, \mathrm{p}<.005)$ and hip $(\mathrm{F}=7.5, \mathrm{df}=1,1, \mathrm{p}<.05) \mathrm{ROM}$ values as well as significant time point difference in hip $\operatorname{ROM}(\mathrm{F}=21.3, \mathrm{df}=1,1, \mathrm{p}<.001)$. At week 15 the ROM around the knee (Figure 6A) and hip (Figure 6B) joints of the $\mathrm{ChS}$ animals were significantly lower compared to control animals $(\mathrm{p}<.01 ; \mathrm{p}<.05$, respectively). At week 18 , when locomotor function of the stretched animals recovered back to pre-stretch levels, the ROM for both previously affected joints had improved significantly $(\mathrm{p}<.01)$ from week 15 and was no longer different from the controls. This suggests that in-cage activity was sufficient to ameliorate the muscle contractures when the animals were not being stretched. 


\section{Figure 6}

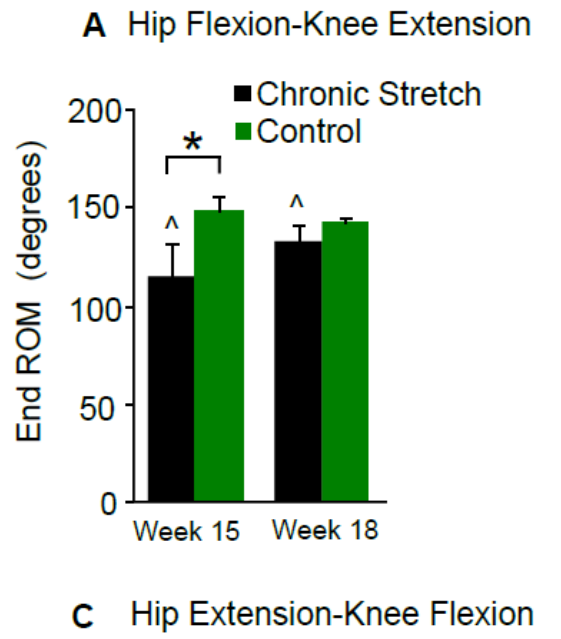

B Hip Flexion-Knee Extension Stick Figure
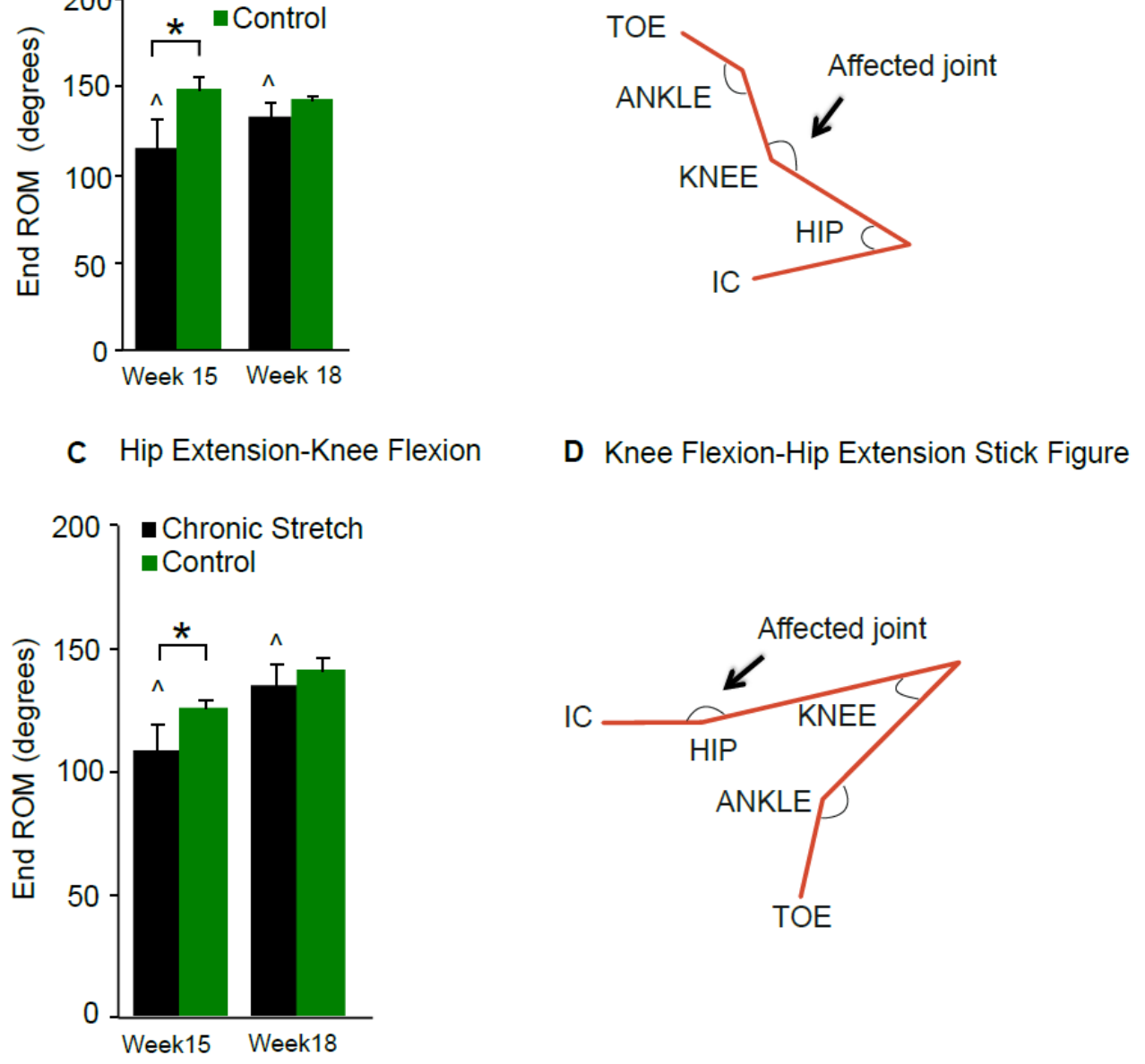

Figure 6. Passive end range of motion of the animals stretched at acute and chronic time points after SCI.

Significant differences are indicated by $(*)$ for knee (A, B) and hip (C, D) angles at end range-of-motion when comparing the $\mathrm{ChS}$ animals with the unstretched control animals at weeks 15 and 18 . By week 18 , the $\mathrm{ChS}$ animals had recovered significantly $\left({ }^{\wedge}\right)$ when compared to week 15 . 


\section{Spinal Cord and Muscle Histology}

There were no significant differences in the percent spared white matter at the injury epicenter, reported as mean $\pm \mathrm{SD}$ : AcS $3.97 \pm 2.33$, ChS $2.64 \pm 1.62$, control $3.77 \pm$ 2.6. Tibialis Anterior (TA), Gastrocnemius (GM) and Biceps Femoris (BF) muscles were analyzed for persistent signs of overt injury induced by repetitive or excessive strain during stretching and that might contribute to the observed locomotor deficits. We also measured cross sectional area (CSA) of muscle fibers to assess if muscle stretch resulted in hypertrophy since it is a potent mechanical stimulus for muscle growth in non-SCI models [183]. The results of the histological analysis are summarized in Table 1. The percentage of muscle fibers that contained centralized nuclei was also not significantly different for any group. We could not identify any areas of fibrosis (tissue scarring) in the three muscles examined and quantitative analysis of collagen revealed no significant differences between the groups. Cross-sectional area of TA, GM and BF muscle fibers in the AcS group were not different from injured, un-stretched controls. One-way ANOVA showed significant group difference in CSA of $\mathrm{GM}(\mathrm{F}=7.5, \mathrm{df}=2,19, \mathrm{p}<.005)$ and $\mathrm{BF}(\mathrm{F}=15.8, \mathrm{df}=2,19$, $\mathrm{p}<.001$ ) indicating that the $\mathrm{ChS}$ group had significantly decreased GM and BF fiber CSA as compared to either the AcS group or to the controls, however no differences in TA fiber CSA were found. These observations suggest that the period of very low activity (weeks 12-15) for the ChS animals resulted in a decrease in muscle fiber CSA (disuse atrophy) in the extensor muscles analyzed. 


\section{Table 1. Skeletal Muscle Histological Analysis}

\begin{tabular}{lccc} 
& \multicolumn{3}{c}{ Muscle Fiber CSA $\left(\mu \mathrm{m}^{2}\right)$} \\
& Tibialis Anterior & Gastrocnemius & Biceps Femoris \\
& & & \\
Acute Stretch $(\mathrm{n}=10)$ & $573.05 \pm 99.00$ & $730.04 \pm 139.75$ & $616.89 \pm 154.77$ \\
Chronic Stretch $(\mathrm{n}=9)$ & $531.52 \pm 116.70$ & $598.83 \pm 122.42^{*}$ & $468.38 \pm 100.84^{\wedge}$ \\
Control $(\mathrm{n}=3)$ & $521.55 \pm 82.26$ & $871.96 \pm 261.74$ & $684.55 \pm 118.28$
\end{tabular}

Centralized Nuclei Count (\% of MF analyzed)

Tibialis Anterior Gastrocnemius Biceps Femoris

$\begin{array}{llll}\text { Acute Stretch }(\mathrm{n}=10) & 3.53 \pm 0.02 & 3.457 \pm 0.02 & 1.669 \pm 0.01 \\ \text { Chronic Stretch }(\mathrm{n}=9) & 2.9 \pm 0.01 & 2.738 \pm 0.01 & 3.294 \pm 0.03 \\ \text { Control }(\mathrm{n}=3) & 2.01 \pm 0.01 & 3.456 \pm 0.01 & 4.283 \pm 0.03\end{array}$

Collagen Area $\left(\mathrm{mm}^{2}\right)$

Tibialis Anterior Gastrocnemius Biceps Femoris

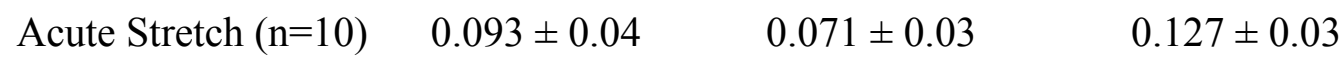

$\begin{array}{llll}\text { Chronic Stretch }(\mathrm{n}=9) & 0.121 \pm 0.5 & 0.073 \pm 0.02 & 0.146 \pm 0.03\end{array}$

$\begin{array}{llll}\text { Control }(n=3) & 0.112 \pm 0.17 & 0.064 \pm 0.01 & 0.116 \pm 0.03\end{array}$

Abbreviations: $\mathrm{CSA}=$ cross sectional area; $\mathrm{MF}=$ muscle fiber

* ChS Gastrocnemius CSA significantly different from Control

$\wedge$ ChS Biceps Femoris CSA significantly different from Control 


\section{Discussion}

These results extend our previous observations of the stretching phenomenon $[165$, 186] into a more clinically relevant moderately-severe animal model of contusive SCI. There are four primary findings in this study. First of all, locomotor function of animals with chronic SCIs is highly vulnerable to the effects of stretching (BBB scores of 0 for all nine animals after the second week of stretching) despite the fact that locomotor recovery of the ChS group had plateaued for more than 4 weeks prior to the initiation of the stretching protocol. Secondly, four or five weeks of stretching did not result in persistent, long-lasting changes in either overground locomotion in the chronic and acute SCI groups as shown by the BBB scores or by shallow water walking as the HAT and IHA "joint" excursions achieved significant recovery. Thirdly, in the chronic group, EMG responses to magnetic stimulation of the base of the tail (MEMRs) remained low for as long as 5 weeks after the last stretching session suggesting a persistent decrease in motoneuron or circuit excitability. Finally, we could find no signs of muscle damage in either AcS or ChS animals that could explain, even in part, the deficits in locomotor function, suggesting that stretching does not result in overt muscle damage that could explain its negative impact on locomotor function and thus neurological mechanisms are likely underlying the locomotor deficits.

Unlike human SCI subjects, animals with only 5-10\% spared white matter achieve significant locomotor recovery [188]. One of the biggest differences between human subjects and rats with SCIs is the state of mobility after the traumatic event. Experimental animals are returned to their home cages (usually with a cage mate) where they move about freely. Flaccid paralysis resolves, in-cage activity increases, and the functional forelimbs provide a built-in training mechanism leading to significant locomotor recovery [188]. On 
the other hand, animals immobilized in wheelchairs after SCI have significant deficits in locomotor function as compared to unrestrained SCI rats [165]. In this study we found that the overnight, in-cage activity (which does not indicate hindlimb involvement in stepping) of the AcS animals remained consistently high throughout the five weeks of stretching, potentially contributing to their quick recovery from the stretch-induced deficits on the weekends. Overnight activity of the $\mathrm{ChS}$ animals, on the other hand, significantly decreased during the weeks of stretching, most likely because these animals used only their forelimbs for propulsion since stretching temporarily negated the hindlimb recovery achieved in the first 10 weeks after SCI. These results support the concept that, hindlimb function associated with higher BBB scores facilitates in-cage distance traveled.

After the last stretching session, animals in both stretching groups recovered significant locomotor function back to control/pre-stretch levels within two weeks. In our previous stretching study the animals also achieved substantial locomotor recovery, however, persistent and significant deficits in function remained [186]. The previous study involved 8 weeks of stretching as opposed to 4 or 5 weeks in the current study. This suggests that longer periods of immobility within the perceived critical window of opportunity for functional locomotor recovery may have resulted in an inability to achieve "full" recovery when returned to standard double-housing. Additionally, animals in the previous study had milder SCIs and higher average BBB scores of 14 as compared to 11 in the current study. Thus, the persistent deficits observed in the previous study may be reflected only in the finer aspects of locomotion, such as hindlimb-forelimb coordination (BBB scores of 11 vs 14) which would not have been discerned in the current study due to the more severe injury and lower functional plateau. 
The focus of physical therapists who employ stretching for SCI patients is to maintain the extensibility of soft tissues and preserve or regain ROM in joints vulnerable to the development of contractures [194]. However, based on a systematic review of stretching for the treatment and prevention of contractures in people with neurological conditions, stretching therapies did not result in clinically important improvements in joint mobility [97]. The authors suggest that a potential limitation in the practice of stretching is a lack of standardized protocols or specific recommendations for the frequency, intensity and duration of stretch to achieve the desired outcomes. One animal study investigated the effectiveness of different stretching characteristics (manipulation of torque intensities and duration) in order to treat the development of knee contractures in animals with complete thoracic spinal cord transections [72]. They found that maximizing both the torque and the duration of stretch resulted in the most significant improvements in contractures (increased ROM). Thus, the authors suggest that high intensity- long duration static stretching may be an effective modality to investigate in clinical trials. However, this study did not consider the potential adverse neurological effects of intense, long-duration stretch. In the present study, when a higher perceived force was required to achieve a normal end ROM during the $4^{\text {th }}$ and $5^{\text {th }}$ weeks of stretching in the AcS group, the negative impact on locomotor function became significant. It appears that the optimal stretching characteristics for treatment and prevention of muscle contractures might also be the most negative for locomotor function after an incomplete contusive spinal cord injury.

Despite the fact that we carefully monitor the hindlimb position for each stretching maneuver and received consultation from experienced human physical therapists, we wanted to investigate if the negative effects of stretching might be partly attributed to overt 
and persistent muscle trauma. Muscle fibers injured by strain undergo robust regeneration via the activation of satellite cells $[195,196]$. Since the nuclei are located peripherally in the myocyte, it is easy to identify fibers that have been injured because regenerating/regenerated muscle fibers have centralized nuclei, which persist for at least 4 months [197]. We found no significant group differences in the number of muscle fibers containing centralized nuclei. Collagen deposition in the extracellular matrix around muscle fibers is another indication of trauma, in particular following repetitive muscle strain [198]. In addition, myocytes that experience persistent or repetitive trauma will eventually fail to regenerate and the debris of dead muscle fibers will be removed and replaced with collagen, resulting in easily recognizable and permanent fibrosis or scarring [195]. Thus, we quantified collagen in the muscles but again found no differences between the three groups. We therefore concluded that our stretching protocol does not result in frank and persistent muscle damage that can help explain the significant locomotor deficits that we observe in our animals.

The severe locomotor dysfuction, significantly reduced in-cage activity and contractures (reduced passive end ROM of the knee and hip) in the ChS rats was accompanied by significant decreases in the CSA of GM (ankle extensor) and BF (knee flexor/hip extensor) muscles, suggesting disuse atrophy. However, the Tibialis Anterior (ankle flexor) muscle did not undergo significant atrophy. Previous studies found a similar pattern of muscle atrophy as a result of disuse affecting extensor muscles more than flexors $[199,200]$.

It is worth considering, however, that muscle stretch does not affect only the muscles, tendons and ligaments. Stretching will also affect group Ia and II muscle spindle 
afferents, potentially increasing their threshold for firing (decreasing their sensitivity to stretch). Given their importance for the generation/recovery of locomotion after SCI [121], changes to muscle spindle sensitivity is one potential explanation for the stretching phenomenon. In addition to Ia and group II muscle spindle afferents, muscles are innervated by small diameter thinly myelinated and unmyelinated group III and IV afferents that are activated by mechanical stimuli such as contraction and muscle stretch [201-204]. Specifically, Cleland et al, have identified stretch-sensitive free nerve endings of group III and IV fibers that mediate powerful reflex inhibition of the homonymous muscles in an animal model of clasp-knife reflex [203]. This same group has also found force-sensitive interneurons that receive input from group III and IV afferents and that produce rapid and sustained inhibition of motoneuron output [205]. Group III and IV afferents have also been implicated in the inhibition of motor output (central fatigue) during exercise in humans $[206,207]$. Thus, it is probable that the stretching protocol we administer to spinal cord injured animals activates group III and IV afferents which in turn should have an inhibitory affect on motor circuitry resulting in dramatic drops in locomotor function. Whether or not this mechanism is responsible, in whole or in part, for our current observations remains to be determined.

After an incomplete spinal cord injury in rats, the sprouting of spared descending axons is thought to mediate at least some of the remarkable functional recovery [208]. On the other hand, primary afferent sprouting, particularly of $\mathrm{C}$ and $\mathrm{A} \delta$ fibers has been associated with neuropathic pain and autonomic dysreflexia [209, 210]. It is possible that increased arborization of these primary afferents also leads to the more robust inhibitory effects of group III and IV afferents over motor output thus explaining why animals with 
stabilized locomotor recovery (ChS group) are so vulnerable to the negative effects of stretching. If this hypothesis is confirmed, it is likely that the stretching phenomenon we observe in our rats has high clinical relevance. Harvey et al, investigated the amount of torque physical therapists apply during regular stretching sessions and found that some therapists applied torques that were 2 to 6 times higher than what is tolerated by sensate individuals. In addition, the authors discuss the possibility that SCI patients regularly generate very high torques around their hip joints while dressing in a seated position [211]. For the majority of SCI patients, stretching that activates nociceptive afferents would not result in the perception of pain due to the loss of sensory function below the level of injury. However, existing evidence suggests that transmission of nociceptive signals results in a multitude of unfavorable effects over the locomotor circuitry $[212,213]$ that has an otherwise high capacity for retraining, given the appropriate proprioceptive feedback [153, 214-216].

In conclusion, stretching has been adopted as a therapy in the rehabilitation regime for SCI patients based in part on past evidence from animal studies that focused on soft tissues undergoing maladaptive changes as a result of immobilization [73]. While more recent clinical investigations into stretching therapy reveals its general ineffectiveness for the treatment of muscle contractures in SCI patients $[97,181]$ current and previous findings from our laboratory suggest that stretching is detrimental to locomotor function in animals with mild to severe SCIs at both acute and chronic time points. The clinical relevance of our results is yet to be determined, but these findings suggest strongly that the neurological effects of muscle stretch warrants consideration as being potentially detrimental to the function of locomotor circuitry following spinal cord injury. 


\section{CHAPTER IV \\ DYNAMIC “RANGE OF MOTION” HINDLIMB STRETCHING DISRUPTS \\ LOCOMOTOR FUNCTION IN RATS WITH MODERATE SUBACUTE SPINAL \\ CORD INJURIES}

\section{Introduction}

Spinal cord injury (SCI) that causes paralysis below the level of the lesion results in a multitude of secondary complications that commonly include joint contractures and spasticity. [217] Both complications can significantly reduce the quality of life of SCI patients. [47] Currently, stretching remains the first line therapy to treat and prevent contractures and spasticity. $[179,181,182]$ Previously in our laboratory we showed that a 24 minute protocol of "static" stretching of the major hindlimb muscles of rats with mild to moderately-severe acute and chronic SCI results in significant declines in locomotor function. [171, 218] The effects of therapeutic stretching on motor function of SCI patients is currently not known; the main outcome measures of clinical studies on stretching are range of motion and/or spasticity. [194] However, a multitude of studies have investigated static stretching in uninjured athletic populations and have observed negative effects on some aspects of motor function. [219] Specifically, static stretching results in decrements in vertical jump and running performance, [220-222] muscle strength endurance, [223] isometric strength [224, 225] as well as isokinetic torque production. [226, 227] Dynamic stretching is an alternative technique to improve range of motion (ROM) and flexibility that has been recommended as part of the warm up routine for athletic events; it is not 
thought to cause detriments in performance and may actually improve it. [228, 229] Dynamic stretching involves multiple repetitions of limb movement through its entire range to the end ROM. [77] Such range of motion exercises are also commonly performed during the rehabilitation of SCI patients. [230] Therefore, we wanted to determine if a dynamic stretching protocol involving all major hindlimb muscle groups in rats with SCI disrupts locomotor function similarly to our static stretching protocol. For a variety of reasons including a reduction in the total time at end ROM and the involvement of a rhythm, we hypothesized that dynamic stretching would not be detrimental to locomotor function in rats with moderate spinal cord injuries.

\section{Materials and Methods}

Eight young adult female Sprague-Dawley rats were used in this experiment. All procedures were approved by the Institutional Animal Care and Use Committee at the University of Louisville. After a week of acclimatization and baseline data collection, the animals received moderate spinal cord contusion injuries (NYU, $12.5 \mathrm{~g} / \mathrm{cm}$ ) at T10 as described previously. [191] Locomotor function was assessed at least weekly using the BBB Open Field Locomotor Scale [187] and video-based kinematic and gait analysis was done biweekly. As described previously,[218] we used a 3 segment, 2 angle model of the hindlimb and "joint" excursions were measured as the hip-ankle-toe (HAT) and iliac cresthip-ankle (IHA) angles. Two gait indices were also used, the regularity index (RI) and the plantar stepping index (PSI) based on ventral-view video allowing paw placement order, timing and location to be determined for every step taken (from initial contact to lift-off) using MaxTraq software (Innovision Systems Inc., Columbiaville, MI) and custom designed Excel macros. The regularity index (RI) is calculated as the number of correctly patterned plantar steps over the total number of steps (dorsal and plantar). [231] The plantar 
stepping index (PSI) is calculated as the number of plantar hindlimb steps over the total number of forelimb step.[188]

At six weeks post injury the animals entered a four week dynamic stretch protocol that was performed 5 days a week for 4 weeks. Dynamic stretching consisted of the same six bilateral stretches of major hindlimb muscle groups as our previous static stretching protocol, $[171,218]$ however, instead of holding the limb at the end ROM of each stretch for $1 \mathrm{~min}$, each stretch was held for only 2 seconds, followed by a 1 second release, repeated 20 times over a one minute period. A metronome (at $1 \mathrm{~Hz}$ ) was set to the $2-1$ ratio, using different tones, in order to give an audible clue to the rat physical therapists (PT) allowing them to maintain the rhythm and ensuring that timing was consistent and accurate between therapists for each animal every day. During the 4 weeks of stretching, BBB scores were assessed three times weekly (Monday am, Monday pm and Friday pm).

Tonic stretching triggers several observable responses in both the stretched and unstretched limbs that rat PTs take note of [171]. Dynamic stretching resulted in a new and highly consistent response that was observed as paw "vibrations": high frequency and low amplitude fluctuations in one or two joints. Because of its resemblance to human clonus, which can be triggered by sudden stretching or upon the release of stretch, [232, 233] we sought to better understand this phenomenon. In order to quantify this response, single stretching sessions for four animals were recorded (digital video at $100 \mathrm{~Hz}$ frame rate with the same PT stretching all animals). Most often vibrations occurred in both limbs simultaneously, however, kinematic analysis was performed on the contralateral limb as more joint markers were easily viewable and could be tracked with the software for analysis. The toe, ankle, knee, hip and iliac crest were marked with a black marker for 
kinematic analysis of the movement using MaxTraq software. Three vibration responses per stretch per animal were analyzed for range of motion (excursion of movement) and frequency (peaks per second). Averages of these outcome measures from the four animals for each stretch are reported in the results.

After the last week of stretching (week 4), four of the eight animals were sacrificed 2 hours after the last stretching session and the other four animals were maintained for an additional 5 weeks. The spinal cords and major hindlimb muscles (Tibialis Anterior (TA), Medial Gastrocnemius (MG) and Biceps Femoris (BF) were dissected out, post-fixed in $4 \%$ PFA and cryoprotected in 30\% sucrose. Muscles (mid-belly) were sectioned at $10 \mu \mathrm{m}$ and stained with Hematoxylin and Eosin. Muscle fibers (MF) were analyzed for the presence of centralized nuclei, a marker of regeneration. [195] Spinal cords were sectioned at $50 \mu \mathrm{m}$ and stained with eriochrome cyanine to determine the percentage of white matter sparing at the injury epicenter using ImageJ software as described previously. [191]

Each animal served as its own control for the locomotor outcome measures and the data was analyzed for changes over time using a mixed model analysis of variance (ANOVA; BBB scores and joint excursions) followed by Tukey post hoc. Non-parametric Wilcoxon Signed Ranks test was used to make comparisons between time points in the gait indices RI and PSI. One-way ANOVA followed by independent T-tests were used to compare the percentages of MF containing centralized nuclei between the animals sacrificed 2 hours after the last stretching session (group 1, $\mathrm{n}=4$ ) and the animals sacrificed 5 weeks later (group 2, $\mathrm{n}=4$ ). Significant differences were established at $\mathrm{p}<.05$.

\section{Results}

Based on a dense, compact appearance on eriochrome-cyanin stained sections, moderate SCI at T10 resulted in an average spared white matter (SWM) at the injury 
epicenter of $7.35 \% \pm 3.36$. The average SWM for group 1 was $6.08 \pm 2.54$ percent and 8.61 \pm 3.96 percent for group 2 . There was no significant difference in SWM between the groups sacrificed at two different time points $(\mathrm{p}=.322)$. Despite the low percentage of spared white matter the animals achieved substantial locomotor recovery: BBBs plateaued by week 6 with an average score of 11, which is indicative of consistent weight supported stepping without forelimb-hindlimb coordination (Fig.1). One week of dynamic stretching starting at 6 weeks post-SCI resulted in a significant decrease in locomotor function as average BBB scores dropped to $3.7 \pm 2.3(\mathrm{p}=.004)$. Thereafter, at the end of each week of stretching BBB scores hovered around 2.5 (indicative of slight movement of one or two joints only). As in previous studies, these animals achieved some locomotor recovery over the weekends when they were not stretched. [186, 218] However, one stretching session (Monday morning) was enough to again reduce the BBB scores to below 4 (Fig.1). Lack of hindlimb movement was further confirmed with the more objective kinematic and gait analysis. The animals had significantly reduced excursions (range of motion) of both IHA and HAT hindlimb angles during the weeks of stretching (W6 and W8). RI and PSI were reduced to 0 at week 6 (measured on the third day of the first week of stretching) indicating a complete lack of plantar stepping (Fig.2B). These gait indices remained at 0 until stretching ceased. Figure 2 depicts some characteristics of the vibration response. In B, a stick figure is shown representing a single episode of the vibration response upon the release of stretch (in this case of the hamstring stretch): black line, a starting position and gray lines showing a full range of motion the limb goes through during the response. As the foot "vibrates" (multiple flexion/extension phases) the positions of the toe, ankle and knee change, and A shows the velocity profile of these points. Table 1 shows the average 
frequencies (calculated as number of peaks in knee-ankle-toe (KAT) per second ) and averaged ROM (degrees) in the KAT angle during the vibration response for each stretch. The percentage of muscle fibers containing centralized nuclei for each muscle analyzed are listed in Table 2. Animals sacrificed 2 hours after stretching had a significantly higher percentage of muscle fibers with $\mathrm{CN}$ in the MG muscle as compared to the group sacrificed 5 weeks later $(\mathrm{p}<.05)$. There were no significant differences for the BF and TA muscles. 


\section{Figure 7}

A. BBB Open Field Locomotor Scores

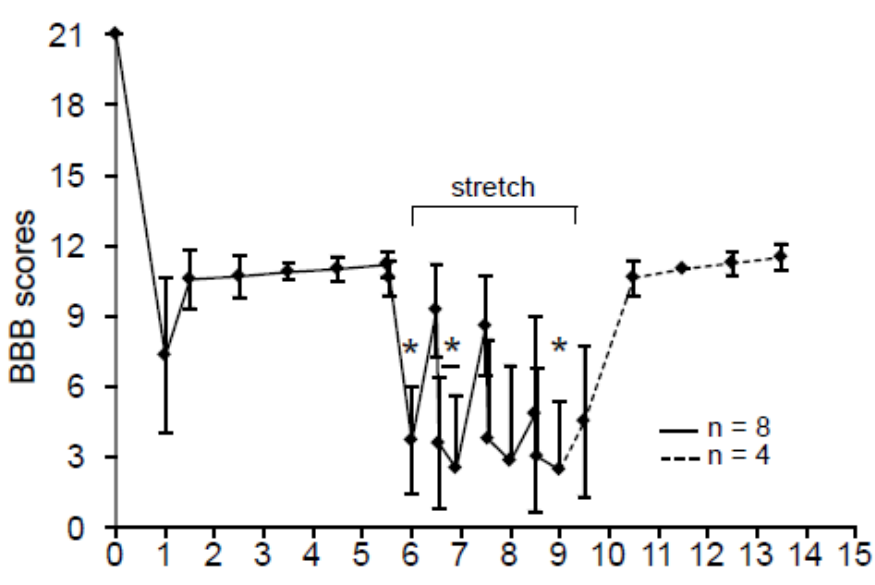

Weeks post-SCI

B. 3D Kinematics: Joint Excursion

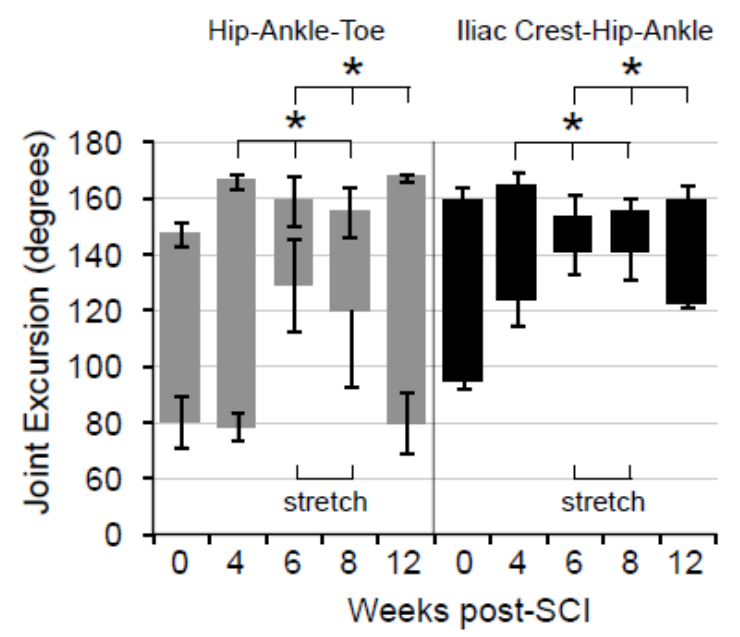

C. Gait Indicies

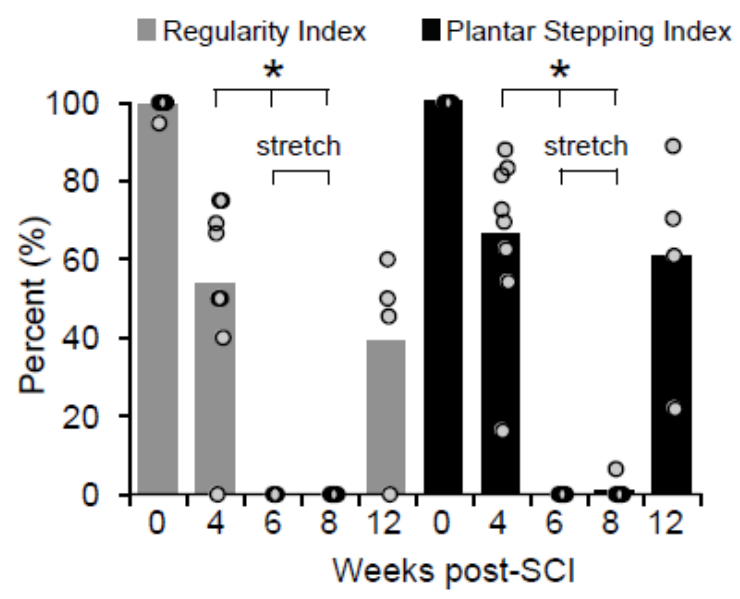


Figure 7. Locomotor function of the animals with sub-acute SCI in response to dynamic stretching protocol.

A. Dynamic stretching protocol began 6 weeks post-SCI when locomotor function has reached a stable plateau and continued on for 4 weeks. At the end of the first week of stretching, the animals had significantly lower BBB scores as compared to their pre-stretch values. Over the weekend animals achieve significant recovery, however, the first session of the second week of stretching induced deficits in the locomotor function that persisted to the end of the week. Similar pattern of recovery/disruption followed for the remaining two weeks of the stretching protocol. Four of the eight animals were sacrificed after two hours of stretching for histological muscle assessment and the other four were allowed to recover for 4 weeks before being sacrificed. Their locomotor function was assessed weekly (dotted line). B,C. Analysis of biweekly kinematic and gait recordings showed that the animals had significantly reduced joint excursions (B) and gait indices (C)) indicative of lack of stepping or even sweeping in some animals during the weeks of the stretching therapy (week 6 and 8) as compared to the pre-stretch stepping ability and hindlimb joints excursions (week 4). By week 12 the locomotor function of the remaining 4 animals had achieved significant recovery back to pre-stretch levels. 


\section{Figure 8}

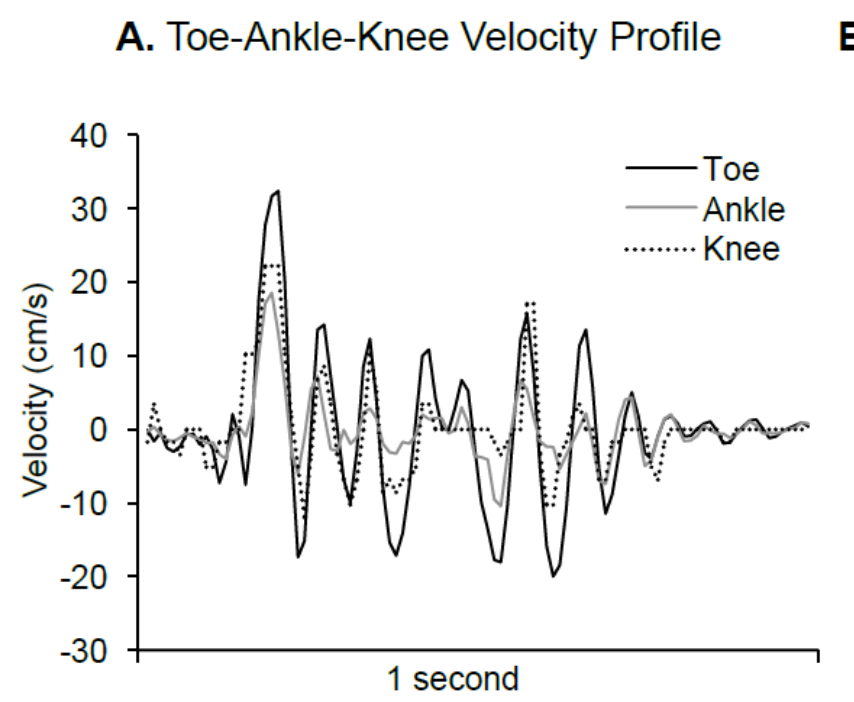

B. Stick Figure of Vibration Response

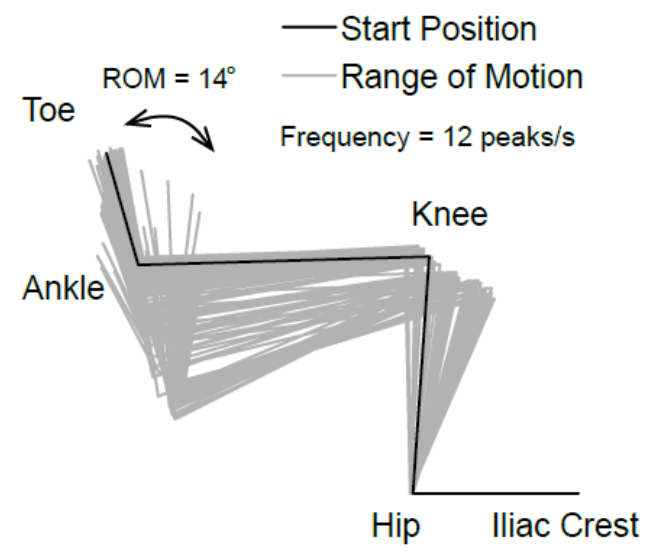

Figure 8. Characteristics of the vibration response to the dynamic stretching protocol in rats with sub-acute SCI.

Kinematic analysis was performed on the contralateral limb vibration response during a release of 2 second hamstring stretch of the opposite limb. A. During a vibration response the limb goes through multiple clonic-like contractions as evidenced by the multiple peaks in velocity of the toe-ankle-knee angle in one second. B. Stick figure of the contralateral limb during a vibration response. The black line shows a steady position of the limb during the 2 second stretch hold period of the opposite limb. Upon release of stretch, the cloniclike contractions (gray stick figures) are initiated as the limb is pulled in towards the body (knee and hip are flexed). Most of the movement occurs in the ankle joint with the average range of motion of 14 degrees and contractions occurring at high frequency (about 12 peaks per second). 


\section{Discussion}

In this study we sought to determine if an alternative dynamic hindlimb stretching protocol would result in disrupted locomotor function in rats with SCI. Based on the simple concept that phasic or rhythmic afferent input would be less detrimental to spinal cord circuitry function as it relates to locomotor function than tonic afferent input, [150] we hypothesized that dynamic stretching would not induce locomotor deficits. Contrary to our hypothesis, dynamic stretching dramatically disrupted locomotor function reducing BBB scores to levels similar to those of static stretching even though the total amount of stretch that the animals received on a daily basis was less (20 seconds less/stretch) than in our previous studies. [186, 218] Histological analysis of the muscles revealed that for the ankle extensor MG the group sacrificed two hours after stretching had elevated numbers of centralized nuclei $(8 \%)$ as compared to the rats sacrificed four weeks later $(\sim 4 \%)$ suggesting that more muscle fiber regeneration was occurring due to the strain resulting from multiple stretching sessions. It is important to note however, that the additional muscle fiber regeneration was apparently complete by 4 weeks suggesting that the stretching did not cause damage beyond what the muscle repair system is equipped to handle and is thus unable to explain the locomotor deficits. Nevertheless, this is the first evidence suggesting some degree of muscle damage due to stretching, potentially because our previous studies had animals surviving for several weeks after the last stretching session.

During stretching, our rat physical therapists keep a thorough record of all observable hindlimb responses amongst which are contralateral limb kicking, air stepping and spasms (contralateral and ipsilateral limbs). A new response was uncovered with dynamic stretching that we refer to as "vibrations". These were induced by the onset and 
the release of stretch and were seen in the both the stretched and the contralateral unstretched limb. Although we did not record electromyography (EMG) during these responses we did determine the kinematics of the vibrations. We found that the vibrations had a frequency range of $10-19 \mathrm{~Hz}$ with a mean of 14.42 for all stretches combined, and a very low amplitude around 10 degrees (in the KAT angle). Onset of muscle contractions when the limb is being stretched and the muscles lengthened, or in other words, eccentric muscle contractions, are being generated during our stretching protocol. Eccentric muscle contractions occur at the ground contact phases of normal locomotion are a part of stretchshortening cycle, one of the fundamental functions of the skeletal muscle. [234] This type of muscular activation serves to decelerate the limb for smooth and controlled contact with a surface [235] and is often utilized in athletic training because it provides an excellent stimulus for muscle hypertrophy and strength gains. [236-238] However, eccentric contractions have been also recognized to result in a greater amount of disruption in myofibrillar structure [239] which could potentially explain why we see an increased number of regenerating fibers in the muscles of the animals that were sacrificed within hours of the last stretching session. Delayed onset muscle soreness (DOMS) which is a type of mechanical hyperalgesia, occurs 1 to 2 days after eccentric contractions as a result of nociceptive afferents sensitization in response to release of bradykinin and nerve growth factor. [240] Consistent with the DOMS phenomenon is the following observation: we have never observed any decrements in locomotor function following the initial stretching session in this (Fig. 1) or any of our earlier studies. [218] 
Table 2. Characteristics of clonic-like muscle responses to stretch.

Muscle/Stretch

Tibialis Anterior

Gastrocnemius

Quadriceps

Hamstring

Hip Abductors

Hip Adductors
Clonic-like Stretch Response Characteristic Frequency (peaks/s) Range of Motion (degrees)
$12.86 \pm 3.44$
$16.24 \pm 12.29$
$13.29 \pm 5.55$
$11.74 \pm 7.81$
$16.35 \pm 1.41$
$7.04 \pm 2.29$
$15.38 \pm 1.72$
$8.56 \pm 3.55$
$15.48 \pm 2.65$
$8.33 \pm 8.28$
$13.14 \pm 2.51$
$2.96 \pm 0.93$

Data reported as means $\pm \mathrm{SD}$

Table 3. Percent of muscle fibers with centralized nuclei.

Muscle

Tibialis Anterior

Medial Gastrocnemius

Biceps Femoris
Percent of muscle fibers with centralized nuclei.

$\begin{array}{ll}\text { Group } 1(\mathrm{n}=4) & \text { Group } 2(\mathrm{n}=4) \\ 3.77 \% \pm 1.62 & 4.7 \% \pm 1.81 \\ 8.21 \% \pm 2.06^{*} & 4.18 \% \pm 1.12 \\ 4.49 \% \pm 2.45 & 4.65 \% \pm 1.53\end{array}$

Data reported as means $\pm \mathrm{SD}$.

Group 1 - animals sacrificed 2 hours after the last stretching session; Group 2 - animals sacrificed 5 weeks after the last stretching session;

*Group 1 had significantly greater percentage of muscle fibers with centralized nuclei in the Medial Gastrocnemius muscle as compared to Group $2(\mathrm{p}<.05)$. 
However, in the second week of stretching, one session does cause the deficits, presumably due to the sensitization of afferents.

Nociceptive afferents have complex modulatory effects on locomotor circuitry. Kniffki et al., have observed that stimulation of group III and IV afferents can either accentuate an ongoing fictive locomotor rhythm in spinal cat preparation or block the preexisting rhythm by inducing a period of tonic hyperactivity [241]. It has been also established that nociceptive afferents converge on common interneurons in the reflex pathways to alpha motoneurons and have both facilitatory and inhibitory effects on transmission of other reflex afferents that modulate locomotor function [242]. Functionally, nociceptive facilitation of efferent response to a potentially damaging stimulus is readily seen in withdrawal reflex. On the other hand, activation of group III and IV afferents during exercise has been shown to reduce motor output, a phenomenon known as central fatigue [206]. Furthermore, sensitization of these afferents during DOMS may contribute to the impairments in maximal force generation and rate of force development [243-245]. In healthy humans activation of nociceptive afferents may only have mild inhibitory effects on locomotor function as supraspinal modulation may limit their activity. It has been shown that electrical stimulation of mesencephalic locomotor region in the cat inhibits group III and IV transmission in the dorsal horn [246]. After SCI, however, central pattern generators in the lumbar cord become much more reliant on peripheral inputs for their activation[121] and therefore locomotor circuitry is also likely more vulnerable to inhibitory effects of group III and IV afferents. Moreover, nociceptive afferents undergo substantial plasticity after SCI that has been implicated in the development of neuropathic pain [247, 248] and autonomic dysreflexia[249]. Activation of nociceptive afferents 
impairs locomotor recovery after SCI in rodents and spinal learning in rats [212, 213, 250] and humans [251]. Thus it would not be surprising that nociceptive afferents are also involved in mediation of the negative effects of stretching on locomotor function.

In conclusion, muscle stretching is a commonly used technique aimed at increasing/maintaining joint ROM and is used across multiple populations from athletic to clinical. We have now shown that both static and dynamic stretching applied to the hindlimbs of spinal cord injured animals result in the dramatic but temporary disruption of their locomotor function. The physiological mechanism of this phenomenon is not yet known, although based on our observations and histological findings it is unlikely due to frank muscle damage, but may involve activation of nociceptive afferents. The clinical relevance of our findings needs to be established but given that static stretching has been shown to have detrimental effects on performance in healthy humans $[219,224,252]$ it is conceivable that after SCI the motor circuitry is even more vulnerable to the negative effects of stretch. 


\section{CHAPTER V \\ ELECTROMYOGRAPHIC PATTERNS OF THE RAT HINDLIMB IN RESPONSE TO MUSCLE STRETCH AFTER SPINAL CORD INJURY}

\section{Introduction}

Stretching remains a leading therapy for the treatment of muscle contractures as well as spasticity, two of the most common complications after a severe spinal cord injury (SCI). Moreover, stretching is a rehabilitation strategy often employed acutely post-SCI in an effort to prevent the development of muscle contractures because they are much harder to treat once established [73]. The rationale for using stretching in the rehabilitation of soft tissue contractures arose initially from animal studies that showed stretching to be effective at maintaining joint range of motion (ROM) due to immobilization [68]. However, stretching for the treatment or prevention of contractures in SCI patients does not have the same efficacy [97]. We determined recently that a daily protocol involving either static or dynamic application of stretch, disrupts hindlimb locomotor function in rats with SCI [218]. The mechanisms underlying the stretch-induced loss of locomotor function are not known but based on our previous findings they do not involve overt muscle damage [218]. It is well documented that in healthy subjects static stretching decreases strength and maximum performance $[220,223,225,227]$. Avela et al., have shown that repeated and prolonged stretching results in decreased EMG amplitude during maximum voluntary 
contraction, suggesting a central mechanism [224]. Interestingly, Cramer et al., observed strength loss not only in the stretched limb but also in the contralateral non-stretched limb, further implicating a circuitry-based mechanism [253]. Given these observations and widespread use of stretching in the rehabilitation/physical therapy of patients with SCI it is important to establish if the negative effects of stretching on locomotor function in rats with SCI are clinically relevant. Therefore, we designed and performed an experiment using tools that would allow direct comparison with human studies, such as electromyography, force and torque measurements and limb kinematics during stretching. We hypothesize that EMG patterns represent the response of the spinal cord to the afferent input generated by stretching and thus can serve as a translational cue: if responses are similar for rats and humans, then it is likely that the effect of stretching on motor circuitry are also similar. Force measurements will provide context for the EMG responses and will help set the parameters for future clinical studies on stretching after SCI.

\section{Materials and Methods}

Animals, EMG transmitter instrumentation and spinal cord injury.

Four adult Sprague Dawley rats were used for this experiment. All procedures were approved by the Institutional Animal Care and Use Committee at the University of Louisville. After 1 week of standard gentling procedures and baseline assessments the animals underwent an initial surgery for instrumentation with a telemetry-based 2 lead EMG transmitter (F20-EET, Data Sciences International ${ }^{\circledR}$, St. Paul, MN;). The animals were anesthetized with a ketamine cocktail and a surgical level of anesthesia was confirmed by the absence of paw withdrawal reflexes to strong foot pinch. The upper back of each animal was shaved and cleaned and a small skin incision was made between the shoulder blades where the body of the transmitter was placed. The wires of the transmitter were 
tunneled subcutaneously down to the right hindlimb by separating the skin from the muscle layer using blunt dissection. A small incision was made over the thigh of the animal allowing the wire electrodes to be inserted into and through the belly portion of the Rectus Femoris and Biceps Femoris muscles. Sutures were placed on each side of the wire to hold it in place. The incisions were closed with sutures and animals were allowed to recover, receiving our standard post-operative care with antibiotics (gentamicin sulfate for 7 days $(20 \mathrm{mg} / \mathrm{kg}))$ and pain management (buprenorphine $0.03 \mathrm{mg} / \mathrm{kg}$ twice a day for 3 days). Two weeks later the animals underwent a second surgery and received a mild-moderate spinal cord contusion injury at thoracic level T10 $(12.5 \mathrm{~g} / \mathrm{cm}, \mathrm{NYU}$ contusion device $)$ as previously described [185]. The animals were then allowed to recover for two weeks prior to the beginning of stretching.

Stretching and recording procedures.

In this study we used 6 stretches (1 minute static hold) of the major hindlimb muscle groups from our previously described stretching protocol [171]. For the first 6 weeks the stretching protocol was carried out twice a week (Monday and Wednesday) only on the left (non-implanted) limb, while recording the responses of the contralateral (implanted) limb. For weeks 7 and 8 we switched to daily stretching and included two ankle stretches (gastrocnemius and tibialis anterior) of the implanted limb. For the final stretching and recording session (week 8 Friday) all 6 stretches were performed on the implanted limb. EMG data was acquired through the DataQuest Acquisition hardware (Data Sciences ${ }^{\circledR}$ International, St. Paul, MN) and PONEMAH® 5.0 software package (sampled at $1000 \mathrm{~Hz}$ ). The data was exported for further analysis to LabChart (ADInstuments Colorado Springs, CO). Within the DSI system, the receiver and the transmitter communicate at a set 
frequency of $455 \mathrm{kHz}$. This reduces the possibility of signal contamination with nonspecific frequencies from surrounding electronics. During recording, the animals were placed directly onto the receiver ensuring a strong signal for data transfer from the transmitter to the receiver. These features, in addition to the isolation of the preamplifier, transmitter and the EMG leads within the animal ensure a high quality EMG signal with a very high signal to noise ratio and with no noticeable $60 \mathrm{~Hz}$ contamination. The raw EMG data was exported for further analysis to LabChart (ADInstuments Colorado Springs, CO). The raw EMG signal was band-pass filtered at $50-2000 \mathrm{~Hz}$ to remove movement artifacts. The analysis of clonic-like responses for amplitude and frequency (in one second) was performed on rectified and smoothed (average of 101 data points) EMG using LabChart's peak analysis feature. Videos of the implanted limb were linked to EMG recordings and each analyzed clonic-like response was confirmed visually in the video.

\section{Forces applied during stretching.}

The forces applied during stretching were measured using a custom designed force glove (FlexiForce A301 sensors connected to a USB-6210 data acquisition system) operated by LabView software. Force sensors were calibrated to standardized weights of 105,305 and 505 grams prior to each stretching session. Force sensor data was sampled at $1000 \mathrm{~Hz}$. Sensors for the thumb (TS) and index finger (IS) were used to measure forces during the four stretches. They were placed as follows: for stretch of the Tibialis Anterior (ankle flexor) TS was placed on the dorsal portion of the foot and IS on the heel; for stretch of Triceps Surae we used only one sensor (TS), placed on the mid plantar portion of the foot; for quadriceps TS was placed on top of the knee (which was bent during this stretch), while IS was placed at the mid dorsal portion of the foot; and for the hamstring stretch TS 
was placed at the heel and IS - on the thigh slightly superior to the knee which was extended for this stretch. The forces were not measured during hip abduction and adduction stretches due to difficulty of locating precise points of force application. The positions of the toe, ankle, knee, hip and iliac crest, along with the sensors were digitized using MaxTraq software (Innovision Systems, Inc., Columbiaville, MI) to determine the distances between the joints and sensors allowing torque to be calculated (torque $=$ force*distance $(\mathrm{cm}) * 9.8$ $\left(\mathrm{m} / \mathrm{s}^{2}\right) / 1000(\mathrm{~g} / \mathrm{kg})$, where 9.8 is the gravitational acceleration constant).

\section{Locomotor function assessments.}

Locomotor function was assessed using 3D kinematics and gait analysis based on paw placement order. Horizontal and ventral view recordings were made pre- and poststretching for each session. As previously described [185, 188] we used a 3 segment 2 angle model of the hindlimb for kinematics - iliac crest-hip-ankle (IHA angle) and hipankle-toe (HAT angle), digitized using MaxTraq software with final excursions determined using a custom designed excel macro. Gait was assessed using the regularity index which is calculated as the number of correctly patterned plantar steps over the total number of steps [188]. BBB open field locomotor assessments [187] were done once a week on Fridays.

\section{Euthanasia and tissue histology.}

Animals were sacrificed using a ketamine $(50 \mathrm{mg} / \mathrm{kg}) / \mathrm{xylazine}(0.024$ $\mathrm{mg} / \mathrm{kg}) /$ acepromazine $(0.005 \mathrm{mg} / \mathrm{kg})$ cocktail and transcardially perfused [192] with phosphate buffer. The spinal cord was dissected out and postfixed in 4\% PFA overnight and cryoproteced in 30\% sucrose for at least 4 days. The injury level (T10) was confirmed visually using a dissection microscope, and the injury epicenter was blocked in tissue 
freezing medium. Transverse sections were cut at $30 \mu \mathrm{m}$ on a cryostat for histological assessment of spared white matter (SWM) at the SCI epicenter. The sections were stained with eriochrome cyanine and imaged at $4 \mathrm{X}$ using a light microscope. Dark blue compact white matter was traced and measured in ImageJ and the section with the lowest percentage of SWM was determined to be the epicenter [191].

Data is shown as mean \pm standard deviation. The outcome measures were analyzed for changes over time using RM ANOVA, followed by a Bonferroni post hoc t-test. Onesample t-test was used to compare regularity index values between baseline (100\%) and all time points post-injury.

\section{Results}

EMG responses to stretch.

Figure 9 shows representative EMG responses to stretch in the contralateral limb: clonic-like contraction (between 3 to $9 \mathrm{~Hz}$ ), air-stepping and spasms. Clonic-like responses and spasms were also present in the ipsilateral limb when it was stretched at week 8 , but air stepping could not be observed because the limb was held in a stretched position. These three responses were observed in all animals and are consistent with our previous observations $[171,254]$. The responses can be observed while the limbs are being positioned ("pre-stretch") and stretched and then typically decline while the limb is maintained in the stretch position for 1 minutes. The frequency and amplitude of these responses to stretch increased over time after SCI. Figure 10 shows EMG responses recorded while the limb was being positioned at week 2 (A) and week 8 in the contralateral limb (B) and ipsilateral limb (C). Figure 11 shows the EMG responses and recorded torque during a 1 minute quadriceps stretch of the contralateral limb at week $2(\mathrm{~A} \& \mathrm{~B})$ and in the ipsilateral limb at week $8(C \& D)$. EMG response amplitude and the frequency of clonic- 
like contractions were quantified for weeks 2 to week 8 (Figure 12). Although there were no significant differences over time there was an overall trend of increased amplitude and frequency in clonic-like responses from week 2 to week 8 during most stretches in both contralateral and ipsilateral limbs. Clonic-like contractions were particularly robust during quadriceps and tibialis anterior stretches.

Figure 9

A. Clonic-like response
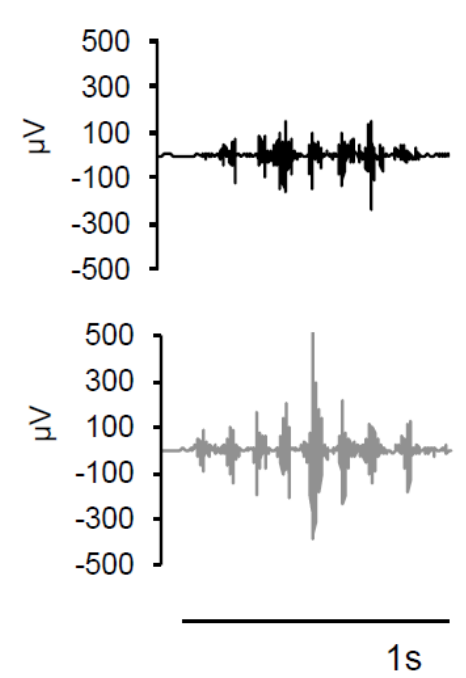

B.
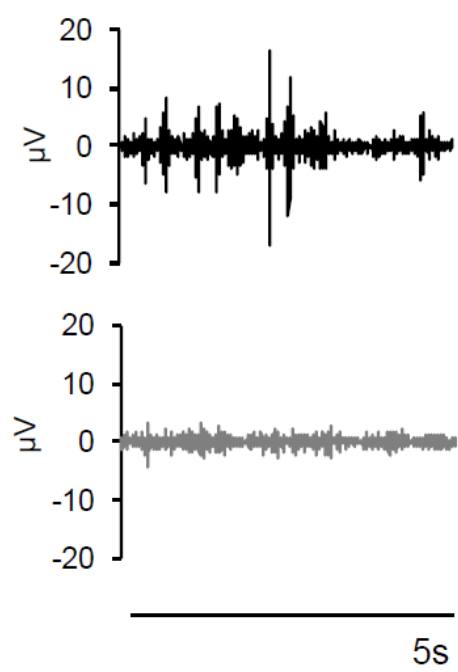

C. Spasm
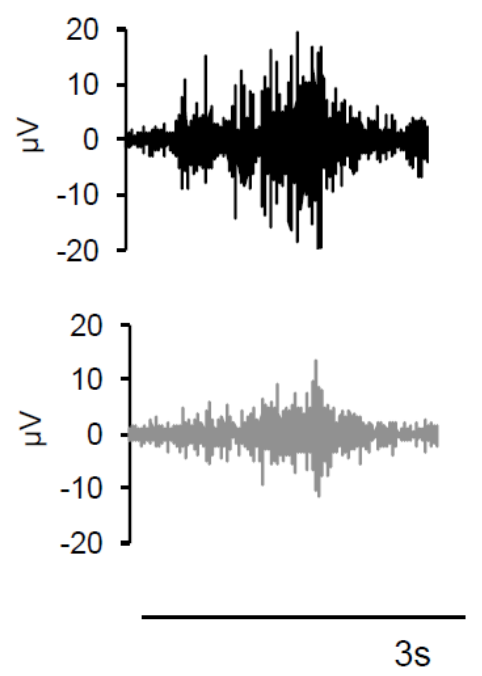

Rectus Femoris Biceps Femoris

Figure 9. Three commonly occurring EMG responses during hindlimb stretching knee muscles of the rats with SCI.

Top trace shows the response of Rectus Femoris and the bottom trace - Biceps Femoris of the contralateral limb: clonic-like contractions at frequencies 3-10 Hz (A), air stepping with predominantly extensor activity (B) and spasms (C) 
Figure 10

Rectus Femoris
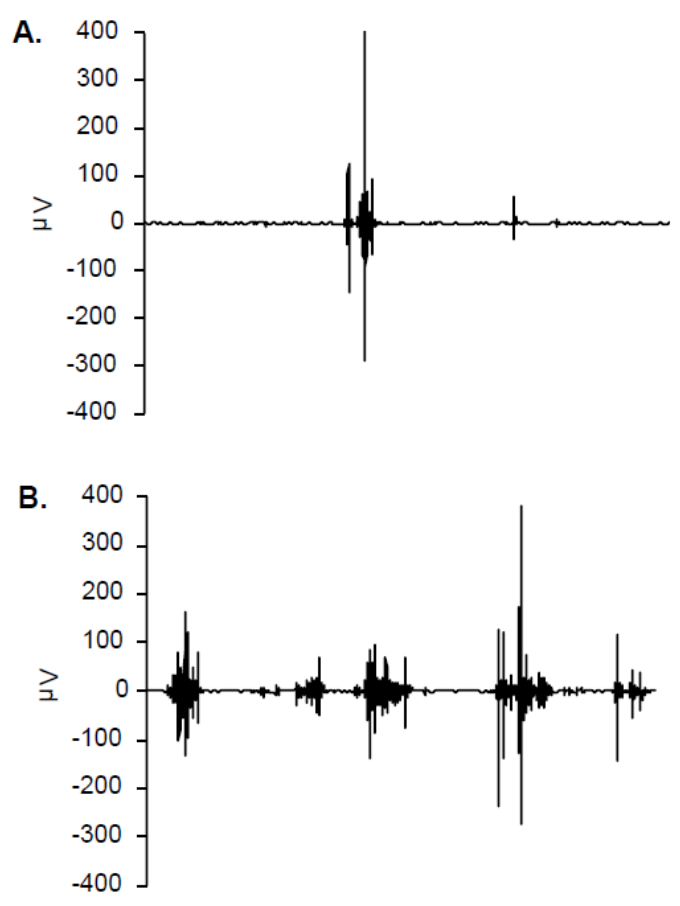

C.

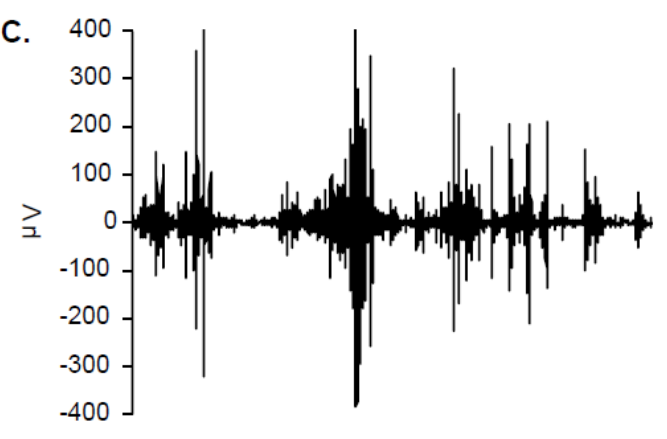

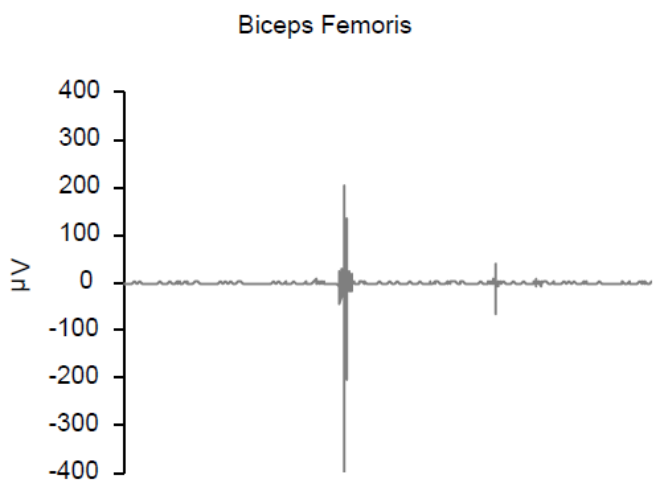
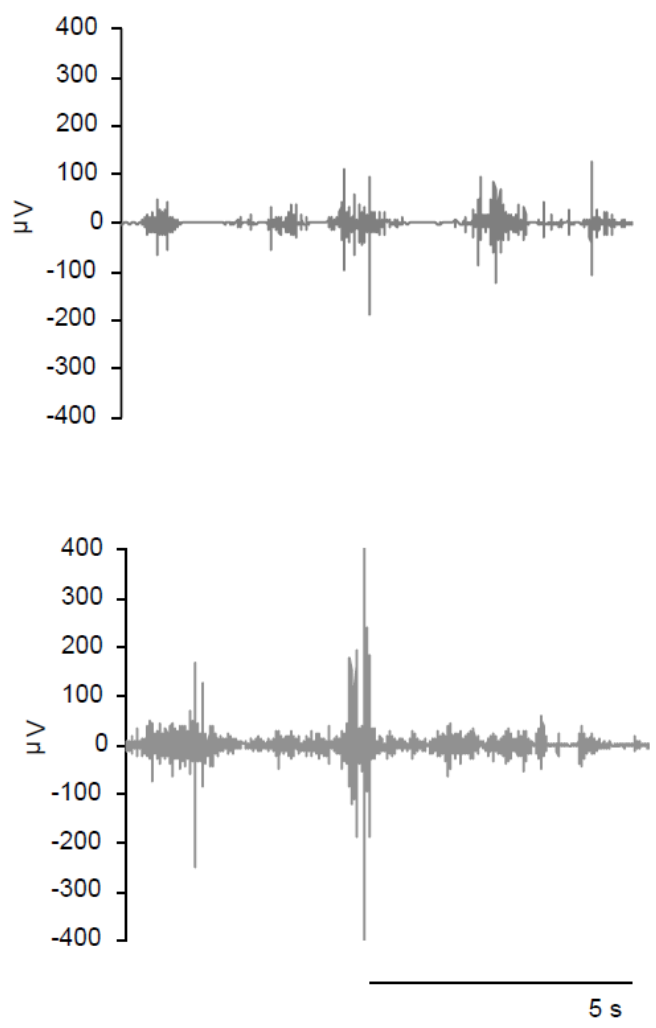

Figure 10. Examples of EMG responses during a 10 second positioning period prior to quadriceps stretch at 2 and 8 weeks post SCI.

A. EMG response in the contralateral limb at 2 weeks post-SCI. B. EMG response in the contralateral limb at 8 weeks post-SCI. C. EMG response in the ipsilateral limb at 8 weeks post-SCI. 
Figure 11

A.

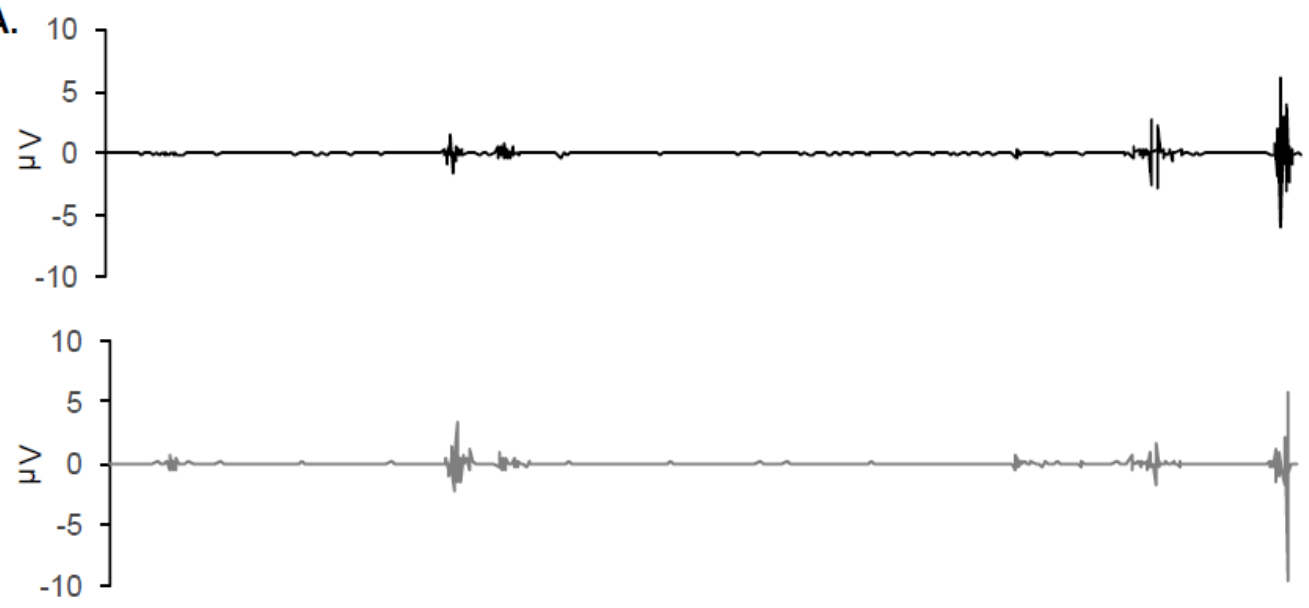

B. 3
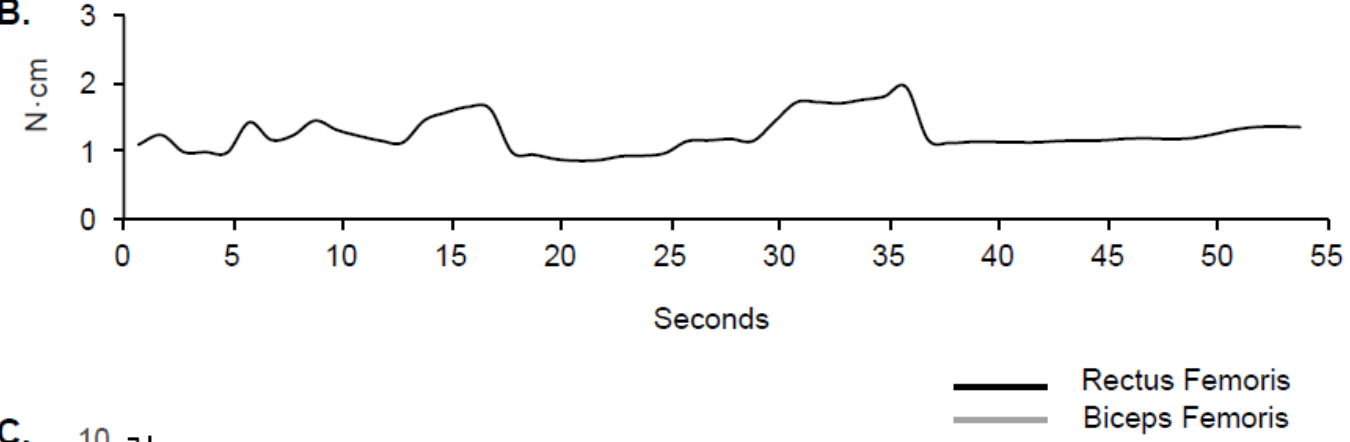

c.
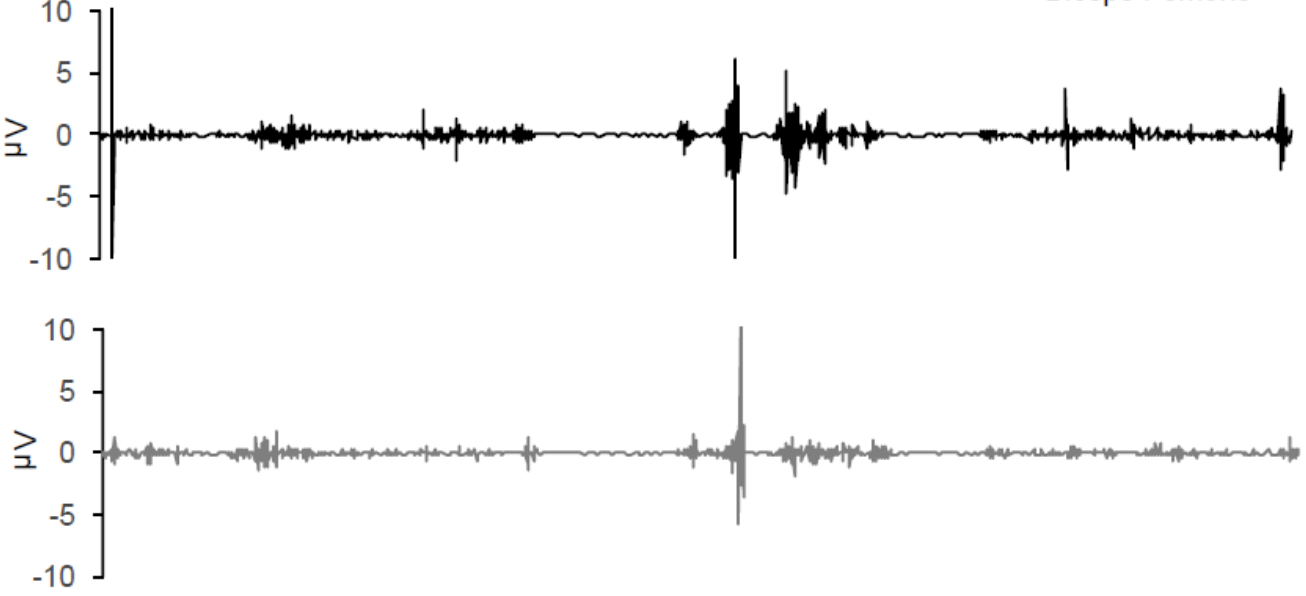

D.

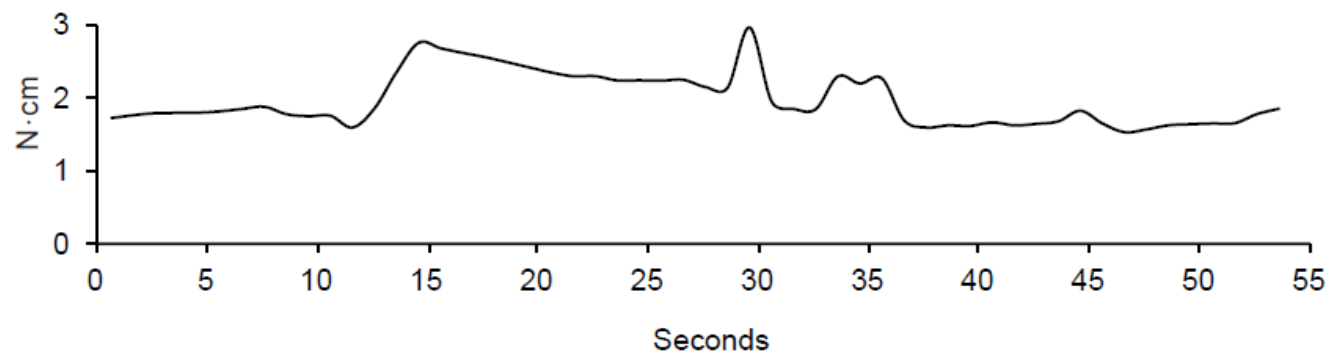


Figure 11. EMG and force traces during a 1 minute quadriceps stretch

A. Responses in the contralateral limb at week 2 post-SCI. C. Responses in the ipsilateral limb at week 8 post-SCI. B and D show forces applied over the duration of the stretch. In D forces increase when there is a spike in EMG activity, demonstrating that the force sensors were sensitive to the resistance from the animal when hindlimbs were activated. 
A.

Rectus Femoris

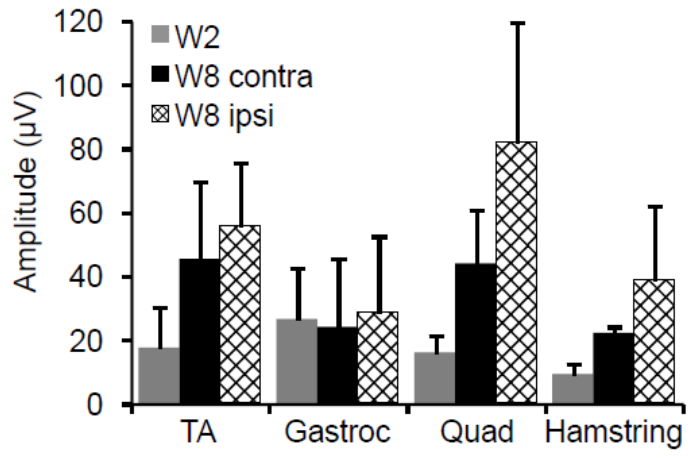

C.

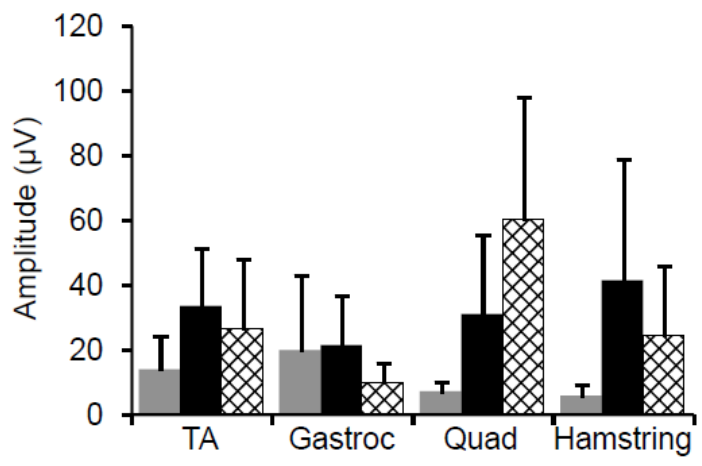

B.

Rectus Femoris

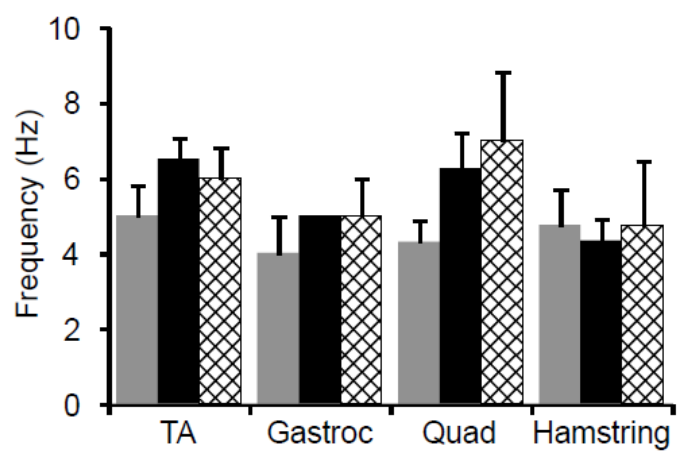

D.

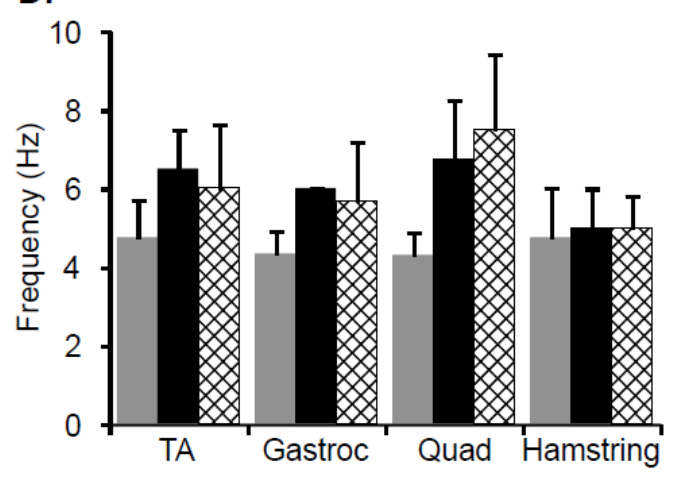

Figure 12. Quantification of amplitude and frequency of clonic-like EMG responses to stretch in the contralateral limb at week 2 and 8 , and ipsilateral limbs at week 8 .

Although there were no statistically significant differences due to small sample size $(n=4)$ and high variability, there was a trend in increased amplitude $(\mathrm{A}, \mathrm{C})$ and frequency $(\mathrm{B}, \mathrm{D})$ of clonic-like contractions from week 2 to 8 , most pronounced in responses to quadriceps and tibialis anterior stretch. 
Torques applied during stretching.

The torques (Fig.13) applied to achieve and end range-of-motion varied dramatically for the four muscles stretched, with the gastrocnemius requiring about 0.8 $\mathrm{N}^{*} \mathrm{~cm}$ and the tibialis anterior requiring about $6 \mathrm{~N}^{*} \mathrm{~cm}$. Overall the required forces were consistent over time for the Gastrocnemius (B), Hamstring (D) and TA stretches (A) with the exception of week 7 for the TA. However the forces required for quadriceps stretches gradually increased over time and were significantly greater at weeks 5, 6 and 8 as compared to week 2 (C) 


\section{Figure 13}

A. Tibialis Anterior Stretch

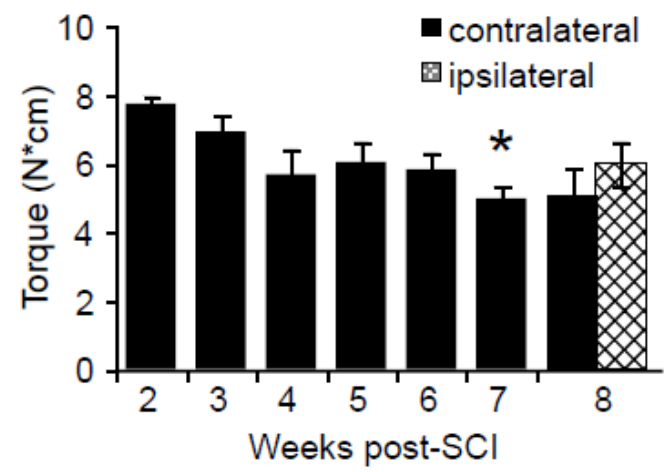

C.

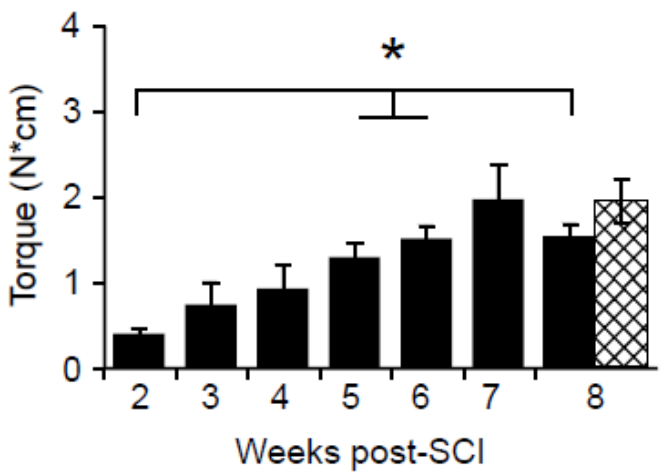

B. Gastrocnemius Stretch

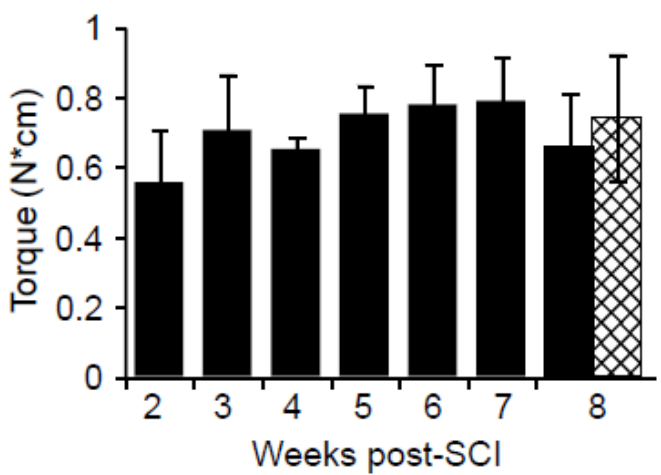

D. Hamstring Stretch

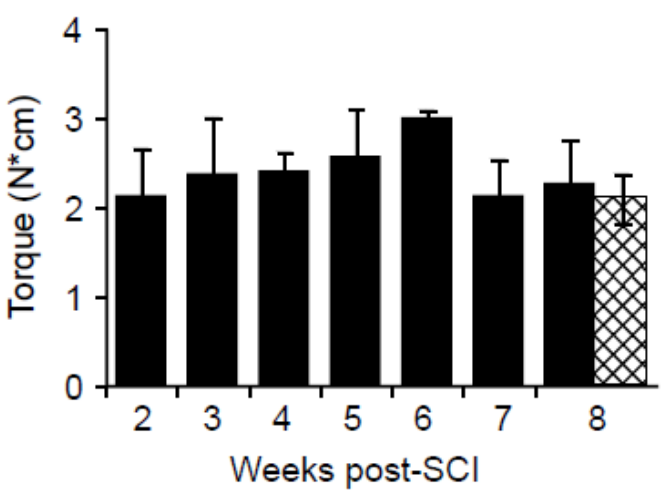

Figure 13. Quantification of weekly forces during the four hindlimb stretches.

The forces remained consistent through the weeks of stretching for Gastrocnemius (B), Hamstring (D) and Tibialis Anterior (A), except at week 7, when forces were significantly lower as compared to week $2, \mathrm{p}<.05$. The forces gradually increased with weeks post-SCI for the Quadriceps stretch (C) and were significantly greater at weeks 5, 6 and 8 as compared to week 2 . 


\section{Locomotor function.}

Figure 14 shows the weekly BBB score (A), regularity index (B) and joint excursions for the hip-ankle-toe (C) and iliac crest-hip-ankle (D). The EMG implant appeared to have no adverse effects on the locomotor function of the animals as they moved in the open field as all the animals had BBB scores of 21 post-implant. Spinal cord injury resulted in significant disruptions to locomotor function, however by week 2 post-SCI the animals were able to generate consistent weight supported stepping. The gait was significantly impaired for the first 5 weeks post-SCI as compared to baseline (Fig. 14B). The excursion of the HAT angle, representing the ankle and knee, significantly increased compared to baseline (Fig. 14C), while excursions of the IHA angle, representing the hip and knee, significantly decreased (Fig 14D). Stretching one limb twice a week had no obvious effect on the locomotor function of the animals over the initial 6 weeks. During weeks 7 and 8 when we began stretching the non-implanted limb and added ankle stretches of the implanted limb, BBB scores still remained stable, however, the BBB subscores dropped from an average of 4 to 2.2 during weeks 7 and 8 (data not shown). Pre and poststretch HAT and IHA angular excursions of the stretched limb were not significantly different. In addition, there was a trend towards gait impairment; the regularity index dropped from pre- to post stretch for each stretching session. These effects were only statistically significant at week 6, when pre-stretch RI values were identical for all animals. This low variability most likely allowed statistically significant differences to be detected despite the small sample size. 
Figure 14

A. Open Field Locomotor Scale

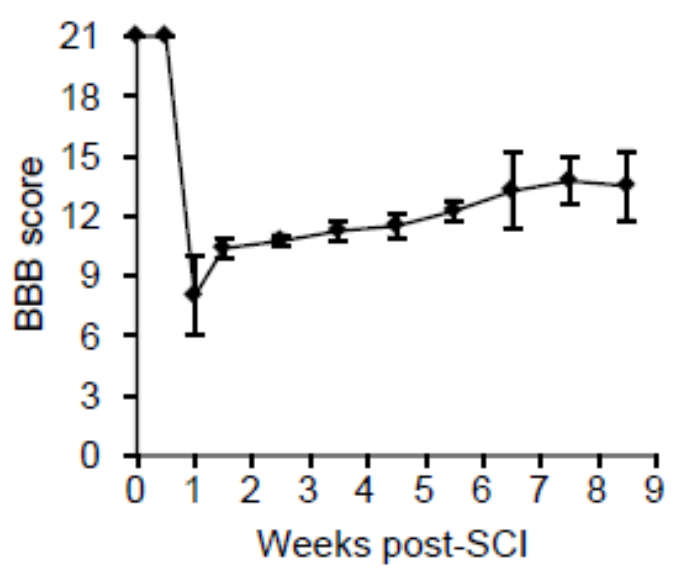

C.

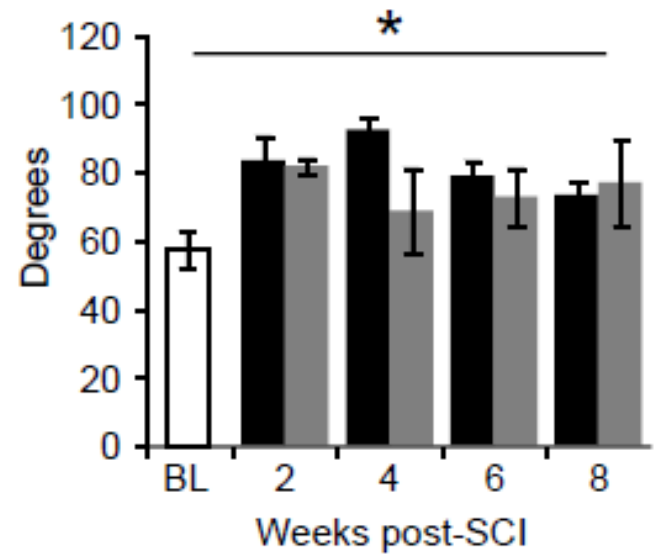

B. Regularity Index

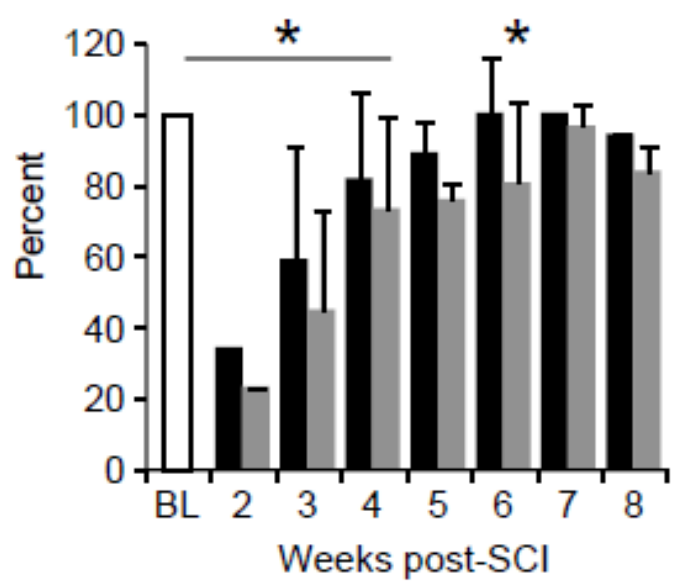

D.

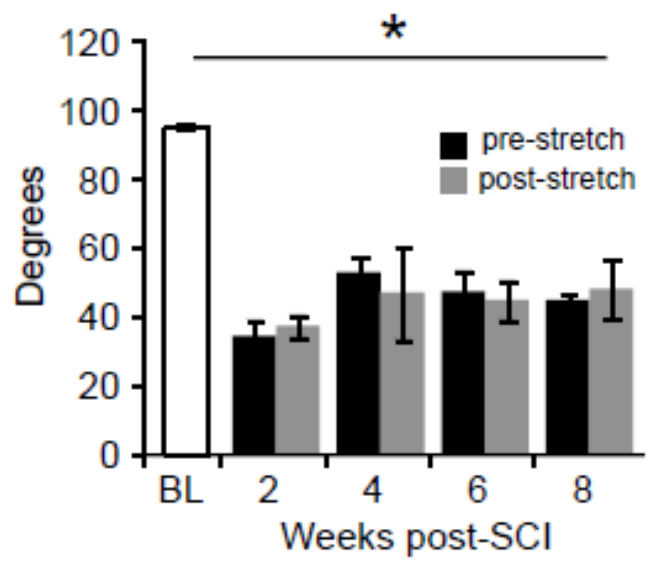


Figure 14. BBB Open Field Locomotor Scores, 3D kinematics and gait analysis of the animals after SCI and during stretching.

Spinal cord injury resulted in significant disruption of locomotor function. By 5 weeks the animals achieved significant recovery as the regularity index was no longer different from baseline (except week 6 post-stretch). There were no statistically significant difference in pre- and post- stretch gait regularity index (B) although there was a trend in slight decreases in RI post-stretch. The BBB scores remained stable for the duration of the study and stretching of one limb twice a week for 6 weeks and then daily stretching (all 6 stretches of the non-implanted limb and 2 ankle stretches of the implanted limb) during week 7 and 8 did not overall disrupt locomotor function (A). After SCI Hip-Ankle-Toe (HAT) (C) excursions were significantly increased while Iliac Crest-Hip-Ankle (IHA) (D) excursions were significantly decreased for the duration of the study. Pre- and post-stretch HAT and IHA excursions were not different (assessed using 3D kinematics). 


\section{Discussion}

Previously $[171,218,254]$ we observed that stretching hindlimb muscles after an incomplete thoracic spinal cord injury induces motor responses in both the stretched (ipsilateral) and unstretched (contralateral) hindlimb. These responses have been noted and referred to as air stepping, spasms and kicking. In the current experiment we determined that the "kicking" responses have a robust EMG pattern occurring at 3- $9 \mathrm{~Hz}$ frequencies that is similar in appearance and frequency to clonus in humans [255]. We observed these clonic-like contractions in response to stretch in both contralateral and ipsilateral limbs. Although clonus in humans with neurological impairments is most frequently seen in the stretched limb one study observed clonus also in the contralateral leg in SCI patients [256]. In our current study the clonic-like responses could be evoked at 2 weeks post-SCI, when we began stretching, however the number of responses and their amplitude and frequency increased by week 8 post. These increases were not significant due to the small sample size and variability. Clonic-like responses were most often observed simultaneously in both the knee flexor (Biceps Femoris) and knee extensor (Rectus Femoris), appearing as cocontractions, although less frequently we also observed clonic-like responses in one but not the other muscle. Co-contractions have also been reported during clonus in human subjects with SCI. Clonus in SCI patients can occur in response to stretch or other cutaneous inputs and significantly interferes with the activities of daily living [257]. We did not observe any spontaneously occurring clonic-like contractions while animals were in their cages or during kinematic and gait analysis, although spasms are sometimes observed during open field locomotor assessments. In general, it has been difficult to create a clinically relevant model of spasticity because animals with incomplete injuries develop only mild forms of hypereflexia that is difficult to detect without using velocity-dependent 
measures $[258,259]$. However, animals with complete transections at the sacral spinal cord level develop spasticity and tail clonus [260]. More recently, in a study by van Gorp et al., animals exhibited bilateral kicking in response to nociceptive mechanical stimuli applied to the unloaded hindpaw [261]. The authors suggest that these responses could be clonus. In the present study we demonstrate that stretching activates nociceptive afferents, thus it is possible that the clonic-like responses are the result of nociceptive signaling. Although clonus in humans is thought to be mediated through activation of Ia afferents [262], a study of human subjects with SCI showed that myoclonus could no longer be elicited when the underlying pathology (in spinal nerve roots, hip joint, thigh muscle) was resolved [55]. Presumably, nociceptive afferent activation subsequent to peripheral tissue damage in these patients triggered clonus or indirectly contributed to clonus evocation by increasing the excitability of the circuitry.

Air stepping is another consistent pattern that we see during stretching. Frequently, robust clonic-like movements at the initiation of stretch were replaced with air stepping that varied in intensity and the number of hindlimb segments involved. With more intense air stepping we saw the EMG bursts with greater activation in Rectus Femoris, but in most cases, air stepping although rhythmic and frequent did not result in detectable EMG in the knee muscles. Consistently with previous observation, spasms frequently occurred at the end of a 1 minute stretch. Sometimes clonic-like contractions had spastic like appearance, with wider less consistent peaks within the clonic episode. Early on after injury we also observed spasms that were quite subtle in appearance, almost like an isometric contraction - increased EMG in the knee muscles of the contralateral limb but only slight movements in the toes were apparent while a static stretch was maintained on the opposite limb. 
In this experiment we used a custom designed force glove to measure forces during the four major stretches. We found that the highest forces applied during tibialis anterior stretch were on average around $6 \mathrm{~N}^{*} \mathrm{~cm}$, while the lowest forces of $0.8 \mathrm{~N}^{*} \mathrm{~cm}$ were applied during the Gastrocnemius stretch. One of the limitations of the force sensors used in the current experiment is that the accuracy of their force measurement was somewhat dependent on the precise localization of the center of the sensor to the point of the highest force application. Tibialis Anterior stretch was perhaps the easiest to achieve such precise positioning of the force sensors, as the index finger sensor was placed on the heel of the rat (a very distinct landmark which was smaller than the sensor itself) and the thumb was placed on the dorsal part of the foot. In addition, end ROM of the ankle plantarflexion is achieved more readily as compared to the other stretches and therefore there is higher resistance from the tissues at the end ROM of the ankle plantarflexion which could also explain the highest forces were achieved during this stretch. While the forces during TA, Gastrocnemius and Hamstring stretches were consistent throughout the weeks, there was a gradual increase in forces during the Quadriceps stretch. As animals recovered from SCI, greater number of clonic-like contractions, particularly during the quadriceps stretch, likely contributed to the increase in torque over time, since the force sensors are sensitive to the resistance coming from the animal during the stretch.

To our knowledge, the current experiment is first animal study to conduct force measurements during stretching in rats (besides the previous experiment in the lab for a master thesis project - the development of the force glove, unpublished data). A few studies have addressed the issue of forces applied during stretching in human patients with SCI. Harvey et al., quantified the torques applied during hamstring stretch by twelve different 
PTs [211]. The range of forces varied substantially with the median torques in the range of 30-68 $\mathrm{N}^{*} \mathrm{~m}$, while some therapists applied torque over $100 \mathrm{~N} * \mathrm{~m}$. If we compare torques about the hip for human and rat based strictly on body weight (average weight of subjects from Harvey's study $-73 \mathrm{~kg}$, and average weight of the rats in our study $-.25 \mathrm{~kg}$ ) the ratio would approximately be $290: 1$. The equivalent range of torques for rats during the hamstring stretch would be $10-23 \mathrm{~N}^{*} \mathrm{~cm}$. In this study, the average torque during hamstring stretch was around $3 \mathrm{~N}^{*} \mathrm{~cm}$. In another study by Harvey et al., examining a 4 week stretching intervention patents' ankles were stretched (gastrocnemius stretch) using a set torque of $7.5 \mathrm{~N} * \mathrm{~m}$ while ROM assessment was performed using $10 \mathrm{~N} * \mathrm{~m}$ torque [94]. Once again, equivalent torques for rats would be 2.8 and $3.8 \mathrm{~N} * \mathrm{~cm}$. The average torques applied during gastrocnemius stretch in the current experiment were $0.8 \mathrm{~N}^{*} \mathrm{~cm}$. This comparison has obvious limitations, for example, it is not known if rat's sensitivity to forces is comparable to humans, however, the torques applied in this study are still well under those reported for human patients for these stretches.

The primary goal of the current study was to determine the EMG responses of the animals to stretch. However, some observations regarding the effects of stretch on locomotor function were made. Stretching of one limb for the first 6 weeks did not result in overall disruption to the locomotor function as in previous studies [165, 171, 218, 254]. There was a drop in BBB subscores (reduction in toe clearance and increased paw rotation) at the end of the weeks when stretching was done every day (week 7 and 8), however. In addition there were slight drops in the regularity index after every stretching session which occurred most likely due to increased number of dorsal steps. This pattern of subtle disruption taken together with drops in BBB subscores suggests that the "low" dose of 
stretching used in this experiment had an effect on finer motor control aspects of locomotion. This data provides small but informative details to help us further understand the stretching phenomenon. Perhaps, these effects of intermittent stretching in high functioning animals with mild-moderate injuries $(20 \% \mathrm{SWM})$ are comparable to the negative effects of stretching on some aspects of motor performance (isometric strength, muscle strength endurance, isokinetic torque production, etc) seen in intact human subjects $[220,225,227]$.

In conclusion, we have identified at least two similar EMG responses elicited by hindlimb stretching in rats as reported for human subjects: clonic-like contractions and spasms. We also determined that the torques used during stretching of rat hindlimbs are comparable and likely even lower than those PTs apply to human patients. Although it would be ideal to design a similar experiment in patients with SCI in order to directly compare the findings between human and rat, the existing evidence strongly suggests that stretching might have similar effect on the nervous system in humans as it does in rats. Therefore, further investigation of stretching and its effects on motor function in human subjects strongly warranted. 


\section{CHAPTER VI \\ ROLE OF NOCICEPTIVE AFFERENTS IN STRETCH-INDUCED LOCOMOTOR DEFICITS IN RATS WITH SPINAL CORD INJURIES}

\section{Introduction}

The most obvious manifestation of a severe spinal cord injury (SCI) is an immediate paralysis of musculature below the level of lesion and subsequent loss of motor function. Patients with severe SCIs become dependent on wheelchairs for their mobility. Lack of active loading and/or weight bearing in the limbs results in muscle atrophy and pathological changes in joints and their supporting structures that with time can result in muscle contractures - dramatically decreased range of motion (ROM) and increased stiffness [66, 263]. In addition, neurobiological changes within the spinal cord below the level of injury leads to the development of spasticity [54]. Stretching remains one of the leading therapies for the prevention and treatment of muscle contractures as well as spasticity $[179,181]$. Rationale for therapeutic stretching largely comes from animal studies of joint contractures that develop as a result of cast immobilization of muscles in shortened positions of otherwise neurologically intact animals. Intermittent stretching in these studies at was effective at preventing ROM decreases during cast-based limb immobilization [68, 87, 88]. In addition, stretching is the cornerstone of any flexibility training program and is effective at increasing ROM acutely after a single 30-90 second stretch $[78,79]$ and chronically, after multiple weeks of flexibility training utilizing various frequencies and durations of 
stretching in healthy intact human subjects [81-83].Unfortunately, several systematic reviews found no evidence to support efficacy of stretching at preventing muscle contractures or improving ROM after SCI and other neurological conditions [97, 98].

With the help of trained and licensed physical therapist (PTs) we developed a hindlimb stretching protocol in an attempt to treat muscle contractures in wheelchairimmobilized rats with mild SCIs [165]. Surprisingly, non-immobilized rats with SCIs that received stretching as a contracture-control measure had significantly reduced locomotor recovery as compared to unstretched, non-immobilized controls. We now have demonstrated that stretching at chronic time points results in dramatic reductions in locomotor function in rats with more severe injuries [218]. Furthermore, we showed that dynamic (ie. rhythmic) hindlimb stretching also disrupts locomotor function. However, the physiological mechanisms underlying the stretching phenomenon are not known. We have been unable to find signs of overt muscle damage [218] that could account for the locomotor dysfunction. Animals show a robust and rapid (1-2 weeks) recovery when brief periods (1-4 weeks) of daily stretching cease. Thus, it appears feasible that the stretchinduced locomotor deficits likely have a sensory-dependent neurologic basis. Interestingly, skeletal muscles receive a robust sensory innervation. For example, the sciatic nerve, which is a major source of hindlimb innervation, is predominantly composed of sensory axons, $48 \%$ of which are unmyelinated sensory afferents (C-fibers and group IV fibers)[264]. A study of 15 cat skeletal muscles determined that group IV sensory afferents comprise about $66 \%$ of the total sensory innervation [265]. Group III and IV afferents have been shown to mediate decreased motor output during fatiguing exercise in humans [266], and Cleland et al., showed that activation of stretch sensitive free nerve endings (group III and IV 
afferents) produces rapid inhibition of motor output [203]. Therefore, we hypothesized that the negative effects of stretching are mediated wholly or partly by nociceptive afferents. We propose that stretching will not be detrimental to the locomotor function of spinal cord injured rats that are systematically depleted of unmyelinated afferents using neonatal capsaicin treatment.

\section{Methods}

Ethical statement concerning animals research.

All experimental procedures involving animals were approved by the University of Louisville Institutional Animal Care and Use Committee.

\section{Neonatal capsaicin injections.}

In order to test our hypothesis we turned to the well-established method for depleting nociceptive afferents, neonatal capsaicin injections [267, 268]. Six pregnant Sprague-Dawley rats of known gestational age were checked several times a day to ensure an accurate record of birth time. Every pup in two of the litters received capsaicin injections, pups in two other litters received vehicle injections and the remaining two received no injections. Injections were done at 2 days of age. The rat pups were taken out of their cages (half of the litter at a time, along with some bedding) and received intraperitoneal injections of either capsaicin $(50 \mathrm{mg} / \mathrm{kg}$ ) dissolved in 10\% Tween 80 and $10 \%$ ethanol $(\mathrm{v} / \mathrm{v})$ in $0.9 \%$ saline or vehicle injections (same solution without capsaicin). Anesthesia was achieved by wrapping pups in gauze and placing them on ice for approximately 5 minutes. The animals were monitored continuously and the injections were done when all movements ceased and animals were not responsive to touch or pinch. After the injection, the animals were placed on the removed bedding until they warmed up and regained movement. The animals were returned to their mothers for the next 4 weeks 
after which they were weaned. The weaned animals were sexed so that males and female could be housed separately. Only female rats were used in the current study. The animals were allocated to 3 groups: capsaicin-treated (CAP, $n=8)$, vehicle-treated $(V E H, n=8)$ and control animals that received no injections $(\mathrm{CON}, \mathrm{n}=8)$. All three groups received SCI; CAP and VEH rats received stretching, while animals in CON group served as controls and were not stretched. Gentling procedures and baseline assessments began when animals were 3 months old. All of the following assessments, stretching and data analysis were done by individuals blinded to the experimental groups.

\section{Baseline sensory assessments.}

To assess the effectiveness of TRPV1+C-fiber depletion by capsaicin, animals were tested for withdrawal thresholds to painful stimuli using electro-von Frey (mechanical) and Hargreaves (thermal) sensory tests, as described in detail previously [269]. Briefly, for von Frey testing, the animals were placed on a metal grid and an electrovon Frey rigid filament was applied to the plantar portion of the foot. The force recorded was that registered at the point of paw withdrawal when followed by a stereotypic behavioral response of attending to the stimulated foot. For the Hargreaves test, the animals were placed on a heated glass surface and a laser $\left(32^{\circ} \mathrm{C}\right)$ was shone onto the plantar surface of the foot. The latency of paw withdrawal from the stimulus was recorded.

C-fiber depletion was also assessed using the cutaneous trunci muscle reflex (CTMR), which is purely nociceptive in rats [270]. CTMR testing was performed following administration of a sedative dose of sodium pentobarbital $(35 \mathrm{mg} / \mathrm{kg})$. A five by three centimeter grid of black dots was drawn on the skin of the back starting from the midline ( $3 \mathrm{~cm}$ from the base of the tail). The midline dots were $1 \mathrm{~cm}$ apart (rostrocaudally) 
and $5 \mathrm{~mm}$ apart mediolaterally (a schematic drawing of the grid is shown in Fig. 1C). Mechanical (forceps pinch) and thermal (metal probe heated to $65^{\circ} \mathrm{C}$ ) were applied at two sites bilaterally. Digital video recordings of the skin were made with a camera placed directly above the animal. MaxTrack software was used to digitize the dots and to quantify contraction distance and time to maximal contraction using two dots rostral to the stimulation sites.

Baseline locomotor function assessment.

Hindlimb 3D kinematics were recorded using two side view cameras while animals walked in a narrow Plexiglass tank. Using MaxTrack program and custom-designed excel macro, we analyzed the excursions of two angles within the three major hindlimb segments (hip, knee and ankle): hip-ankle-toe and iliac crest-hip-ankle as previously described [188], in an effort to avoid inaccuracies associated with the knee measurement, we analyzed the limb as three segments and two angles: the iliac crest-hip-ankle and the hip-ankle-toe angles.

Magnetically evoked muscle potentials.

Electromyographic (EMG) responses were recorded from gastrocnemius muscles following magnetic stimulation of afferent fibers at the base of the tail as described previously $[171,218]$. Unanesthetized animals were gently but securely restrained using a cloth stockinet pinned to a wooden board. Twenty six gauge needle electrodes were inserted into the gastrocnemius muscles bilaterally. The stimulus $(80 \%$ of maximum intensity) was delivered to the base of the tail using a $25 \mathrm{~mm}$ "figure 8 " magnetic coil powered by a MagStim 200 (MagStim Ltd., Whitland, U.K.). The EMG responses were 
analyzed for onset latency and peak-to-peak amplitude. Assessments were performed preinjury and at week 4 and 11 post SCI.

\section{Spinal cord injury.}

Spinal cord injury was performed as previously described [185]. Animals were anesthetized with ketamine/xylazine and a midline incision was made over the lower thoracic spine, followed by partial laminectomy at $\mathrm{T} 9$ to expose the T10 spinal cord. Moderately-severe spinal cord contusions $(25 \mathrm{~g} / \mathrm{cm})$ were delivered using the NYU "MASCIS" Impactor (W. Young, Rutgers University, Piscataway, NJ).

\section{Locomotor assessment after SCI and stretching.}

Locomotor function was assessed using the BBB Open Field Locomotor Scale [187] weekly for the first 6 week. During the weeks of stretching, BBB scores were assessed 3 times a week, Monday am (pre-stretch), Monday pm (after one stretching session) and Friday pm (after 5 days of stretching), as previously described [171, 218]. In addition, partial weight-supported stepping was assessed using shallow water ( 2 inches) every second week. Horizontal and ventral views of eight to ten passes (uninterrupted movement the full length of the tank) were recorded for each animal. Hindlimb steps, defined as a movement involving clear foot contact (either dorsal or plantar) with the pool surface at initiation followed by an active propulsion phase (body movement forward relative to a planted and stationary foot). In other words, a full step cycle consisting of both swing and stance phases had to be present for a step to be included in the analysis. For each identified step we calculated the excursions of the HAT and IHA angles using MaxTraq software. The data is presented as the ratio of steps to passes for each animal, normalized to their pre-stretching (week 4) ability. 


\section{Stretching protocol.}

Our standard hindlimb stretching protocol [171] was initiated at 6 weeks post SCI when BBB scores had plateaued and locomotor function was stable for 3 weeks. The protocol consists of two 12 minute sessions of 6 stretches (each held at the end range of motion for 1 minute) applied to major hindlimb muscle groups bilaterally (ankle, knee, hip flexors/extensors and hip adductors and abductors). For the stretching sessions, animals were gently wrapped in a towel, leaving their hindlimbs exposed, and placed on their backs. Seven rat PTs, who were blinded to experimental groups participated in the daily stretching sessions and animals were rotated through the PTs so that they were not stretched by the same PT more than twice in a week. During each stretch the PTs closely monitored the animals and took notes of any responses that the animal displayed during each stretch in the "stretch response" form. Some of the most common responses are kicking (vigorous movement of the entire hindlimb), vibrations (low range of motion movement mostly around the ankle) [254], pull back (hindlimb withdrawal) and air-stepping (slow rhythmic step-like movement of the contralateral hindlimb). The responses were given a score of 1 , 2 or 3 based on intensity and frequency during each stretch: $1=$ mild/infrequent $(5-20 \%$ of the stretch time), $2=$ moderate, frequent $(20-70 \%), 3=$ severe/very frequent $(70-100 \%)$. Control animals were handled daily: they were wrapped in a towel and placed on their backs, but not stretched. Animals were stretched 5 days a week for 3 weeks and then were allowed to recover for 2 weeks. The rats were then stretched for an additional 2, 3 or 4 days and were sacrificed 2 hours after the last stretching session. Three animals from each stretch group (CAP and VEH) were sacrificed each day. Control animals were all sacrificed 2 hours after being wrapped in a towel and handled. All the animals were assessed using 
the BBB Open Field Locomotor Scale between the final stretching session and the time of sacrifice.

Sacrifice and tissue processing.

The animals were overdosed with ketamine $(50 \mathrm{mg} / \mathrm{kg}) /$ xylazine $(0.024$ $\mathrm{mg} / \mathrm{kg}) /$ acepromazine $(0.005 \mathrm{mg} / \mathrm{kg})$ cocktail and transcardially perfused with phosphate buffer saline (PBS) followed by 4\% PFA as previously described [192]. The spinal cord including the injury epicenter and lumbar enlargement was dissected out and post-fixed in 4\% PFA for one hour and transferred to $30 \%$ sucrose for cryoprotection for at least 4 days. The spinal cords were examined under the dissection microscope and it was confirmed that all of the lesions were at the T10 level by counting segments beginning in the lumbar section as levels can be easily determined based on the size of the dorsal root ganglions. A $12 \mathrm{~mm}$ length of each cord containing the injury epicenter was isolated, placed in a block with tissue freezing medium completely covering the sample and rapidly chilled to freezing on dry ice. Transverse $30 \mu \mathrm{m}$ sections were cut and stained with eriochrome cyanine (EC) and were photographed at $4 \mathrm{X}$ as described previously. Spared white matter (SWM) was assessed using the cross-sectional area of darkly stained, compact tissue, traced using ImageJ (NIH) and compared to unininjured animals (control) sections. The section with the least SWM was assigned as the injury epicenter. The lumbar spinal cord (L1-L5) was cryoprotected and sectioned at $20 \mu \mathrm{m}$ for immunohistochemical (IHC) analysis of c-Fos and calcitonin gene related peptide (CGRP). Three hindlimb muscles (Tibialis Anterior, Medial Gastrocnemius and Biceps Femoris) were also dissected out for histological analysis. Muscle length (origin to insertion) was measured, the muscle was cut approximately at mid belly and a $5 \mathrm{~mm}$ length (proximal to the muscle origin) was post- 
fixed in 4\% PFA and cryoprotected in 30\% sucrose. Each muscle sample was sectioned at $10 \mu \mathrm{m}$ and stained with hematoxylin and eosin (H\&E). The muscle was analyzed for the presence of centralized nuclei and fiber cross sectional area as previously described [218]. Photomicrographs of four muscle areas from each region (proximal, distal, medial and lateral within the cross section) were acquired at 20X. The number of muscle fibers (MF) containing centralized nuclei $(\mathrm{CN})$ were counted and expressed as a percentage of the total number of MFs analyzed. In addition, over 150 muscle fibers were traced from each muscle and CSA area determined using ImageJ software.

c-Fos immunohistochemistry and analysis.

Nuclei positive for c-Fos were immunolabeled using avidin-biotin peroxidase [271]. Slides with sections of lumbar spinal cord were warmed and washed with PBS. Endogenous peroxidase activity was quenched using 3\% hydrogen peroxide ( $15 \mathrm{~min})$. The sections were then blocked with $10 \%$ normal donkey serum (NDS) and $10 \%$ bovine serum albumin (BSA) in $0.3 \%$ PBS-Triton (PBST) for 1 hour. The sections were incubated with c-Fos primary antibody (mouse monoclonal (2H2), 1:1000, Abcam, ab208942) overnight at $4^{\circ} \mathrm{C}$. Sections were then rinsed with PBS/PBST, incubated with biotinylated antibody (donkey-antimouse, 1:1000, Jackson, 715-065-151) for 1 hour, rinsed with PBS/PBST, incubated with horseradish peroxidase-streptavidin (1:1000 in PBS, Vector Laboratories, SA-5004) for 1 hour and rinsed with PBS/PBST. The reaction sites were visualized by applying HRP-DAB solution (SigmaFast tablet kit, SLBJ7196V) for 10 minutes. The reaction was stopped by rinsing the slide with distilled water and the slides were air dried for at least 10 minutes, followed by 3 minutes in Xylene. The slides were coverslipped using paramount. Images of each section were acquired on an inverted microscope. Using 
ImageJ the gray matter was divided into three regions of interest (bilaterally): Subtantia Gelatinosa (SG), dorsal horn (DH) and intermediate gray matter/ventral horn (IGM\&VH). Darkly-stained, c-Fos positive nuclei were counted manually in ImageJ by an individual blinded to experimental groups. Three images for each lumbar level (L1-L5, approximately $800 \mu \mathrm{m}$ apart) were acquired and an average number of neurons from the three sections for each animal was taken into group average analysis.

\section{CGRP immunohistochemistry and analysis.}

IHC was performed to identify CGRP on sections of L3 spinal cord. Slides were warmed, rinsed and incubated in serum buffer (10\% NDS, $10 \%$ BSA in $0.3 \%$ PBST) for 1 hour to block non-specific antibody binding. Sections were incubated with anti-CGRP primary antibody (rabbit, 1:2000, Millipore, AB15360) overnight at $4{ }^{\circ} \mathrm{C}$, rinsed and incubated with secondary antibody (donkey-antirabbit, Alexa 467, Jackson, 711-606-152). The sections were coverslipped with fluoromount. Both left and right dorsal horns from three different sections for each animal were imaged at 20X. The area of CGRP-positive puncta within the dorsal horn was identified and differentiated using the threshold tool within Elements software, and the same threshold range was then applied to the rest of the images for all animals. The area of CGRP was quantified for each dorsal horn area excluding (bilaterally) the dorsal root entry zone. All these analyses were performed by an individual blinded to the experimental groups.

\section{Statistical Analysis.}

Data with multiple comparisons was analyzed using ANOVA followed by Bonferroni or Tukey HSD post hoc test. Data analyzed using ANOVA is reported as means \pm standard deviation (SD). For non-parametric analysis Mann-Whitney U and Spearman's 
rho tests were used. Significance was established when $\mathrm{p}<.05$; Figure legends indicate which test was used for the represented set of data.

\section{Results}

Baseline sensory and locomotor function.

Neonatal capsaicin treatment had no significant effect on responses to noxious mechanical stimuli. Paw withdrawal thresholds to von Frey filament stimulation were similar for the CAP, VEH and CON groups $(\mathrm{F}=.065, \mathrm{df}=2,21, \mathrm{p}=.937)$ (Fig. 15A). There were also no significant differences in contraction distance of the CTMR in response to forceps pinch at either site of stimulation, $A(F=1.862, \mathrm{df}=2,20, \mathrm{p}=.181)$ or $\mathrm{B}(\mathrm{F}=.798$, $\mathrm{df}=2,20, \mathrm{p}=.464$; Fig 15D). Unexpectedly, the CAP group had an increased speed to maximal contraction in response to the mechanical stimulus applied to site $B(p<.05)$ (Fig. 15E). CAP animals had increased latency to paw withdrawal in response to the heat stimulus of the Hargreave's test as compared to VEH animals $(\mathrm{p}<.05)$ (Fig 15B). As expected, in the CAP group the CTMR in response to the thermal probe $\left(65^{\circ} \mathrm{C}\right)$ was practically abolished: Contraction distance (Fig. 15F) was significantly decreased $(\mathrm{p}<.001)$ accompanied by significant reduction in speed of contraction (Fig. 15G) as compared to $\operatorname{VEH}(\mathrm{p}<.05)$ and CON $(\mathrm{p}=.005)$.

All animals had normal locomotor function while moving in the open field (BBB scores of 21, subscores of 13). In addition, the hip-ankle-toe and iliac crest-hip-ankle angles showed the typical excursion patterns of normal stepping (80 to 160 degrees of motion) and there were no group differences (Fig.16) 
A. von Frey Test B. Hargreaves Test C. CTMR Schematic
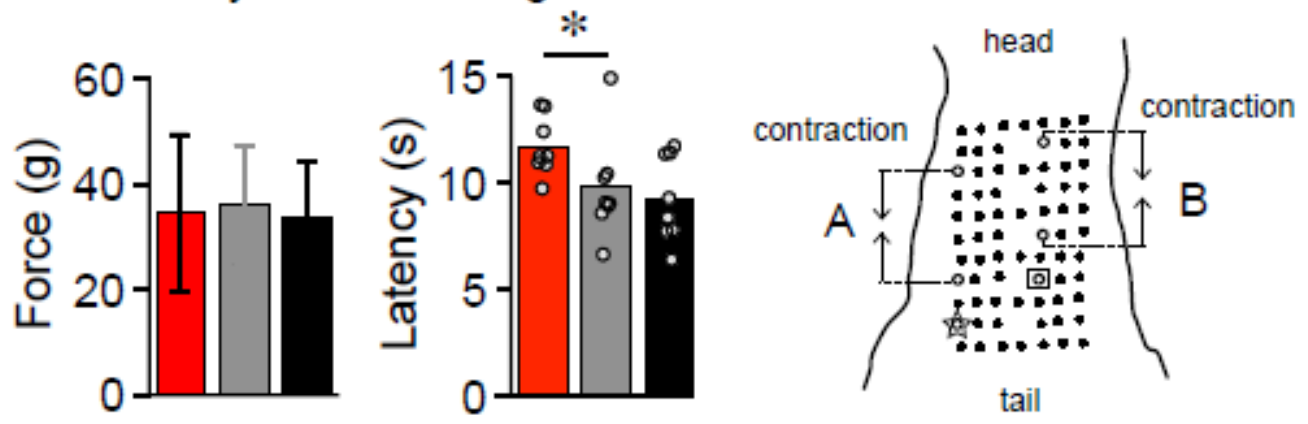

tail

D. Contraction Distance E. Speed to Minimal Contraction
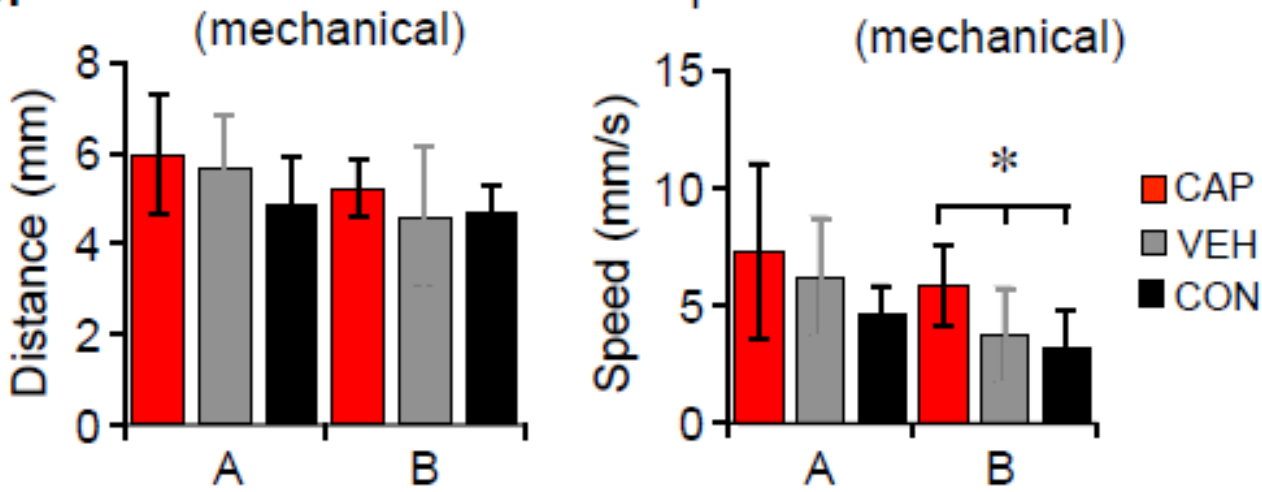

F. Contraction Distance (thermal)

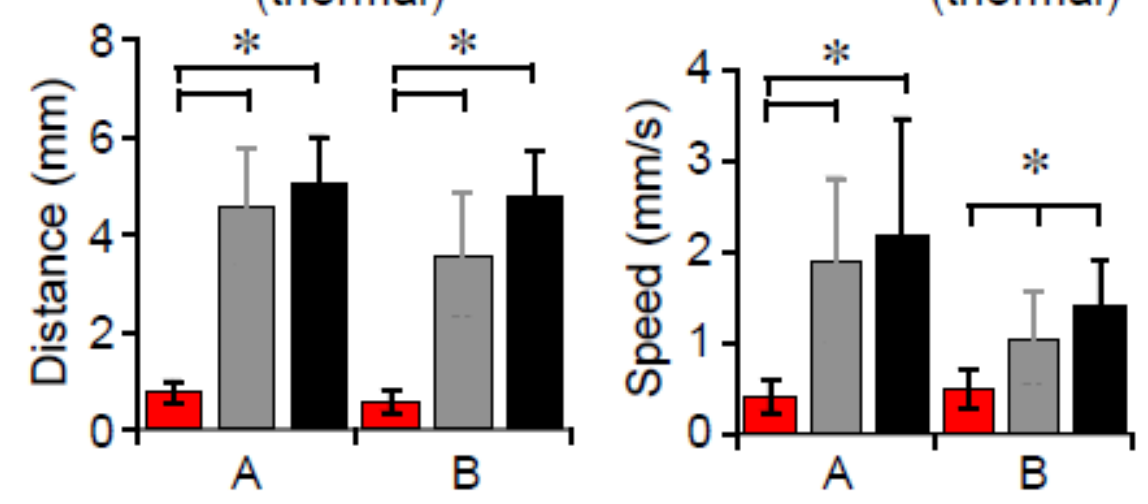

G. Speed to Minimal Contraction (thermal) 


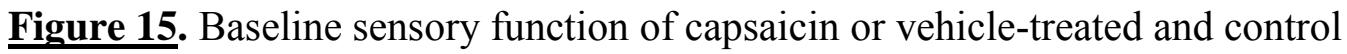
(untreated) animals.

Assessments were done using von Frey (A), Hargreaves (B) and Cutaneous Trunci Muscle Reflex (CTMR, D-G). C. shows a schematic of CTMR markings on the dorsal skin for quantitative analysis of the reflex in response to mechanical (forceps pinch) or thermal (heated metal probe) stimulus. Dot A (star) and B (square) are two sites for stimulus application on either side of the midline. The data for contraction distance (mechanical D, thermal $-F$ ) and speed of contraction (mechanical - E, thermal - G) between the two hollow dots rostral to the stimulation sites are shown. Data reported as means $\pm \mathrm{SD}$ for $\mathrm{A}$, D-G (One-way ANOVA, Tukey HSD post hoc, $\mathrm{p}<.05$ ), for B. bars represent means, dots each individual animal ( $\mathrm{n}=8$ in each group) (Mann Whitney $U$ test, $\mathrm{p}<.05$ ) 
Figure 16

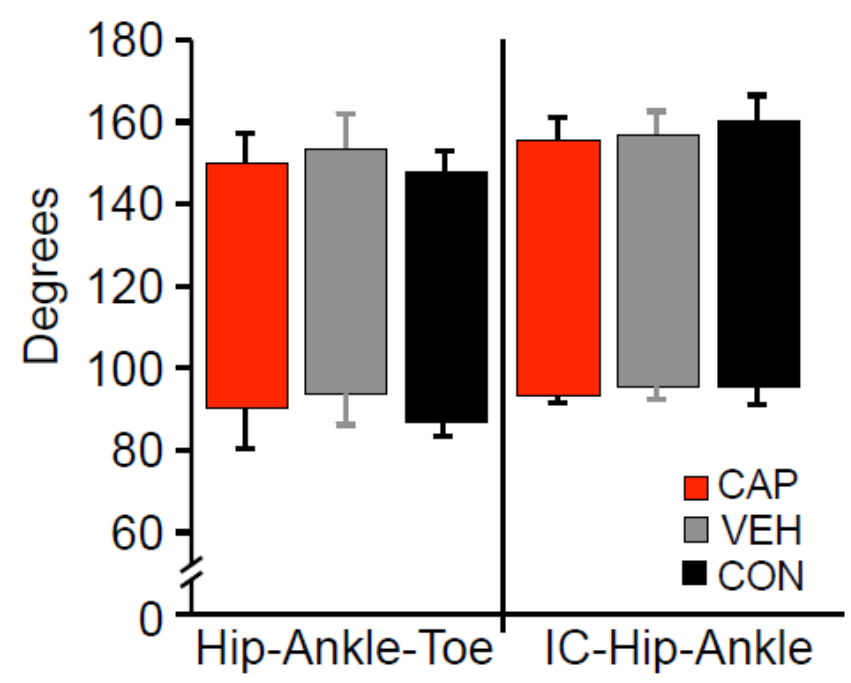

Figure 16. Baseline locomotor function of capsaicin or vehicle-treated and control (untreated) animals.

The graph shows results of 3D kinematic analysis of hindlimb angular excursions during overground stepping. The bars represent average excursions of the 2 angles (Hip-AnkleToe and Iliac Crest (IC)-Hip-Ankle) within the 3 hindlimb segments (hip, knee, ankle). Top error bars show standard deviation of the angular peaks, bottom - SD of the angular troughs. 


\section{Magnetically Evoked Muscle Potentials (MEMP)}

The CAP group had significantly smaller MEMP amplitudes (Fig. 17A) as compared to the VEH $(\mathrm{p}<.001)$ and $\mathrm{CON}(\mathrm{p}<.01)$, groups, as well as a significantly longer MEMP onset latency (Fig. 17B) as compared to the VEH $(\mathrm{p}<.05)$ group at baseline. SCI significantly reduced the MEMP amplitude $(\mathrm{p}<.001)$ and onset latency in VEH and CON animals $(\mathrm{p}<.001)$, while the responses in the CAP animals remained unchanged. After SCI all three experimental groups had MEMPs with similar characteristics. 

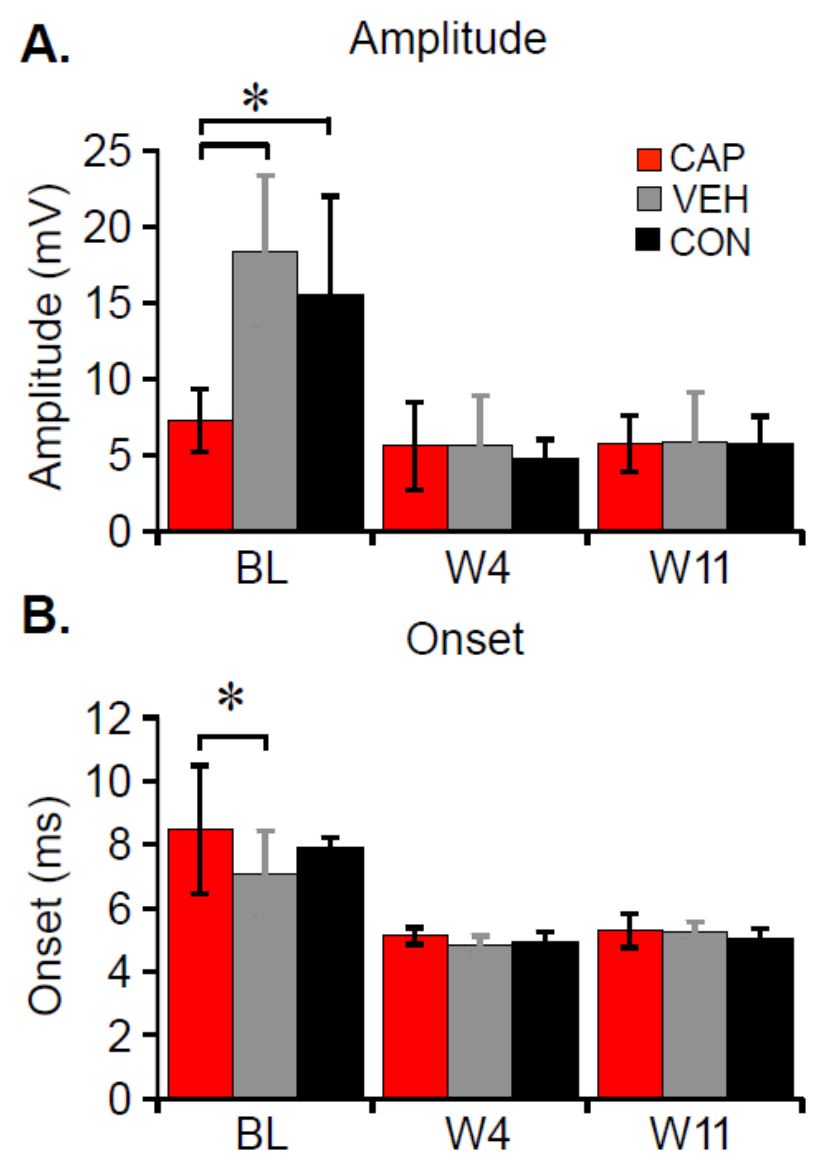

Figure 17. Characteristics of magnetically-evoked muscle potentials of capsaicin or vehicle-treated and control animals at baseline and after SCI.

The amplitude (A) and onset (B) of magnetically evoked muscle potentials (MEMP) were measured from the gastrocnemius muscle in response to magnetic stimulation at the base of the tail at baseline (BL), week 4 (W4) and 11 (W11) after SCI. Data shown as means \pm SD (RM ANOVA, Bonferroni post hoc, $\mathrm{p}<.05$ ) 


\section{Spinal cord histology.}

After spinal cord injury the CAP, VEH and CON groups had $9.4 \pm 5.3 \%, 8.4 \pm$ $4.2 \%$ and $15.5 \pm 8.69 \%$ spared white matter at the epicenter, respectively. One-way ANOVA analysis showed no significant group differences $(\mathrm{F}=2.82, \mathrm{df}=2,20, \mathrm{p}=.083)$. Locomotor function after SCI and during stretching.

Locomotor function, as assessed by BBB scores, plateaued at 3 weeks post-SCI at around 10 or 11 in all groups (Fig. 18A), although BBB subscores continued to increase slightly over the 6 weeks after injury but prior to stretching (Fig. 18B). By week 6 a significantly larger proportion of $\mathrm{VEH}$ and $\mathrm{CON}(\mathrm{p}<.05)$ animals showed consistent weight supported stepping (a BBB subscore of at least 0) as compared to the CAP group (Fig. 18B). After the first 5 days of stretching the BBB scores of VEH animals decreased significantly $(\mathrm{p}<.005)$ and were significantly lower than those of CAP animals $(\mathrm{p}=.005)$. Stretching had only minor negative effects on the locomotor function of CAP animals; their BBB scores were not significantly different after 5 days of stretching. The locomotor function of VEH animals recovered back to pre-stretch levels over the weekend, however, just one stretching session in the following week negated that recovery $(\mathrm{p}<.01$, as compared to pre-stretch values) and the next 4 days of stretching resulted in further detriments $(\mathrm{p}<.001)$. Some recovery was achieved over the second weekend without stretching, but this was not as robust as after the first week, with the BBB scores of VEH animals remaining significantly lower than those of the CAP and CON groups $(\mathrm{p}<.005)$. The same pattern of BBB score change persisted in the $3^{\text {rd }}$ week of stretching. By the end of the $3^{\text {rd }}$ week VEH animals were able to achieve BBB scores of only 2.5 . This is significantly different $(\mathrm{p}<.001)$ from their pre-stretch locomotor function when animals achieved 
consistent weight-supported stepping (BBB score of 11). On the contrary, BBB scores of the CAP rats fluctuated only slightly during the weeks of stretching (ranged from 8 to 10) and never dropped significantly below the scores of CON rats. Two weeks without stretching (weeks 9 and 10 post-SCI) allowed VEH animals to recover back to pre-stretch levels, but once stretching resumed at week 11, locomotor function again dropped to its lowest level.

Analysis of weight-supported stepping (in shallow water) is shown in Figure 18C shows the results of additional kinematic-based assessment of the animals' stepping ability with partial weight support provided by 2 inches of water in the narrow ("runway") tank during the $1^{\text {st }}$ (week 6) and $3^{\text {rd }}$ (week 8 ) weeks of stretching (both time points normalized to prestretch stepping ability at week 4). Stretching resulted in a reduced ability of both CAP (62\% of W4) and VEH ( $42 \%$ of W4) animals to step in shallow water after only 3 days of stretching at week 6 and there was no significant differences between the two groups. VEH animals had lower function than $\mathrm{CON}$ animals at week 6, the difference approaching statistical significance $(\mathrm{p}=.053)$. The stepping ability of the VEH animals was further reduced at week 8 to only (14\% of W4), while CAP animals did not have further disruption in their function, remaining around $60 \%$ of $\mathrm{W} 4$, which was significantly greater different from $\mathrm{VEH}$ rats $(\mathrm{p}<.05)$. CON animals continued to improve stepping in shallow water throughout the weeks and by week 8 had significantly greater function as compared to CAP and VEH animals $(\mathrm{p}<.01)$. 
Figure 18

A. BBB Open Field Locomotor Scale

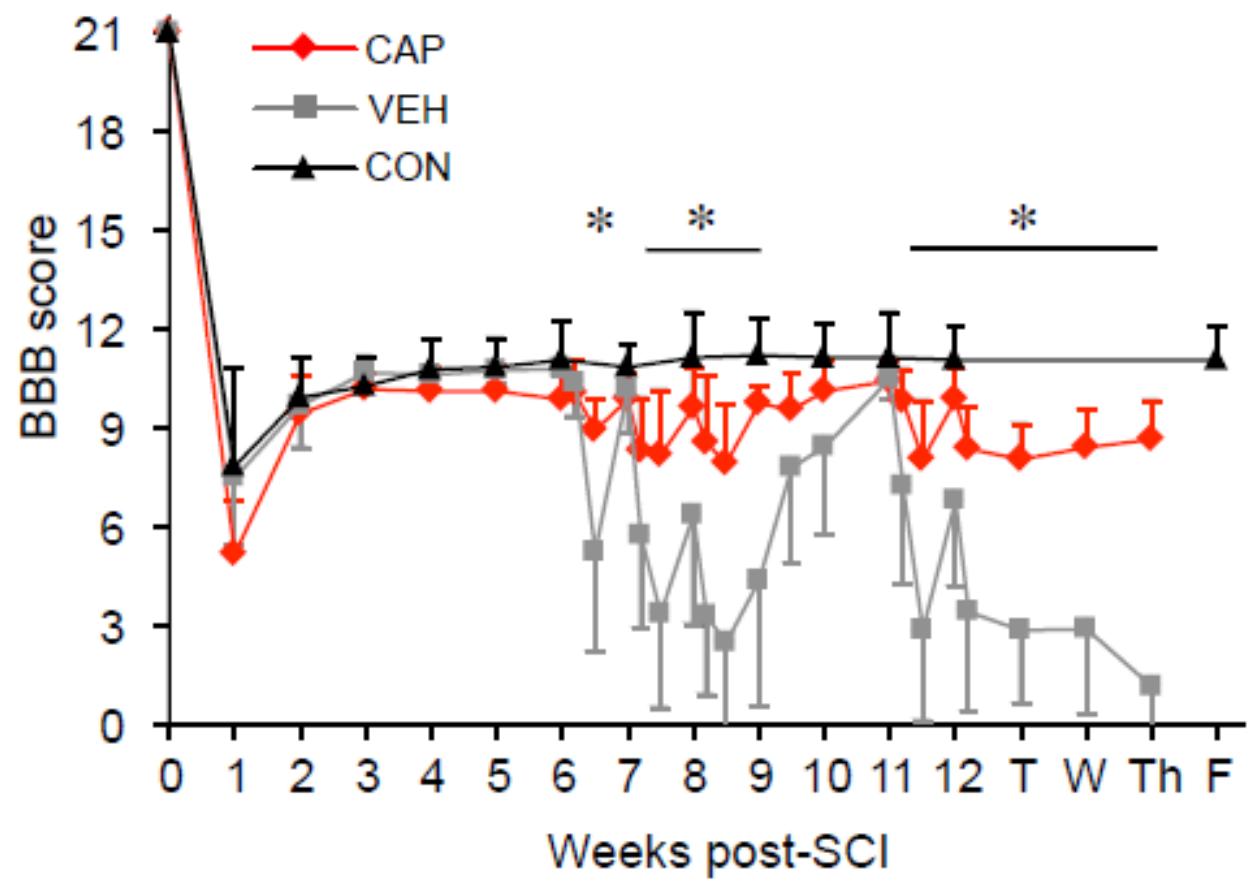

B. BBB Subscores C. Stepping in Shallow Water
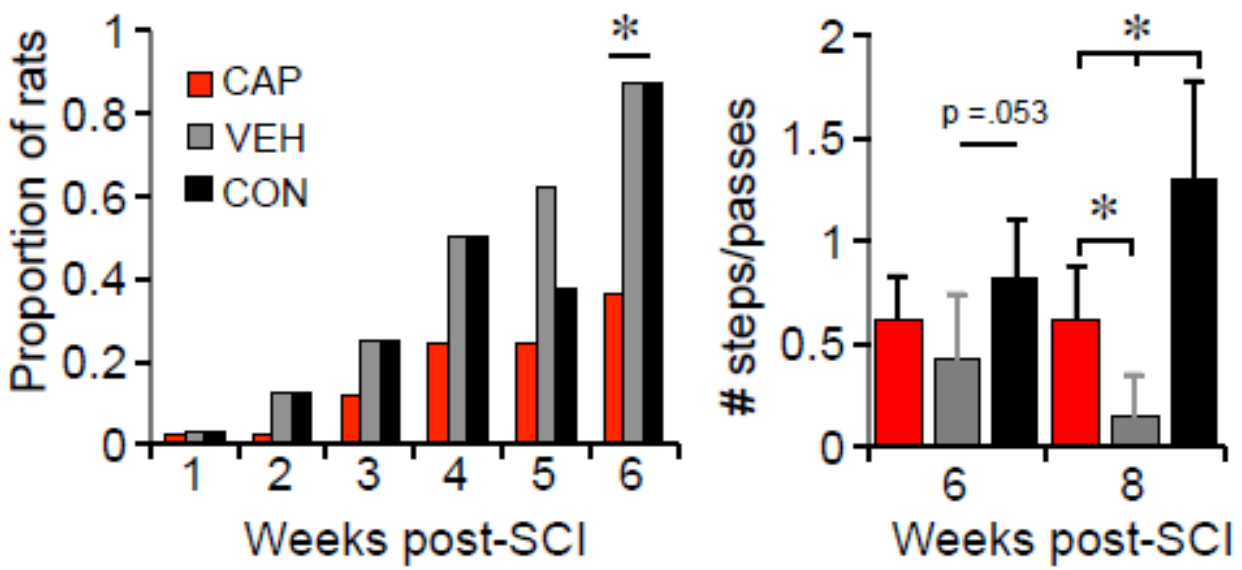
Figure 18. Locomotor function of capsaicin or vehicle-treated and control animals after SCI and during stretching.

BBB Open Field Locomotor assessment was performed weekly for the first 6 weeks after SCI (A). During the 5 weeks of the stretching protocol (beginning at 6 weeks post-SCI), BBBs were assessed three times a week (Monday am/pm and Friday pm). During week 12 the sacrificing procedures began after the post-stretch BBB assessment: T-Tuesday, $n=8$ in each group; W-Wednesday, $n=5$, Th-Thursday, $n=2$. Data shown as means \pm SD $(R M$ ANOVA, Bonferroni post hoc, $\mathrm{p}<.05)$. The proportion of animals achieving a BBB subscore (indicating the ability of consistent weight support) was analyzed using Binomial proportions test $(\mathrm{p}<.05)$. Assessment of stepping ability in shallow water (for partial body weight support) was done biweekly (C). Number of steps the animals took during each pass from weeks 6 and 8 were normalized to week 4 (pre-stretch function) for each animal (C). Data are shown as means \pm SD (RM ANOVA, Tukey HSD post hoc, $\mathrm{p}<.05)$. 


\section{CGRP analysis.}

Representative images of CGRP IHC are shown in Fig. 19. CAP animals had very low numbers of CGRP positive puncta, significantly less area than the VEH and CON groups ( $\mathrm{p}<.001)$. In addition, VEH animals (stretched, but no capsaicin-treatment) had noticeable levels of positive puncta revealed as significantly greater area of CGRP within the dorsal horn compared to CON (no capsaicin or stretching) $(\mathrm{p}<.05)$.

Activation of neurons in response to stretching.

Hindlimb stretching resulted in significant increases in the number of c-Fos positive nuclei throughout the lumbar enlargement gray matter in VEH animals as compared to the CON group, and also compared to the CAP group at L3. Figure 20 shows the mean numbers of nuclei for each lumbar segment (A) and a schematic representation of the distribution within each level for each group (B). Photomicrographs of c-Fos positive nuclei are shown in Figure 21. Figure 22 shows the c-Fos ${ }^{+}$neuron count breakdown into bilateral areas of Substantia Gelatinosa (SG), dorsal horn (DH) and intermediate gray matter and ventral horn $(\mathrm{IGM} \& \mathrm{VH})$. There were no significant differences in the number c-Fos + neurons between groups in SG except at level L5 (VEH vs CON, $\mathrm{P}<.05)(\mathrm{A})$. VEH had significantly increased number of c-Fos+ neurons in DH compared to CON $(\mathrm{p}<.01)$ at L1-L5 and at L5 when compared to CAP $(\mathrm{p}<.05)$. CAP animals had significantly greater number of c-Fos + neurons at L3 compared to CON ( $\mathrm{p}<.005)$ (B). Similarly, the number of c-Fos+ neurons of VEH rats was significantly greater as compared to CON within IGM\&VH at L1-L5 (p<.05) and at L2 $(\mathrm{p}<.05), \mathrm{L} 3(\mathrm{p}<.01)$ and L5 $(\mathrm{p}<.05)$ compared to CAP. CAP animals also had increased number of c-Fos+ neurons compared to CON at L2 (p<.01), L4 (p<.01) and L5 $(\mathrm{p}<.005)(\mathrm{C})$ 

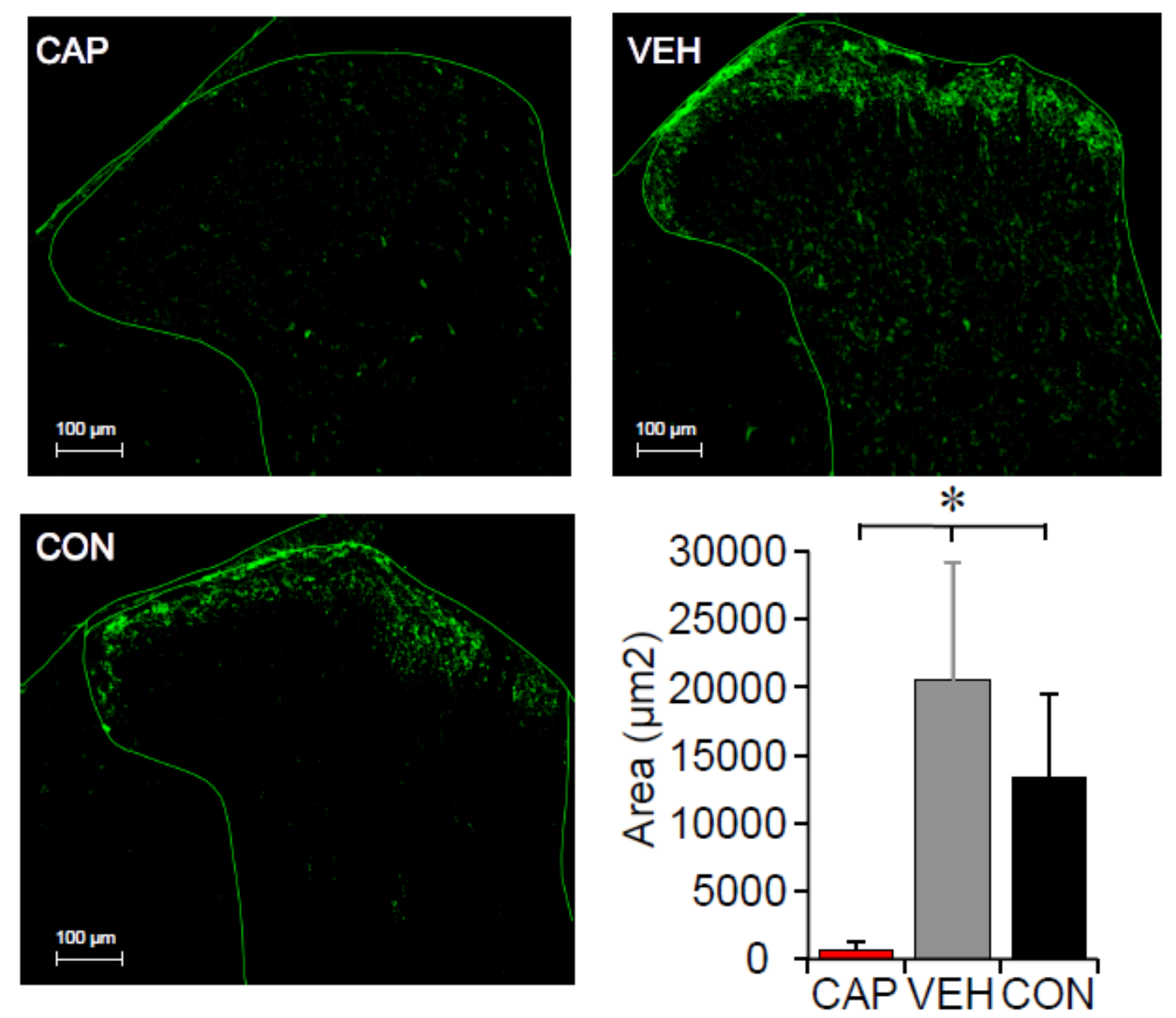

Figure 19. Images and quantification of CGRP immunoreactivity in the dorsal horn of the capsaicin or vehicle-treated and control animals.

Effectiveness of neonatal capsaicin treatment was histologically confirmed with immunohistochemistry of CGRP in the dorsal horn of the spinal cord (L3). Data shown as means $\pm \mathrm{SD}$ (One-way ANOVA, Tukey HSD post hoc, $\mathrm{p}<.05$ ) 
Figure 20
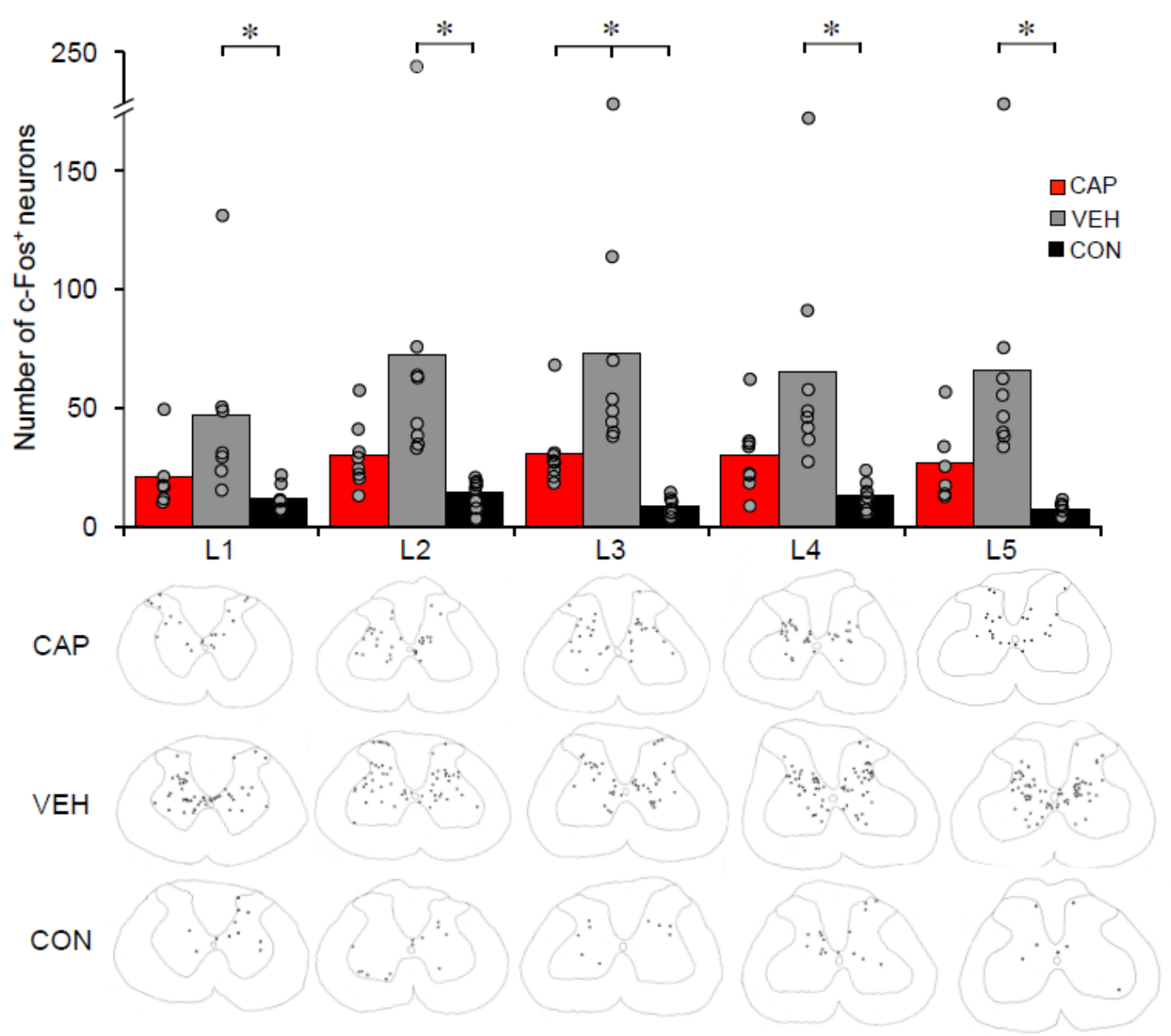

Figure 20. Quantification of the total number of c-Fos+ neurons throughout the lumbar enlargement of the capsaicin or vehicle treated and control animals and the schematic representation of their distribution. The bars represent an average number of c-Fos+ neurons from 3 sections at each level, while each dot represents individual animals (A). $\mathrm{n}=8$ per group for each level except at L1 and L5 CAP: $n=6$ and at L1 VEH \& CON $n=7$, each. Analyzed using RM ANOVA, Bonferroni HSD post hoc, $\mathrm{p}<.05$. Traces of spinal cord sections representative of each group for each level with black dots corresponds to c-Fos+ neurons within the chosen sections (B). 


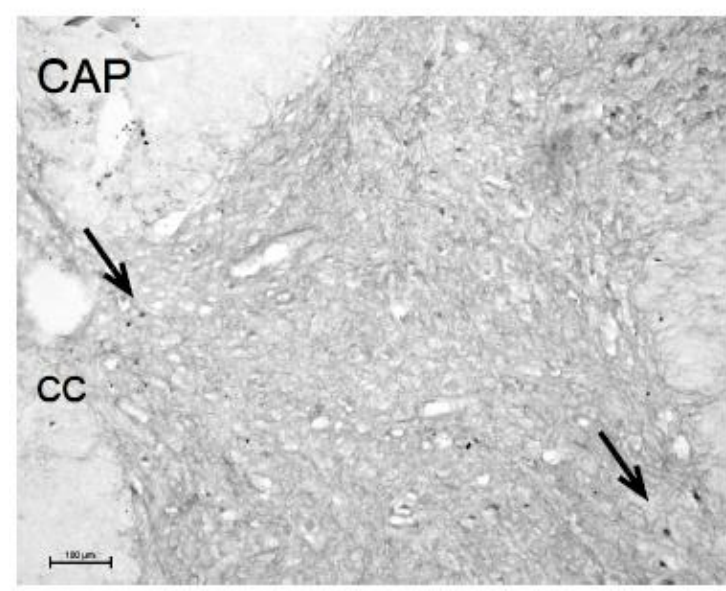

VEH (a)
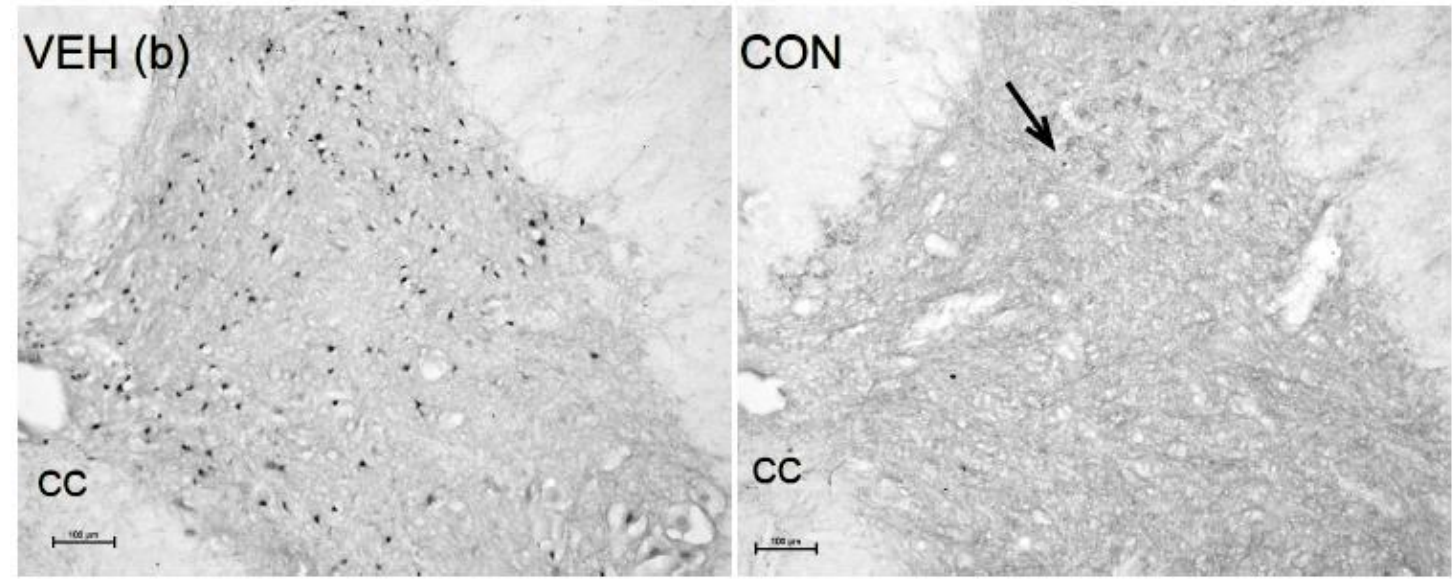

Figure 21. Images of spinal cord sections stained for c-Fos.

Arrows point to c-Fos + cell nuclei. VEH (a) is an image from an animal representative of an average number of c-Fos + neurons, sacrificed after 2 days of stretching in the last week of the stretching protocol. VEH (b) is an image from an outlier animal ( $>3 \mathrm{SD}$ away from the mean), sacrificed after 4 days of stretching that same week. CC - central canal. 
Figure 22

A.
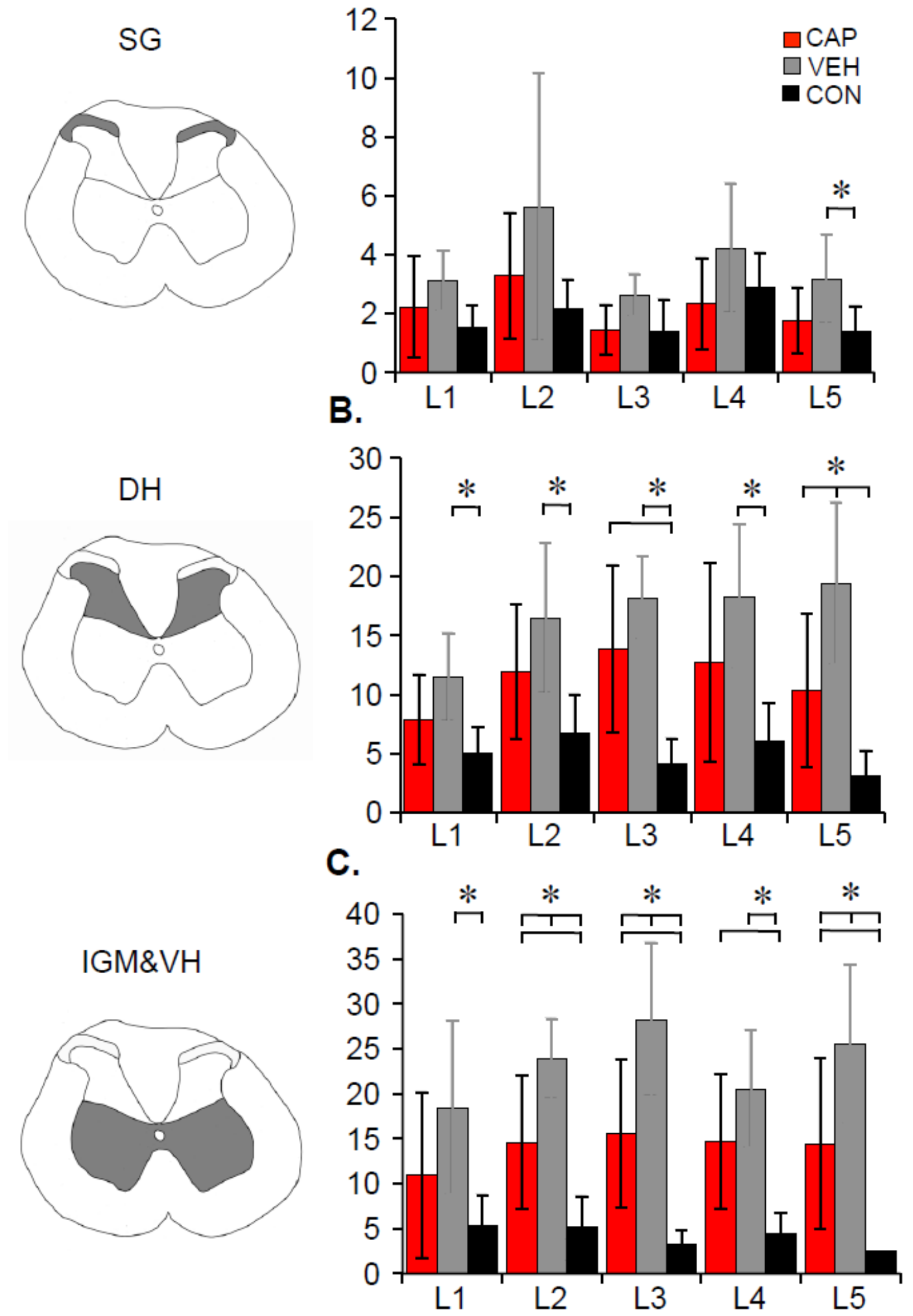
Figure 22. Number of c-Fos+ neurons separated into three general regions of gray matter.

Spinal cord regions are shown schematically next to the graphs SG- Substantia Gelatinosa (A), DH - dorsal horn (B), IGM\&VH (C) - intermediate gray matter and ventral horn (shaded areas). Data shown as means $\pm \mathrm{SD}$ (RM ANOVA, Bonferroni post hoc, $\mathrm{p}<.05$ ). Two outliers (defined as $\geq 3$ standard deviations) from VEH group were removed, so VEH $\mathrm{n}=6$ for all intraregions and levels except at $\mathrm{L} 2(\mathrm{SG}) \mathrm{n}=5 ; \mathrm{CAP}: \mathrm{n}=8$, except for $\mathrm{L} 1$ and $\mathrm{L} 5$ $\mathrm{n}=6$; CON n=8, except for L1 $\mathrm{n}=7$ and L4 (SG) $\mathrm{n}=7$. 


\section{Hindlimb responses to stretch in correlation with the number of c-Fos + neurons.}

Based on the finding that the significant differences in the number of c-Fos+ neurons came from the IGM\&VH region of the spinal cord which processes motor related information, we hypothesized that this upregulation was related to the hindlimb activation in response to stretch. To determine whether there is a relationship between the observed hindlimb responses and the number of c-Fos ${ }^{+}$neurons within IGM\&VH, an overall score for the four most common hindlimb responses (kicking, vibration, air-stepping and pull back) was generated by summing the grades of intensity/severity of the response recorded during each stretch of the last stretching session for each animal. First, we found that CAP animals had significantly higher number of kicking responses as compared to VEH $(\mathrm{p}=.001)$, whereas $\mathrm{VEH}$ rats had significantly higher number of vibration responses as compared to CAP rats $(\mathrm{p}<.01)$ (Fig. 23A). There were no significant differences in airstepping or pull back responses between the groups. In addition, we found a significant correlation between the vibration hindlimb response and the number of c-Fos + neurons at L5 for both VEH $\left(\mathrm{r}_{\mathrm{s}}=.793, \mathrm{p}=.033\right)$ and CAP group $\left(\mathrm{r}_{\mathrm{s}}=-.886, \mathrm{p}=.019\right)$ (Fig. 23B,C). Interestingly, for the VEH group it was a positive correlation whereas CAP showed a negative correlation. Furthermore, the number of c-Fos+ neurons at L1 strongly correlated with the kicking response scores in the CAP group $\left(\mathrm{r}_{\mathrm{s}}=.841, \mathrm{p}=.036\right)$ (Fig. 23D), whereas there were no significant correlations between kicking and number of c-Fos+ neurons at any level in the VEH group. 


\section{Figure 23}
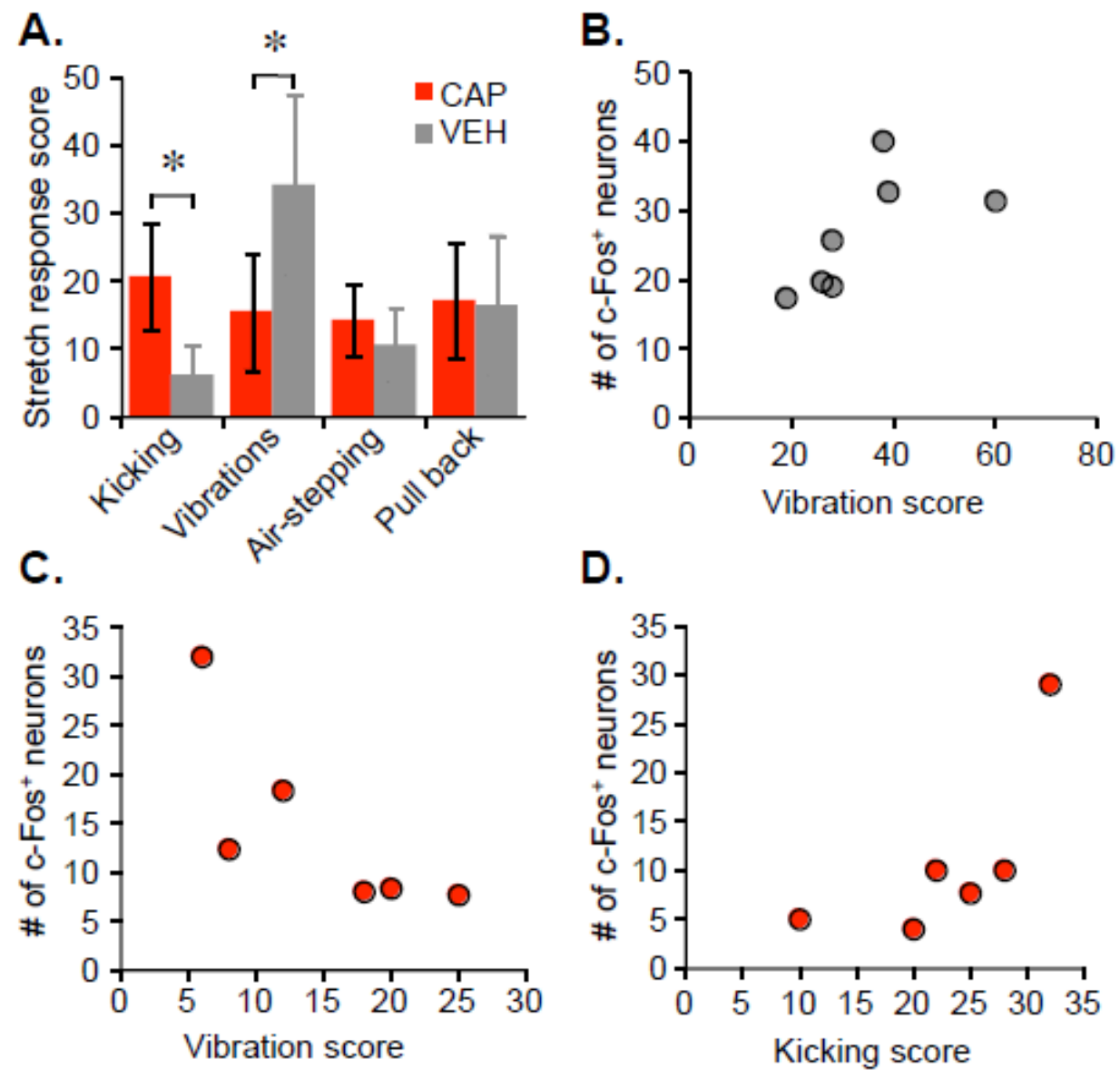

Figure 23. Quantification of the four major hindlimb responses during stretching and scatterplots of the kicking and vibration responses vs. the number of c-Fos + neurons.

The responses include kicking, vibrations, air-stepping and pull back (A); data shown as means $\pm \mathrm{SD}$, (independent t-test, $\mathrm{p}<.05$ ); (B.) VEH group vibration score has a significant positive correlation with the number of $\mathrm{c}-\mathrm{Fos}+$ neurons at L5 (rs $=.793, \mathrm{p}=.033$ ) (C.) CAP group vibration score has a significant negative correlation with the number of c-Fos+ neurons at L5 (rs =.886, $\mathrm{p}=.019)$ and a significant positive correlation between the kicking score and the number of c-Fos + neurons at L1 (rs $=.841, \mathrm{p}=.036$ ) (D.) Significant correlations were determined using Spearman's rho test. 


\section{Muscle histology.}

The proportion of muscle fibers (MF) with centralized nuclei were not different between the groups and were very close to $3 \%$, the percentage present in normal adult rat hindlimb muscles (A). VEH rats had significantly reduced MF cross sectional area (CSA) in the Tibialis Anterior muscle as compared to CON and CAP rats $(\mathrm{p}<.05)$. CAP rats had larger MF CSA in the Medial Gastrocnemius as compared to VEH (p<.05) (B). 


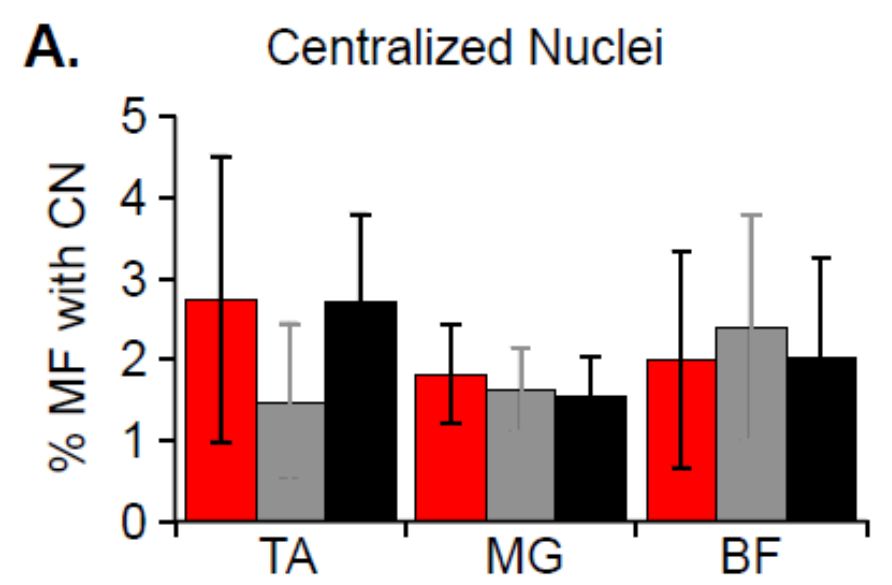

B. Cross Sectional Area

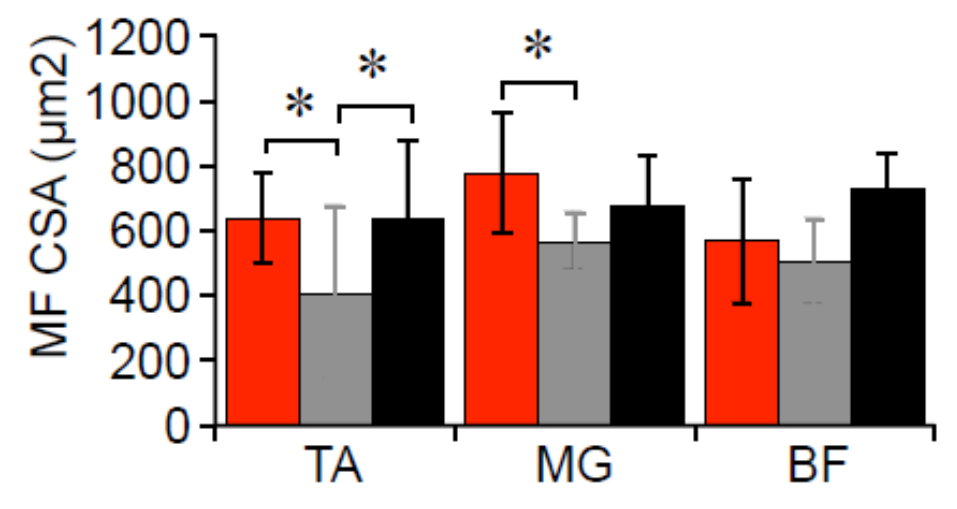

Figure 24. Hindlimb muscle analysis for presence of centralized nuclei and muscle fiber cross sectional area.

Tibialis Anterior (TA), Medial Gastrocnemius (MG), Biceps Femoris (BF) were analyzed for number of muscle fibers (MF) with centralized nuclei ( $\mathrm{CN}$, marker of regeneration) (A) and muscle fiber cross sectional area (CSA) (B). Data shown as means \pm SD (Oneway ANOVA, Tukey HSD post hoc, $\mathrm{p}<.05$ ) 


\section{Discussion}

Functional aspects of capsaicin treated animals pre-and post-SCI.

To our knowledge, this is the first report of a neonatal capsaicin-treated animal model of adult SCI. In order to confirm the success of C-fiber depletion by capsaicin treatment the animals were tested for thermal and mechanical thresholds to nociceptive stimuli prior to SCI. Consistent with previous findings [272], our CAP animals had intact mechanical nociception but significantly increased thermal nociceptive thresholds (Fig. 1). Reduction of nociceptive afferents had no influence on baseline locomotor function. Hindlimb joint excursions assessed using 3D kinematics and gait were not different from nociceptor-intact rats. However, lack of nociceptive afferents did subtly influence locomotor recovery after SCI. At 6 weeks post-injury the proportion of CAP animals capable of consistent weight supported stepping (subscore of 0) was significantly lower than the VEH and CON groups despite BBB scores being similar. Epicenter spared white matter was also similar therefore injury severity could not account for the group differences in weight bearing stepping. Stimulation of nociceptive muscle afferents can modulate an ongoing fictive locomotor rhythm [241], and our findings hint at their potential contribution to recovery of weight supported stepping after spinal cord injury. Certainly, this finding is relevant to our overall understanding of the role of afferents in locomotor function after SCI and will need further investigation. An additional observation of interest is that nociceptor-depleted animals never recovered bladder function after SCI and required daily manual expression for the duration of the experiment. Zinck et al., showed that sprouting of lumbosacral CGRP positive primary afferents precedes reemergence of bladder function after complete spinal cord transection [273]. We confirmed that CAP animals had dramatically reduced CGRP signal in the dorsal horn of the L3 segment. These 
observations strongly support the notion that CGRP-expressing afferents, and possibly their spouting is required for the reemergence of bladder activity post-SCI.

\section{Stretching and locomotor function.}

Stretching initiated at 6 weeks post-SCI resulted in a significant disruption of locomotor function in nociceptor-intact (VEH) rats, consistent with our previous observations. Also in keeping with our previous study [218] the first stretching session did not have a significant negative effect on locomotion. Daily stretching, however, had a cumulative effect; the post-stretch locomotor assessment on day 5 revealed significant impairment. The animals experienced significant recovery over the weekend following the first week of stretching, but one stretching session on the Monday of the second week dramatically reduced locomotor function. After three weeks of daily stretching locomotor function of the VEH rats dropped from the pre-stretch BBB score of 11 to around 2.5, even lower than their scores recorded at 1 week post-SCI. Two weeks without stretching allowed the animals to return to pre-stretch locomotor function, indicating that stretch-induced locomotor deficits are temporary, in agreement with our previous observation. However, after two weeks off, re-initiating stretching induced a dramatic drop in locomotor function following a single stretching session. Overall, the pattern of locomotor disruption and recovery over time suggests that daily stretching sensitizes or primes the system and leads to the large functional declines.

Stretching had only a minor effect on locomotor function of nociceptor-depleted (CAP) rats. Throughout the weeks of stretching this group maintained, at a minimum, extensive movement in all three joints (an average BBB score of 7.5). The most noticeable effect of stretching on the locomotor function of CAP animals was during shallow water 
stepping. At weeks 6 and 8 (during the stretching protocol) CAP rats generated $40 \%$ fewer steps (per pass) as compared to week 4 which most likely resulted from a prolonged step cycle. The animals tended to keep their hindlimbs extended and dragging for longer periods of time when provided with the body weight support of buoyancy. Thus, instead of taking 3-4 steps per pass the animals took only 2 steps while making most of the advancements using forelimbs. This affect could potentially be mediated by the remaining nociceptive afferents since neonatal capsaicin treatment is never complete. Alternatively, this might result from a nociceptor-independent mechanism such as stretching-induced muscle spindle insensitivity, suggested previously to explain reductions in H-reflex after prolonged stretching [224]. It is well-established that activation of locomotor generating spinal circuitry is heavily dependent on proprioceptive afferent input after SCI [121]. Nevertheless, all eight of the CAP rats maintained the ability to step through the weeks of stretching, while VEH rats had substantial reductions in their ability to step such that by week 8 only 2 rats could produce some steps and in only one hindlimb.

It is interesting to note that while stretching is a mechanical stimulus, depletion of nociceptive afferents known primarily as "thermally responsive" (TRPV1 positive neurons) had the effect of protecting locomotor circuitry from stretch-induced disruption. In a previous study we discussed the possibility that eccentric muscle contractions during stretching may be key initiating factor leading to impaired locomotion. We reported that stretching evokes air-stepping and clonus-like vibrations in both the contralateral (unstretched) and ipsilateral (stretched) limbs. Eccentric contractions are very effective at inducing delayed onset muscle soreness (DOMS)[274], a phenomenon dependent on the sensitization of TRPV1 positive neurons [275]. We saw kicking and other motor responses 
in the current experiment which likely would have resulted in eccentric muscle contractions during stretching and likely subsequent sensitization of nociceptive afferents in VEH rats. Importantly, rats treated with capsaicin as neonates are known to be resistant to developing DOMS after eccentric muscle contractions [276]. Whether or not eccentric contractions/DOMS are necessary and/or sufficient for stretch-induced locomotor deficits after SCI is currently unknown, but it is feasible that similar physiological mechanisms underlie both DOMS and the stretching phenomenon.

It is highly likely that the stretching employed in our protocol activates Golgi tendon organs (GTOs), however, we do not expect that autogenic inhibition played a role in any stretch-induced reduction in the locomotor capacity for a few reasons. First, the inhibitory effect on the homonymous motoneurons produced by GTO signaling does not persist after the stimulus is removed, in fact, the inhibitory effects diminish with an ongoing muscle contraction [277]. In addition, GTOs have been shown to modulate an ongoing muscle activity by providing excitatory feedback [278]. Furthermore, SCI may result in impaired autogenic inhibition and at least in humans the functional deficits in this circuitry have been proposed to contribute to the development of spasticity after neurotrauma [279]. Histological findings.

CGRP immunoreactivity was essentially negligible in lumbar spinal cord sections from CAP animals. This confirmed the effective depletion of many CGRP positive (TRPV1 positive) afferents. However, we discovered that VEH rats (nociceptor intact) showed increases in CGRP area in the dorsal horn when compared to CON (unstretched injured) animals. This finding is consistent with the functional data, specifically the involvement of nociceptive afferents in stretch-induced locomotor deficits and the increase 
in sensitivity of the locomotor circuitry to the negative effects of stretching over time. It is well documented that certain kinds of repetitive activation of nociceptive afferents leads to their central and/or peripheral sensitization[280]. Moreover, plasticity within the nociceptive circuitry after SCI has been implicated in neuropathic pain [281] and autonomic dysreflexia [282] as commonly seen in SCI patients. We speculate that both the activation of nociceptive afferents during stretching and the lack of hindlimb activity following stretching contributes to the increased CGRP within the spinal cords of the VEH animals [283].

Using c-Fos as a marker for neuronal activation we determined that VEH (nociceptor intact, stretched) animals had significantly higher numbers of c-Fos + nuclei, compared to $\mathrm{CON}$ animals, throughout the lumbar enlargement. In contrast, CAP (nociceptor depleted) animals had similar numbers of c-Fos+ neurons to both VEH and CON groups, except at L3 where they had significantly more c-Fos + nuclei than the CON group. All the animals were sacrificed 2 hours after the last stretching session, however simply due to scheduling and manpower some animals were sacrificed after 2, 3 or 4 consecutive days of stretching. As a result we discovered that VEH animals sacrificed after 4 days of stretching had much higher numbers of c-Fos ${ }^{+}$nuclei when compared to those sacrificed after 2 or 3 days of stretching (Figures 6 and 7). We then divided the gray matter counts intro three general areas (Fig. 8) and found that VEH animals had significantly higher numbers of $\mathrm{c}-\mathrm{Fos}^{+}$nuclei in the intermediate gray matter and ventral horn as compared to CAP and CON rats. This finding suggests that afferents activated by stretching induce c-Fos expression in spinal cord interneurons throughout the lumbar gray matter and specifically in the intermediate gray matter, a region known to contain interneurons 
responsible for integrating and generating motor output. Since we indeed observe a plethora of motor responses during stretching and have always kept a thorough record specifying the frequency/intensity of each response we were able to generate a "stretch response score", compare the scores for each response between groups and determine whether there is a relationship between the hindlimb responses and the number of c-Fos ${ }^{+}$ neurons (Fig. 9). Interestingly, the two major hindlimb responses - kicking and vibrations - had a different, essentially opposite from one another, profile of expression between the groups. The vibration response, which we previously described in detail as having features close to those of a human clonus, was the most robust response in the VEH group and thus it is tempting to speculate that activation of nociceptive afferents greatly contributes to its observation. If in fact vibration we see in rats is functionally similar to the human clonus, then this observation also potentially implies a novel mechanisms for triggering of clonus - nociceptive afferent activation. Interestingly, in a series of human case studies on severe spinal myoclonus in SCI subjects, a subtle link between an existing painful condition and observation of clonus was established. After the resolution of a musculoskeletal pathology clonus could no longer be evoked in those SCI patients [55]. Recently, a robust nocifensive kicking behavior in response to painful mechanical paw stimulus was described in the rat referencing its similarity to clonus [261]. Description of the kicking response in the study by von Gorp is similar to the kicking we observe during stretching. It is possible that the robust kicking response in the CAP rats resulted from activation of other types of nociceptive afferents responsible for mechanonociception, for example. Thus, both vibration and kicking responses could be equivalent to human clonus even though they 
have some differences in appearance, presumably attributable to the type of afferent activation that drives that response.

Significant positive correlations were identified between the vibration scores and the number of c-Fos + neurons at L5 in VEH rats (Fig. 9B) as well as kicking scores and the c-Fos + neurons at L1 in CAP rats, (Fig. 9D) suggesting that motor responses during stretching result in upregulation of c-Fos in the IGM\&VH of the spinal cord. Ankle muscles which visually have the greatest activation during the vibration are innervated by motoneurons at L5, whereas L1 contains motoneurons innervating the hip musculature that visually appear to be the "driving" force behind the kicking response. It is possible that nociceptive signaling leads to increased excitability of the motor system that in long-term may have detrimental consequences, such as temporary loss of locomotor function in the stretched rats and when it occurs in uncontrollable fashion it leads to the disruption of spinal learning and locomotor function as has been clearly demonstrated in studies by Grau et al [250].

We have determined that present of nociceptive afferents is required for the observation of the stretch-induced locomotor deficits which occur in the absence of concurrent muscle tissue damage. Stretched limbs showed no increase in the number of regenerating muscle fibers. Some atrophy, presumably due to disuse, occurred in the Tibialis Anterior muscle of the VEH rats.

In conclusion, the findings of this study suggest that TRPV $1^{+}$nociceptive afferents may contribute to locomotor recovery after SCI, however, their presence is also required for the stretching to have its negative effect on locomotor function that we have consistently observed. While the first observation could be explained by a complex 
modulatory effect of nociceptive afferents on locomotor circuitry through the flexor reflex afferent pathway [241], the latter effect might be the result of maladaptive plasticity resulting from "uncontrollable" nociceptive signaling in the already vulnerable post-injury spinal cord exhibiting a compromised inhibitory system [172]. While the clinical relevance of the stretching phenomenon still needs to be established, these findings nonetheless have significant implications for rehabilitation after SCI. Harvey et al., demonstrated that some physical therapists apply torques when stretching "sensory complete" SCI patients of sufficient magnitude to activate nociceptive afferents and would thus be intolerable to sensate individuals [211]. Furthermore, in the field of sports performance it has been documented that static stretching impairs certain aspects of motor output [284]. These minor effects observed in the spinal-intact population might have more severe consequences in SCI patients. Given the general ineffectiveness of stretching for its intended purposes after SCI [98], reports of its negative effects on performance in athletes and our data showing that stretching disrupts locomotor function after SCI in rats, the place of stretching as routine therapy in rehabilitation after SCI needs to be reevaluated. 


\section{CHAPTER VII}

\section{GENERAL DISCUSSION}

\section{Summary of the Main Findings}

In summary, we found that both static and dynamic hindlimb stretching protocols are detrimental to the locomotor function of rats with acute or chronic, mild or moderately-severe SCI without overt muscle damage. It was also determined that stretchinduced locomotor deficits are dependent on the activation of nociceptive afferents that occurs with torques well within those reported during stretching of patients with SCI when compared strictly based on the body weight ratio. It appears that nociceptive afferent signaling initially activates locomotor circuitry that can be observed behaviorally as hindlimb kicking or clonic-like muscle contractions in response to stretch. This activation results in upregulation of c-Fos in the neurons within the dorsal horn as well intermediate gray matter and ventral horn of the spinal cord. In addition, stretching results in an increased CGRP area in the dorsal horn. Schematic representation of the main findings of the current thesis is depicted in Figure 25. 


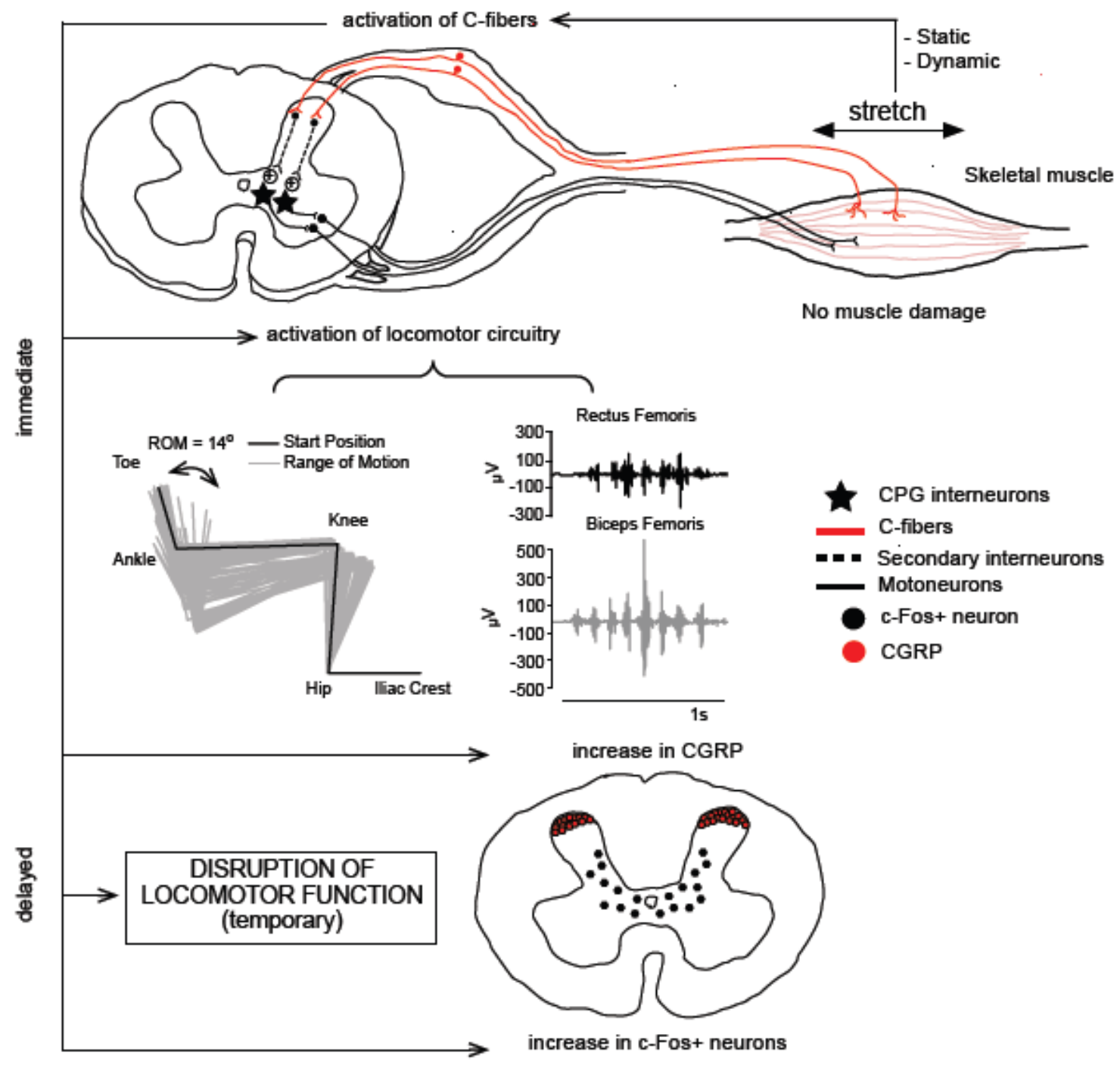

Figure 25. Summary of the main findings.

Stretching activates nociceptive afferents that have an excitatory effect on locomotor circuitry likely via indirect projections. Functionally, this activation manifests as contralateral hindlimb movements in response to stretch, including kicking, clonic-like vibrations and spasms.. Presumably as a result of this activity there is an increase in the number of c-Fos positive neurons predominantly in the intermediate gray matter (laminae VII \& X) as well as in the dorsal and ventral horns. In addition, there is an increase in the CGRP area in the dorsal horn suggesting that nociceptive afferents undergo plasticity as a result of stretching following spinal cord injury. 


\section{Nociceptive afferents modulate locomotor CPG}

The majority of innervation to the skeletal muscle is composed of small diameter, mostly nociceptive afferents [264, 265]. These high threshold thinly myelinated (A $\delta$ /group III) or unmyelinated sensory (C fibers/group IV) afferents become activated by stimuli that are damaging or potentially damaging to the tissue. The central projections of nociceptive afferents terminate within the superficial lamina of the dorsal horn within the spinal cord where they synapse onto the secondary neurons: nociceptor specific and wide dynamic range neurons. They excite interneurons that project to supraspinal centers via the spinothalamic tract where the signals are processed and interpreted as pain. Nociceptive afferents also directly synapse onto motoneurons and mediate the withdrawal reflex and via other neurons, the cross extensor reflexes [285]. Besides the reflexive control of motor output nociceptive afferents have been implicated in the modulation of more complex neuronal functions, like locomotion. It has been shown that activation of nociceptive afferents from muscle in the cat can modulate an ongoing fictive locomotor rhythm by accentuating the transition from active to non-active phases of locomotor-like bursts or override the rhythm inducing a transient tonic hyperactivity [241]. Application of noxious heat to the foot pad of the decerebrate cat facilitates locomotor-like activity [286]. Similar observations of nociceptive afferent mediated increases in locomotor-like bursts were reported in isolated preparations of mouse spinal cords [287]. Furthemore, Lev-Tov et al., have determined 
that stimulation of high threshold sacrocaudle afferents (SCA) in the neonatal spinal cord preparation evokes locomotor-like bursting in the lumbar region [288, 289]. The anatomical and electrophysiological experiments provide evidence for the existence of sacral interneurons that project to lumbar CPG through the dorsolateral and ventral funiculi. The findings from another group highlighted specific contributions of TRPV1 positive afferents in the SCA-induced locomotor rhythm while also showing that application of capsaicin to the thoracolumbar region resulted in uncoordinated hyperactivity in the first 5 minutes and complete blockage of electrically-evoked discharges (from sacral root 4 (S4) stimulation) after 10 minutes of continual application [290, 291]. Furthermore, Mandadi et al., demonstrated that TRPV1+ afferents entering through the S4 root have direct multisegmental projections including L2 and L5 in the dorsal column and Lissauers tract. The authors suggests that at the rostral levels these afferents project to the dorsal interneurons that have connections to the locomotor CPG neurons and, therefore, indirectly influence the locomotor rhythm [290]. Although these the Mandadi observations were made from the sacral nerve (S4) that does not innervate hindlimbs, Kniffki showed that the stimulation of group III and IV afferents in the gastrocnemius and soleus muscles was able to induce transient periodic activity invarious motoneuron pools [241]. We determined that the negative effects of stretching on locomotor function of the SCI rats are dependent on nociceptive afferent activation. The activation of nociceptive afferents leads to the increase in c-Fos ${ }^{+}$neurons in the intermediate gray matter (especially lamina X) and ventral horn, the areas that are involved in the generation of motor output. One of the typical hindlimb responses we observe during stretching is kicking that occurs at clonic-like frequencies. In a study by van Gorp et al., 
the authors reported hindlimb kicking in response to a stimulus which was known to be nociceptive and their description sounds very similar to what we observe during stretching [261]. Thus the hindlimb activity we observe during hindlimb stretching appears to be evoked by the nociceptive afferents.

\section{Possible mechanisms of nociceptive afferent dependent stretch-induced locomotor deficits in rats with $S C I$}

The question then is: if the nociceptive afferents influence spinal motor centers and possibly coordination centers, then how does the stretching result in disruption of locomotor function? Based on our observation of the upregulation of c-Fos, a marker for a novel neuronal activity, in the region of the CPG neurons of the stretched rats and significantly lower number of c-Fos+ neurons in the control SCI animals capable of consistent weight supported stepping, it appears that modulation of motor circuits by nociceptive afferents is different than the typical activation of the CPG during locomotion Further cellular mechanisms will need to be elucidated, however, some speculations could be made based on the extensive work by Grau et al., who have demonstrated the disruptive effects of uncontrollable nociceptive stimulation on spinal learning and locomotor recovery after SCI [172, 212, 213, 250]. The cellular mechanisms discussed below were determined using an instrumental learning paradigm. Animals with complete spinal transection are paired with one placed into each of a master and yoked group. The master rats receive an electric shock to the Tibialis Anterior when the limb is not in a flexed position. Over a 30 minute stimulus session the master rats learn to maintain the hindlimb in a flexed position and thus avoid the shock exposure (spinal training/controllable stimulation). Because the neural circuitry involved is distal to a spinal cord transection, the mechanisms are understood to reside in the spinal cord (and perhaps some in the muscle). Yoked rats receive 
the same stimulus with the same timing as the master rats regardless of the limb position (uncontrollable stimulation). The animals are later tested by assessing the duration of the flexion response to each shock over a 30 minute period. The master rats rapidly (within 4 shocks) show flexion durations of close to 1 minute as compared to naïve (not stimulated previously) rats which learn slower, but do acquire similar flexion response durations by the end of the testing session (which can be considered a "first-training-session" for those rats), while yoked rats fail to show a flexion response. The important point to make here is that prior exposure of the yoked rats to the uncontrollable nociceptive stimulation disrupts the capacity for spinal learning for at least 48 hours.

Instrumental spinal learning results in the upregulation of several pro-plasticity markers such as brain-derived neurotrophic factor (BDNF), calcium/calmodulin activated kinase II (CaMKII), cAMP response element binding protein (CREB) etc. [292]. Uncontrollable nociceptive stimulation also engages spinal circuitry resulting in activitydependent plasticity, however, it is maladaptive in nature as it results in the generalized state of neuronal hyperexcitability which prevents adaptive learning [213]. If this overall hypothesis applies to our observations of nociceptive afferent-dependent negative effect of stretching on locomotor function, then we can further suggest that the engagement of nociceptive afferents in an uncontrolled fashion also disrupts the adaptive plasticity that has occurred. In fact the animals with chronic severe SCIs that have plateaued in their locomotor function for several weeks are especially vulnerable to the nociceptive inputs as the most severe negative effects of the stretching protocol were observed in these rats. Specific cellular mechanisms involved in the maladaptive plasticity as determined by Grau et al., are dependent on post-synaptic activation of group I metabotropic glutamate receptor 
(mGluR) as well as substance P receptor neurokinin 1 (NK1R) which leads to an increased intracellular calcium, activation of protein kinase C (PKC), CamKII and phosphoinositide 3-kinase (PI3K) [293-295]. It is proposed that the activity of calcium-dependent kinases leads to an altered NMDA and AMPA receptor function [296, 297]. Particularly, nociceptive signaling induces trafficking of AMPA receptors that lack the GluA2 subunit to the cell membrane. Since AMPA receptors GluA2 subunits are essentially calcium ionophores their upregulation further increases intracellular calcium initiating a positive feedback loop that leads to an altered cellular function. [213, 298]. Nociceptive signaling also activates glial cells which have been shown to mediate long term potentiation (LTP) in the spinal cord and specifically have an important role in inducing central sensitization of nociceptive afferents [299]. The glial cell products and mediators of these effects include nitric oxide, prostaglandins, cytokines IL-1b and IL-6 as well as tumor necrosis factor alpha $(\mathrm{TNF} \alpha)[300]$. Huie et al., have shown that TNF $\alpha$ plays a critical role in the induction of spinal learning deficits [298] likely through the mechanisms involving AMPA receptor trafficking as has been demonstrated by others [301]. In addition Grau et al., has investigated the role of endogenous opioids in the mediation of spinal learning deficits as a result of uncontrollable nociceptive stimulation. They specifically found that kappa receptor (KOR) antagonist given immediately prior to the testing attenuates the learning impairments induced by uncontrollable stimulation but its administration a day prior to stimulation does not have a preventative effect [302] suggesting that KOR ligand mediates expression but not the induction of the learning impairment [250]. This observation, as suggested by Grau et al, demonstrates that NMDA-dependent adaptive plasticity remains functional since the spinal learning impairment was reversed with an opioid antagonist 
treatment and furthermore implies that this phenomenon is an example of metaplasticity as described by W. Abraham [250, 303]. In our experiments $4-5$ weeks of stretching resulted only in transient locomotor deficits as the animals' recovered locomotor function within 2 weeks of cessation of daily stretching $[218,254]$. However, when the rats are stretched for 8 weeks locomotor recovery is limited and some deficits in the finer aspects of locomotion persist $[165,171]$. This suggests that initially (for the first 5 weeks) stretching impairments appear to be metaplastic in nature, however, if carried out for longer periods some permanent plasticity takes place that might limit the capacity for adaptive learning.

\section{Future directions 1: deciphering physiological mechanisms}

The studies by Grau et al., have greatly contributed to our understanding of spinal cord learning in the absence of supraspinal input and the mechanisms behind the nociceptive-dependent maladaptive plasticity that disrupts learning. Thus, in our future experiments we can use similar pharmacological agents to determine if the stretch-induced locomotor deficits involve comparable mechanisms at the cellular level. Perhaps one of the most straight forward questions for the investigation in the next stretching experiment is if, and to what extent the negative effect of stretching is dependent on endogenous opioid peptides. This question is not only prompted by the observations made by Grau et al., as other investigators have also reported negative effects of opioids on locomotor-like activity [304, 305]. Most recently Hook et al., found that administration of morphine, an opioid receptor agonist resulted in reduced sensory and motor recovery after SCI [306-308]. In the intact CNS the effect of transmission of impulses from nociceptive afferents is under the control of supraspinal systems originating in the midbrain (periaqueductal grey), and medulla (reticularis gigantocellularis (RG) and nucleus raphe magnus, (NRM))[309]. Descending serotonergic fibers from RG and NRM synapse onto the opioidergic neurons 
in the spinal cord. Opioid peptides inhibit nociceptive transmission at the terminal of these primary afferents and also at the level of secondary dorsal horn neurons that send ascending projections in the spinothalamic tract [285]. Out of thirty known opioid peptides, the three major types represented in the spinal cord are the enkephalins (met and leu, present in lamina I-III and $\mathrm{V}$ of the dorsal horn), $\beta$-endorphin (ventral horn and lamina $\mathrm{X}$ ) and dynorphin (lamina I-II, V ) [310]. Interestingly, dynorphin is also found in the terminals and the cell bodies of peptidergic $\mathrm{C}$-fibers $\left(\mathrm{CGRP}^{+}\right.$and substance $\left.\mathrm{P}^{+}\right)$although in smaller amounts as compared to the dorsal horn neurons [310]. A few studies have shown that dorsal rhizotomy results in partial (1/3) depletion of dynorphin signal in the dorsal horn of the spinal cord $[311,312]$. Upregulation of dynorphin is seen in several pathological conditions involving inflammatory [313] and neuropathic [314, 315] states as well as SCI $[316,317]$. There is evidence to support the notion that this increase occurs in the primary afferent fibers as well [318]. However, unlike enkephalin or $\beta$-endorphin, dynorphin has little or no antinociceptive action [319]. On the contrary, its elevation has a crucial role in the maintenance of pronociceptive state in the spinal cord $[305,320]$. Several studies demonstrated that dynorphin has, in addition to its opioid receptor mediated effects, actions through non-opiate mechanisms which can explain this paradoxical phenomenon [318, 319] Furthermore, Faden et al., has shown that intrathecal administration of dynorphin leads to an increased secondary tissue damage following SCI and subsequent reduction in motor function, whereas administration of dynorphin antagonist has a tissue sparing effect and improves locomotor outcomes after SCI [305, 321]. Dynorphin has the highest affinity for kappa-opioid receptor [285] which is implicated in mediation of spinal learning deficits [302]. In another study, administration of naloxone, that blocks all opioid receptors, to 
chronic spinal cats induced rhythmic locomotor-like movements in the hindlimbs and an onset of bladder contractions [322], suggesting that the motor and autonomic circuitry after a severe SCI might be suppressed by the presence of endogenous opioids and dynorphin specifically considering the evidence from all other studies. Early in-cage activity and robust locomotor recovery in rats which is not seen in untrained cats with full transections [164] might keep maladaptive plasticity within the opioidergic system from occurring, however, stimulation of nociceptive afferents as occurs in stretching might induce release of endogenous opioid peptide that depresses the locomotor CPG. We hypothesize that administration of opioid antagonist prior to locomotor assessments will result in "unmasking" of locomotor function from stretch-induced negative effects if in fact the underlying mechanisms are similar to those seen in disruption of spinal learning.

\section{Consideration of the physiological role of group III and IV afferents after SCI}

Group III and IV afferents have a well-established physiological role in the maintenance of muscle homeostasis during exercise in healthy humans [206]. At the onset of muscle contraction activation of these afferents leads to an increase in cardiopulmonary responses during exercise through their modulation of cardiac and respiratory centers (nucleus tractus solitaries) in the ventrolateral medulla[323]. The continuous feedback from these muscle afferents is crucial for preventing premature fatigue as it has been shown that inhibition of group III and IV afferents (specifically non-nociceptive type, so called "ergoreceptors") during exercise in humans significantly compromised cardiac output, mean arterial blood pressure, femoral artery blood flow as well as pulmonary ventilation which ultimately resulted in arterial hypoxemia and metabolic acidosis [207, 324]. On the

other hand, group III/IV afferents have inhibitory effects on spinal motoneurons during intense exercise and with prolonged fatiguing exercise they also inhibit corticospinal drive, 
a phenomenon known as central fatigue (reduction of motor output) [325]. In addition, capsaicin-sensitive afferents have an inhibitory effect on the monosynaptic reflex [326] which constitutes a substantial component of total neural drive to the muscle during walking. For the human soleus muscle, primary afferent feedback has been estimated to account for $30-60 \%$ of motoneuronal excitability [327]. What happens to this system after SCI? This question has not been addressed perhaps because most SCI patients are wheelchair dependent and have a significantly diminished level of physical activity. However, with the recent progress in our understanding of the potential of the spinal cord to undergo adaptive plasticity in an activity-dependent manner and the emergence of locomotor training it is now important to examine how group III and IV afferents respond to exercise after SCI. Dietz et al., has shown that during locomotor training patients with severe chronic SCI have muscle EMG responses even though their limbs are moved passively by a physical therapist. The EMG pattern is actually quite coordinated and resembles EMG activity during locomotion in uninjured human suggesting that the CPG is being activated [151]. However, after 10 minutes this EMG activity is significantly diminished despite the continuation of passive movements [167]. The mechanisms for this phenomenon, termed "EMG exhaustion", remain unknown but generally it is explained by neuronal dysfunction after SCI [166]. Since skeletal muscle is activated during locomotor training, consideration of how this activity may play a role in EMG exhaustion is warranted. It has been shown that skeletal muscles in the limbs below the level of an SCI not only undergo significant atrophy but the muscle fiber types shift from fatigue-resistant (type I) to easily fatigable (type II) [328]. Could EMG exhaustion be at least partly explained by the "exhausted" muscles that "communicate" their state through group III and 
IV afferents to the CNS? What outcome should we expect if even in the uninjured state the group III and IV signaling leads to a diminished output through inhibition of descending motor drive, motoneurons and other proprioceptive afferents? Taking into account that after SCI the locomotor CPG is heavily dependent on afferent input for its activation [121] and that the nociceptive afferents compose the majority of sensory innervation of the skeletal muscle [264, 265], group III and IV afferent signaling could play a significant role in mediation of EMG exhaustion phenomenon.

\section{Future directions 2: stretching and cardiovascular function}

In light of the physiological effects of group III and IV afferent on the cardiovascular (CV) system it would also be important to establish their role in CV function during exercise after SCI. Since the adaptive upregulation of the cardiopulmonary responses during exercise is dependent on group III and IV afferent feedback to the supraspinal centers, loss of the ascending pathways as a result of SCI might be a limiting factor resulting in an inadequate $\mathrm{CV}$ response to the onset of muscle activity. The failure of the $\mathrm{CV}$ response thus could contribute to the early onset of metabolic-induced fatigue through an insufficient blood supply to the muscle. Increases in the metabolite concentrations could then activate nociceptive afferents and thus reduce motor output. While the initial CV upregulation response is dependent on non-nociceptor (likely group III afferents) [206], plasticity within the nociceptive afferents, specifically capsaicinsensitive [249, 329], contributes to autonomic dysreflexia (AD), a life-threatening event characterized by extreme hypertension and bradycardia [330]. AD is most often triggered by a noxious stimulus below the level of injury most frequently as a result of bladder or bowel distension [330]. Given that noxious afferents are likely activated during a muscle fatiguing exercise, it is important to establish whether SCI patients are at higher risk of 
experiencing an episode of $\mathrm{AD}$ during locomotor training. Furthermore, we have determined that stretching activates the nociceptive afferents and thus another question to investigate in the future is what effect stretching has on CV function and whether it can induce $\mathrm{AD}$ in rats with high severe thoracic SCIs.

\section{Rationale for stretching: methodological considerations}

Several studies have demonstrated that stretching can prevent the shortening of the muscle and even cause an increase in the number of sarcomeres in series in the immobilized rat and rabbit skeletal muscles $[68,87,331]$. To date, no investigations of these claims have been done in humans although non-invasive techniques such as high resolution ultrasound have emerged that make it more feasible to conduct this type of study in the human subjects [332]. Instead, much of the literature that seeks to inform clinical practice optimistically references the findings in the animal models as a rationale for inclusion of stretching into rehabilitation under the assumption that it will produce similar results in humans. As has been mentioned already, stretching has neither been found to resolve muscle contractures in patients with SCI or significantly improve the ROM as one would expect with an increase in the number of sarcomeres [97]. A potential explanation for the discrepancy between animal and human outcomes emerges upon closer examination of the methodology used in the animal studies that report positive effects of stretching on the muscle properties. An important observation was made by Baewer et al., who found that application of stretch to the cast-immobilized limbs of neurologically intact animals activates an escape response which results in the increased EMG activity in the stretched muscles [333]. Therefore, as authors from this group pointed out, it is not clear whether the reported increase in sarcomeres is due to stretch or the combination of stretch and muscle contraction. To address this uncertainty the authors designed a study in which animals were 
anesthetized in order to prevent the contractions during the stretching of the immobilized limb. The authors found that stretching in anesthetized rats (without muscle contractions) did not prevent the shortening adaptation (loss of sarcomeres) while the induction of the contraction by electrical stimulation of the stretched muscle significantly increased the number of sarcomeres [334]. Furthermore, stretching does not prevent the development of knee contracture in the rats with a thoracic spinal cord transection [72]. In our study immobility as a result of stretch-induced locomotor dysfunction led to the development of muscle contractures around the knee and hip joints [218]. Continuation of muscle stretching around the joints affected by the contracture did not improve the ROM. On the other hand, we had to stop stretching the chronic group of animals after 4 weeks because the increasing muscle atrophy and joint stiffness prevented the achievement of the full ROM required for our stretching protocol. As animals regained their locomotor function once stretching was stopped, the contractures resolved on their own and the normal ROM was restored. Thus, our data further supports the notion that stretching does not reverse muscle contracture in the SCI animals. However, we can infer that a combination of taskspecific loading of the patients' joints during locomotor training, and acquiring the ability to stand or move as a result of epidural or transcutaneous stimulation, will lead to the restoration of normal ROM in patients with SCI. Meanwhile, there needs to be further investigation into whether stretching paired with muscle contractions can be an effective intervention for the prevention and/or treatment of muscle contractures in humans with SCI. The few studies that have reported significant improvement in joint ROM as a result of serial casting occurred in neurological subjects who developed muscle contractures as a result of spasticity $[91,92]$ and thus stretching stimulus in those cases was applied in the 
presence of the muscle contraction. Based on these observations, muscle contractures that develop as a result of immobility may benefit from the combination of stretch and electrical stimulation. However, this type of intervention may lead to the DOMS as a result of eccentric muscle contractions. We have speculated that the activation and sensitization of nociceptive afferents as occurs in DOMS could be responsible for the disruption of locomotor function at least in our dynamic stretching study [254]. Therefore, if stretching in combination with muscle contraction is to be used for the treatment of muscle contractures, the assessment of the effects of such intervention on motor function should be done.

\section{How stretching brings about rapid increase in ROM in healthy humans and why it isn't effective after SCI}

Although the rationale for including stretching in rehabilitation of muscle contractures mainly comes from the animal studies $[68,87,88]$, there is also a wealth of evidence (as discussed in the introduction) from studies in healthy humans that relatively short periods of stretch can rapidly produce an increase in ROM. Why then has stretching not been an effective therapy thus far in treating of ROM impairments after SCI? Understanding the physiological mechanisms of how stretching brings about improvements in ROM can perhaps shed light onto the discrepancy in efficacy of this therapy in healthy vs injured human populations.

The most common physiological mechanisms that are implicated for stretchinduced increases in ROM in healthy subjects are the viscoelastic deformation of tissues, particularly at the muscle-tendon unit [335-337] and neuromuscular relaxation due to the autogenic inhibition and reciprocal inhibition (in proprioceptive neuromuscular facilitation (PNF) technique) [80, 338]. Danish clinical scientist Peter Magnusson has recently 
reviewed the evidence in support of these and a few other commonly proposed mechanisms and concluded that none of them can sufficiently account for long lasting stretch-mediated increases in ROM [339]. The author argues that viscoelastic deformation falls short as a potential mechanism because human subjects maintain increased ROM even when the viscoelastic properties of the muscles have returned to baseline (in his own experiments, Magnusson established that viscoelastic changes in response to stretch diminish after 10 minutes)[78]. The PNF method of stretching has become a popular kind of flexibility training as several studies demonstrated its superiority over static stretching in bringing about ROM increases [340-342]. The key in PNF stretching is a brief pre-stretch contraction of the antagonistic muscle followed by a homonymous muscle contraction [343]. The observation that this simple modification to the original static stretching method results in quick increases in ROM have been attributed to neuromuscular relaxation as a result of autogenic and reciprocal inhibition from the activation of Golgi tendon organs (GTO) and Ia afferents, respectively [343]. These explanations function under the assumption that muscle offers a large amount of reflex-mediated resistance during the initial application of stretch and thus modulation of the reflexes via a pre-stretch muscle contraction reduces reflex activity and allows a greater ROM to be achieved [339]. Several studies dispute these mechanisms however. First, a GTO related decrease in excitability (autogenic inhibition) is likely limited to the period of active muscle contraction, since no GTO activity is observed following the contraction [344, 345]. Second, studies in intact humans did not find significant EMG activity in the stretched muscle suggesting that reflex activity does not limit the achievable ROM [339, 346-348]. 
Due to the lack of supporting evidence for the commonly proposed mechanisms for stretch-mediated increases in ROM a different theory has emerged - the "sensory theory" which suggests that increases in ROM are the result of increased tolerance to stretch [78, 84, 348]. Interestingly, one study showed that stretching of one limb resulted in an increased ROM not only of the stretched limb but also the contralateral unstretched limb, further supporting the idea that neural adaptations occur in response to stretch [253]. The physiological mechanisms are still not understood, but both central and peripheral pain modulation has been implicated $[349,350]$. In research experiments on stretching the end ROM is assessed when a subject reports reaching the point of mild discomfort $[351,352]$ while some studies use "stretch to pain" [84] for the end ROM measurements. Therefore, it is likely nociceptive afferents are activated during stretching. Central processing of nociceptive information is complex [285], but increased tolerance to stretch could be due to the inhibition of nociceptive afferents through the endogenous pain gaiting system so that their threshold for firing is increased, allowing for greater ROM to be achieved without the sensation of discomfort or pain. Speculatively, muscle retains some capacity for further viscoelastic deformation at the first onset of "discomfort" during stretch and therefore, inhibition of nociceptive afferents after their initial activation could then allow the subject to move further into the stretch by tapping into the "reserved" viscoelastic properties of the muscle undergoing stretching. The effectiveness of stretching at increasing ROM then depends on the intact sensory system and muscles retaining their viscoelastic properties. Following a severe SCI, the sensory system is impaired and the paralyzed muscles lose their viscoelastic properties, which is part of the pathophysiology of muscle contracture. 
This could explain why stretching performed for 30 seconds to 1 minute is not effective at increasing ROM of a contracted muscle after SCI.

\section{Mechanism of stretch-induced strength loss and its relationship to spasticity}

As alluded to in the previous chapters, the fact that stretching causes an acute strength loss and reduces some aspects of motor performance is a well-recognized phenomenon in the sports performance field. The proposed mechanisms include both mechanical and neural factors. Some argue that increased compliance of the musculartendon unit leads to a decrease in force transmission [353]. Another theory suggests that stretching may disrupt the number of myosin-actin interactions necessary for optimal force production through cross-bridge formation during a muscle contraction [354]. However, these theories cannot account for the strength-loss (as well as increase in ROM) that occurs not only in the stretched, but also in the contralateral unstretched leg $[219,355]$. These observations implicate a neural-based mechanism for stretch-induced strength loss. One study examined the effects of repeated passive stretching of the triceps surae in healthy humans on force production and excitability of the H-reflex [224]. Along with a 23\% reduction in maximal voluntary torque production and a $19 \%$ reduction in the EMG amplitude there was a $46 \%$ decrease in H-reflex excitability (with a $43 \%$ decrease in $\mathrm{H} / \mathrm{M}$ ratio). In their conclusion the authors suggested that RPS reduces the sensitivity of the muscle spindle. Since motoneuronal excitability at least in part depends on Ia afferent feedback the resulting decrease in muscle spindle discharges then leads to the decrease in motoneuronal output. The authors argue that the decrease in muscle spindle sensitivity is due to an increased compliance of the muscle (including the intrafusal fibers which are the end organs of the Ia afferents). Another possible explanation is that stretching resulted in the activation of nociceptive afferents which have been shown to reduce the excitability of 
the monosynaptic reflex through pre-synaptic inhibition of Ia afferent terminals within the spinal cord [326, 356]. Stretching has been also reported to decrease H-reflex [357] or other reflex-based measures of motoneuronal excitability $[110,358]$ in patients with spasticity, however, others found no effect on the excitability of H-reflex [359]. These discrepancies are likely due to the heterogeneity in the study designs particularly as it relates to the dosage of stretching as well as the inherent variability due to the type of injury and spasticity severity in the subject population. Spasticity is a multidimensional disorder which reflects the complexity of the neural circuitry and its capacity for compensatory plasticity following the CNS damage. Stretching thus may reduce spasticity by inhibiting the signaling of one of the prominent excitatory modulators of motoneuronal activity - muscle spindle. However, whether stretching can reverse the molecular changes that take place at the motoneuronal level such as an upregulation of constitutively active 5-HT receptors and downregulation of $\mathrm{KCC} 2$ channels is not clear. Understanding the mechanisms by which stretching can potentially reduce spasticity and whether there are any long term benefits or detriments of such approach needs to be further investigated.

\section{Future of stretching therapy in rehabilitation after SCI}

Muscle contractures and spasticity are two major and common complications following SCI and other neurologic damage that pose a significant disruption to patient's ADL and overall quality of life. Stretching traditionally has been the number one therapy to treat/prevent both muscle contractures and spasticity [73]. At first glance, the rationale for including stretching in the management of muscle contractures after SCI appears sound; however, several large literature reviews found no evidence for the effectiveness of stretching [97, 98]. The failure to observe similar effects of stretching on muscle properties as reported by Williams et al., in rats likely results from an inadvertent omission of subtle 
observations such as muscle contractions occurring simultaneously with the stretching applied to the cast-immobilized limb. As it became apparent in later more carefully designed studies, muscle contraction during stretching is the key to the increased number of sarcomeres, as passive stretching alone does not prevent shortening of the immobilized muscle [334]. Nevertheless, stretching has become a leading therapy for the treatment of contractures as a result of overly enthusiastic extrapolation of the misleading results to human subjects without proper clinical trials to confirm the effectiveness of stretching. What about the observation that stretching is effective at increasing ROM in healthy humans? Most studies do not use this as a rationale for the treatment of contractures and rightfully so, as it may not be translatable to the people with SCI. Based on the "sensory theory" that seeks to explain the mechanism underlying a stretch-induced increase in ROM [339] in the intact human, stretching effectiveness for increasing the ROM likely depends on intact sensory perception, the descending pain modulatory system as well as viscoelastic properties of the muscle. Severe SCI eventually results in the loss of all of these components which potentially explains the ineffectiveness of stretching at combating the pathophysiological changes that lead to muscle contracture. On the other hand, stretching might be helpful at temporarily reducing spasticity [110], presumably by reducing the excitatory input from proprioceptive afferents [224]. Stretching also appears to be more effective at improving spasticity-induced muscle contractures but any long term effects of the combination of stretching and muscle contraction on motor function need to be elucidated. The data from the stretching projects in the Magnuson laboratory brings in a concerning possibility that stretching might have a negative effect on locomotor/motor function and recovery post-SCI. The extent of the clinical relevance of this phenomenon 
still needs to be established, however, considering all the evidence presented throughout the thesis a few conclusions and suggestions can be made.

First, it is important to re-emphasize that muscles and joints receive a lot of sensory innervation which is predominantly composed of nociceptive afferents (at least based on the composition of the nerves from animal models). Thus, when manipulating a subject's limbs while treating a contracture or a spasm, physical therapists should consider which type of sensory afferents will be activated and what type of feedback they take back to the spinal cord. This can be challenging since a lot of patients lack sensory function below the level of injury and thus can give no verbal feedback. Harvey et al., has demonstrated that some PTs apply torques that would be intolerable to intact individuals [211]. Based on the evidence from basic science studies it is clear that nociceptive afferent signaling can disrupt aspects of adaptive plasticity, such as spinal learning, and we specifically demonstrate that stretch-induced locomotor deficits are dependent on activation of nociceptive afferents. Thus, the question is raised - should stretching therapy as currently practiced be continued? From our perspective the answer is no for two simple reasons. First, the lack of evidence for its effectiveness and, second, that it may result in the activation of nociceptive afferent signaling, which has been shown to induce inflammation in the spinal cord [301, 360]. With that said, we would not discourage further investigation into the effects of stretching with concomitant muscle contraction on muscle contracture, as it may have potential benefits for the management of this very real, and in some cases debilitating issue. The concern for activation of nociceptive afferents with this treatment still remains; however, as we conduct our next stretching experiments we hope to find a pharmacological agent that can mitigate the negative effects of nociceptive afferents in the spinal cord. For 
example, if our hypothesis of involvement of endogenous opioids in mediation of stretchinduced deficits is confirmed, then administration of naloxone or naltrexone before, during or after stretching + muscle contraction protocol for the treatment of muscle contractures can also prevent detrimental effects associated with nociceptive activation in humans.

Second, whereas in the past spinal cord injury meant paralysis, as a field we now clearly realize that the spinal cord, even in the absence of the majority of supraspinal input, is capable of substantial plasticity and function. If we can successfully determine a safe approach to modulate the excitability of the spinal cord below the level of lesion with the advancement of technology we will likely restore some locomotor function. In this case muscle contractures may resolve as a result of active load bearing, and stretching may no longer be needed.

Finally, rehabilitation after SCI has always been done with the goal of helping patients. It would be understandably challenging to admit that we might have employed a counterproductive measure if in the future stretching is found to be detrimental to the functional recovery of patients with SCI. However, it is imperative that we keep an open mind, learn from mistakes and continued research, and seek to refine our understanding in order to bring the most current evidence-based practice to patients. 


\section{REFERENCES}

1. National Spinal Cord Injury Statistical Center, B., AL; University of Alabama at Birmingham, Facts and Figures at a Glance. 2016.

2. Illis, L.S., Central nervous system regeneration does not occur. Spinal Cord, 2012. 50(4): p. 259-63.

3. Hodgetts, S.I. and A.R. Harvey, Neurotrophic Factors Used to Treat Spinal Cord Injury. Vitam Horm, 2017. 104: p. 405-457.

4. Lacroix, S. and M.H. Tuszynski, Neurotrophic factors and gene therapy in spinal cord injury. Neurorehabil Neural Repair, 2000. 14(4): p. 265-75.

5. Pearse, D.D., et al., Transplantation of Schwann cells and/or olfactory ensheathing glia into the contused spinal cord: Survival, migration, axon association, and functional recovery. Glia, 2007. 55(9): p. 976-1000.

6. Barakat, D.J., et al., Survival, integration, and axon growth support of glia transplanted into the chronically contused spinal cord. Cell Transplant, 2005. 14(4): p. 225-40.

7. Lee, S.H., et al., Impact of local injection of brain-derived neurotrophic factorexpressing mesenchymal stromal cells (MSCs) combined with intravenous MSC delivery in a canine model of chronic spinal cord injury. Cytotherapy, 2016.

8. Abbaszadeh, H.A., et al., Human ciliary neurotrophic factor-overexpressing stable bone marrow stromal cells in the treatment of a rat model of traumatic spinal cord injury. Cytotherapy, 2015. 17(7): p. 912-21. 
9. Schwab, M.E., Repairing the injured spinal cord. Science, 2002. 295(5557): p. 1029-31.

10. Liu, K., et al., PTEN deletion enhances the regenerative ability of adult corticospinal neurons. Nat Neurosci, 2010. 13(9): p. 1075-81.

11. Kadoya, K., et al., Combined intrinsic and extrinsic neuronal mechanisms facilitate bridging axonal regeneration one year after spinal cord injury. Neuron, 2009. 64(2): p. 165-72.

12. Tsai, E.C., A.V. Krassioukov, and C.H. Tator, Corticospinal regeneration into lumbar grey matter correlates with locomotor recovery after complete spinal cord transection and repair with peripheral nerve grafts, fibroblast growth factor 1, fibrin glue, and spinal fusion. J Neuropathol Exp Neurol, 2005. 64(3): p. 230-44.

13. Badner, A., A.M. Siddiqui, and M.G. Fehlings, Spinal Cord Injuries: How Could Cell Therapy Help? Expert Opin Biol Ther, 2017.

14. Wolman, L., THE DISTURBANCE OF CIRCULATION IN TRAUMATIC PARAPLEGIA IN ACUTE AND LATE STAGES: A PATHOLOGICAL STUDY. Paraplegia, 1965. 2: p. 213-26.

15. Dumont, R.J., et al., Acute spinal cord injury, part I: pathophysiologic mechanisms. Clin Neuropharmacol, 2001. 24(5): p. 254-64.

16. Kwon, B.K., et al., Pathophysiology and pharmacologic treatment of acute spinal cord injury. Spine J, 2004. 4(4): p. 451-64.

17. Sandler, A.N. and C.H. Tator, Review of the effect of spinal cord trama on the vessels and blood flow in the spinal cord. J Neurosurg, 1976. 45(6): p. 638-46. 
18. Tator, C.H. and M.G. Fehlings, Review of the secondary injury theory of acute spinal cord trauma with emphasis on vascular mechanisms. J Neurosurg, 1991. 75(1): p. 15-26.

19. Smith, S.L., et al., Direct measurement of hydroxyl radicals, lipid peroxidation, and blood-brain barrier disruption following unilateral cortical impact head injury in the rat. J Neurotrauma, 1994. 11(4): p. 393-404.

20. Faden, A.I., et al., N-methyl-D-aspartate antagonist MK801 improves outcome following traumatic spinal cord injury in rats: behavioral, anatomic, and neurochemical studies. J Neurotrauma, 1988. 5(1): p. 33-45.

21. Choi, D.W., Excitotoxic cell death. J Neurobiol, 1992. 23(9): p. 1261-76.

22. Mody, I. and J.F. MacDonald, NMDA receptor-dependent excitotoxicity: the role of intracellular Ca2+ release. Trends Pharmacol Sci, 1995. 16(10): p. 356-9.

23. Wrathall, J.R., Y.D. Teng, and D. Choiniere, Amelioration of functional deficits from spinal cord trauma with systemically administered NBQX, an antagonist of non-N-methyl-D-aspartate receptors. Exp Neurol, 1996. 137(1): p. 119-26.

24. Shields, D.C., et al., Calpain activity and expression increased in activated glial and inflammatory cells in penumbra of spinal cord injury lesion. J Neurosci Res, 2000. 61(2): p. 146-50.

25. Hall, E.D. and D.L. Wolf, A pharmacological analysis of the pathophysiological mechanisms of posttraumatic spinal cord ischemia. J Neurosurg, 1986. 64(6): p. 951-61.

26. Fiskum, G., Mitochondrial participation in ischemic and traumatic neural cell death. J Neurotrauma, 2000. 17(10): p. 843-55. 
27. Janeway, C.A., Jr., The immune system evolved to discriminate infectious nonself from noninfectious self. Immunol Today, 1992. 13(1): p. 11-6.

28. Eigenbrod, T., et al., Cutting edge: critical role for mesothelial cells in necrosisinduced inflammation through the recognition of IL-1 alpha released from dying cells. J Immunol, 2008. 181(12): p. 8194-8.

29. Schmitz, J., et al., IL-33, an interleukin-1-like cytokine that signals via the IL-1 receptor-related protein ST2 and induces Thelper type 2-associated cytokines. Immunity, 2005. 23(5): p. 479-90.

30. Kim, S.M., et al., Hyperuricemia-induced NLRP3 activation of macrophages contributes to the progression of diabetic nephropathy. Am J Physiol Renal Physiol, 2015. 308(9): p. F993-f1003.

31. Yawata, I., et al., Macrophage-induced neurotoxicity is mediated by glutamate and attenuated by glutaminase inhibitors and gap junction inhibitors. Life Sci, 2008. 82(21-22): p. 1111-6.

32. Shechter, R., et al., Infiltrating blood-derived macrophages are vital cells playing an anti-inflammatory role in recovery from spinal cord injury in mice. PLoS Med, 2009. 6(7): p. e1000113.

33. Di Virgilio, F., Liaisons dangereuses: P2X(7) and the inflammasome. Trends Pharmacol Sci, 2007. 28(9): p. 465-72.

34. Lukens, J.R., J.M. Gross, and T.D. Kanneganti, IL-1 family cytokines trigger sterile inflammatory disease. Front Immunol, 2012. 3: p. 315.

35. Roth, T.L., et al., Transcranial amelioration of inflammation and cell death after brain injury. Nature, 2014. 505(7482): p. 223-8. 
36. Tonai, T., et al., A neutrophil elastase inhibitor (ONO-5046) reduces neurologic damage after spinal cord injury in rats. J Neurochem, 2001. 78(5): p. 1064-72.

37. Gorio, A., et al., Reparixin, an inhibitor of CXCR2 function, attenuates inflammatory responses and promotes recovery of function after traumatic lesion to the spinal cord. J Pharmacol Exp Ther, 2007. 322(3): p. 973-81.

38. Kroner, A., et al., TNF and increased intracellular iron alter macrophage polarization to a detrimental M1 phenotype in the injured spinal cord. Neuron, 2014. 83(5): p. 1098-116.

39. Dougherty, K.D., C.F. Dreyfus, and I.B. Black, Brain-derived neurotrophic factor in astrocytes, oligodendrocytes, and microglia/macrophages after spinal cord injury. Neurobiol Dis, 2000. 7(6 Pt B): p. 574-85.

40. Yin, Y., et al., Oncomodulin is a macrophage-derived signal for axon regeneration in retinal ganglion cells. Nat Neurosci, 2006. 9(6): p. 843-52.

41. Bomstein, Y., et al., Features of skin-coincubated macrophages that promote recovery from spinal cord injury. J Neuroimmunol, 2003. 142(1-2): p. 10-6.

42. Schwartz, M., "Tissue-repairing" blood-derived macrophages are essential for healing of the injured spinal cord: from skin-activated macrophages to infiltrating blood-derived cells? Brain Behav Immun, 2010. 24(7): p. 1054-7.

43. Gadani, S.P., et al., Dealing with Danger in the CNS: The Response of the Immune System to Injury. Neuron, 2015. 87(1): p. 47-62.

44. Dietz, V., Neuronal plasticity after a human spinal cord injury: positive and negative effects. Exp Neurol, 2012. 235(1): p. 110-5. 
45. Yarkony, G.M., et al., Contractures complicating spinal cord injury: incidence and comparison between spinal cord centre and general hospital acute care. Paraplegia, 1985. 23(5): p. 265-71.

46. Maynard, F.M., R.S. Karunas, and W.P. Waring, 3rd, Epidemiology of spasticity following traumatic spinal cord injury. Arch Phys Med Rehabil, 1990. 71(8): p. 566-9.

47. Grover, J., H. Gellman, and R.L. Waters, The effect of a flexion contracture of the elbow on the ability to transfer in patients who have quadriplegia at the sixth cervical level. J Bone Joint Surg Am, 1996. 78(9): p. 1397-400.

48. Cooper, J.E., et al., Elbow joint restriction: effect on functional upper limb motion during performance of three feeding activities. Arch Phys Med Rehabil, 1993. 74(8): p. 805-9.

49. Harvey, L. and J. Crosbie, Effect of elbow flexion contractures on the ability of people with C5 and C6 tetraplegia to lift. Physiother Res Int, 2001. 6(2): p. 76-82.

50. Welmer, A.K., et al., Spasticity and its association with functioning and healthrelated quality of life 18 months after stroke. Cerebrovasc Dis, 2006. 21(4): p. 247-53.

51. Katz, R.T. and W.Z. Rymer, Spastic hypertonia: mechanisms and measurement. Arch Phys Med Rehabil, 1989. 70(2): p. 144-55.

52. Morita, H., et al., Modulation of presynaptic inhibition and disynaptic reciprocal Ia inhibition during voluntary movement in spasticity. Brain, 2001. 124(Pt 4): p. 826-37. 
53. Nielsen, J., N. Petersen, and C. Crone, Changes in transmission across synapses of Ia afferents in spastic patients. Brain, 1995. 118 ( Pt 4): p. 995-1004.

54. Roy, R.R. and V.R. Edgerton, Neurobiological perspective of spasticity as occurs after a spinal cord injury. Exp Neurol, 2012. 235(1): p. 116-22.

55. Calancie, B., Spinal myoclonus after spinal cord injury. J Spinal Cord Med, 2006. 29(4): p. 413-24.

56. Heckmann, C.J., M.A. Gorassini, and D.J. Bennett, Persistent inward currents in motoneuron dendrites: implications for motor output. Muscle Nerve, 2005. 31(2): p. $135-56$.

57. ElBasiouny, S.M., J.E. Schuster, and C.J. Heckman, Persistent inward currents in spinal motoneurons: important for normal function but potentially harmful after spinal cord injury and in amyotrophic lateral sclerosis. Clin Neurophysiol, 2010. 121(10): p. 1669-79.

58. Harvey, P.J., et al., 5-HT2 receptor activation facilitates a persistent sodium current and repetitive firing in spinal motoneurons of rats with and without chronic spinal cord injury. J Neurophysiol, 2006. 96(3): p. 1158-70.

59. Lee, R.H. and C.J. Heckman, Enhancement of bistability in spinal motoneurons in vivo by the noradrenergic alphal agonist methoxamine. J Neurophysiol, 1999. 81(5): p. 2164-74.

60. Ditunno, J.F., et al., Spinal shock revisited: a four-phase model. Spinal Cord, 2004. 42(7): p. 383-95.

61. Murray, K.C., et al., Motoneuron excitability and muscle spasms are regulated by 5-HT2B and 5-HT2C receptor activity. J Neurophysiol, 2011. 105(2): p. 731-48. 
62. Curtis, D.R., Pharmacological investigations upon inhibition of spinal motoneurones. J Physiol, 1959. 145(1): p. 175-92.

63. Boulenguez, P., et al., Down-regulation of the potassium-chloride cotransporter KCC2 contributes to spasticity after spinal cord injury. Nat Med, 2010. 16(3): p. 302-7.

64. Appell, H.J., Muscular atrophy following immobilisation. A review. Sports Med, 1990. 10(1): p. 42-58.

65. Dudley-Javoroski, S. and R.K. Shields, Muscle and bone plasticity after spinal cord injury: review of adaptations to disuse and to electrical muscle stimulation. $\mathrm{J}$ Rehabil Res Dev, 2008. 45(2): p. 283-96.

66. Dalyan, M., A. Sherman, and D.D. Cardenas, Factors associated with contractures in acute spinal cord injury. Spinal Cord, 1998. 36(6): p. 405-8.

67. Biolo, G., et al., Metabolic consequences of physical inactivity. J Ren Nutr, 2005. 15(1): p. 49-53.

68. Williams, P.E., Use of intermittent stretch in the prevention of serial sarcomere loss in immobilised muscle. Ann Rheum Dis, 1990. 49(5): p. 316-7.

69. Smith, L.R., et al., Hamstring contractures in children with spastic cerebral palsy result from a stiffer extracellular matrix and increased in vivo sarcomere length. $\mathrm{J}$ Physiol, 2011. 589(Pt 10): p. 2625-39.

70. Sakamoto, J., et al., Immobilization-induced cartilage degeneration mediated through expression of hypoxia-inducible factor-1alpha, vascular endothelial growth factor, and chondromodulin-I. Connect Tissue Res, 2009. 50(1): p. 37-45. 
71. Moriyama, H., et al., Alteration of knee joint connective tissues during contracture formation in spastic rats after an experimentally induced spinal cord injury. Connect Tissue Res, 2007. 48(4): p. 180-7.

72. Moriyama, H., et al., Amount of torque and duration of stretching affects correction of knee contracture in a rat model of spinal cord injury. Clin Orthop Relat Res, 2013. 471(11): p. 3626-36.

73. Harvey, L.A. and R.D. Herbert, Muscle stretching for treatment and prevention of contracture in people with spinal cord injury. Spinal Cord, 2002. 40(1): p. 1-9.

74. Huddleston, O.L., FLEXIBILITY EXERCISES FOR PHYSICAL FITNESS. Arch Phys Med Rehabil, 1964. 45: p. 581-4.

75. Anderson, B. and E.R. Burke, Scientific, medical, and practical aspects of stretching. Clin Sports Med, 1991. 10(1): p. 63-86.

76. Davis, D.S., et al., The effectiveness of 3 stretching techniques on hamstring flexibility using consistent stretching parameters. J Strength Cond Res, 2005. 19(1): p. 27-32.

77. Page, P., Current concepts in muscle stretching for exercise and rehabilitation. Int J Sports Phys Ther, 2012. 7(1): p. 109-19.

78. Magnusson, S.P., et al., A biomechanical evaluation of cyclic and static stretch in human skeletal muscle. Int J Sports Med, 1998. 19(5): p. 310-6.

79. Nishikawa, Y., et al., Immediate effect of passive and active stretching on hamstrings flexibility: a single-blinded randomized control trial. J Phys Ther Sci, 2015. 27(10): p. 3167-70. 
80. Tanigawa, M.C., Comparison of the hold-relax procedure and passive mobilization on increasing muscle length. Phys Ther, 1972. 52(7): p. 725-35.

81. Bandy, W.D. and J.M. Irion, The effect of time on static stretch on the flexibility of the hamstring muscles. Phys Ther, 1994. 74(9): p. 845-50; discussion 850-2.

82. Bandy, W.D., J.M. Irion, and M. Briggler, The effect of time and frequency of static stretching on flexibility of the hamstring muscles. Phys Ther, 1997. 77(10): p. 1090-6.

83. Bandy, W.D., J.M. Irion, and M. Briggler, The effect of static stretch and dynamic range of motion training on the flexibility of the hamstring muscles. J Orthop Sports Phys Ther, 1998. 27(4): p. 295-300.

84. Halbertsma, J.P. and L.N. Goeken, Stretching exercises: effect on passive extensibility and stiffness in short hamstrings of healthy subjects. Arch Phys Med Rehabil, 1994. 75(9): p. 976-81.

85. Cini, A., G.S. de Vasconcelos, and C.S. Lima, Acute effect of different time periods of passive static stretching on the hamstring flexibility. J Back Musculoskelet Rehabil, 2016.

86. Medeiros, D.M., et al., Influence of static stretching on hamstring flexibility in healthy young adults: Systematic review and meta-analysis. Physiother Theory Pract, 2016. 32(6): p. 438-445.

87. Williams, P.E., Effect of intermittent stretch on immobilised muscle. Ann Rheum Dis, 1988. 47(12): p. 1014-6. 
88. Williams, P.E., et al., The importance of stretch and contractile activity in the prevention of connective tissue accumulation in muscle. J Anat, 1988. 158: p. 109-14.

89. Tardieu, C., et al., Adaptation of connective tissue length to immobilization in the lengthened and shortened positions in cat soleus muscle. J Physiol (Paris), 1982. 78(2): p. 214-20.

90. Goldspink, D.F., The influence of immobilization and stretch on protein turnover of rat skeletal muscle. J Physiol, 1977. 264(1): p. 267-82.

91. Booth, B.J., M. Doyle, and J. Montgomery, Serial casting for the management of spasticity in the head-injured adult. Phys Ther, 1983. 63(12): p. 1960-6.

92. Anderson, J.P., et al., Efficacy of soft splints in reducing severe knee-flexion contractures. Dev Med Child Neurol, 1988. 30(4): p. 502-8.

93. Gelinas, J.J., et al., The effectiveness of turnbuckle splinting for elbow contractures. J Bone Joint Surg Br, 2000. 82(1): p. 74-8.

94. Harvey, L.A., et al., A randomized trial assessing the effects of 4 weeks of daily stretching on ankle mobility in patients with spinal cord injuries. Arch Phys Med Rehabil, 2000. 81(10): p. 1340-7.

95. Harvey, L.A., et al., Randomised trial of the effects offour weeks of daily stretch on extensibility of hamstring muscles in people with spinal cord injuries. Aust $\mathrm{J}$ Physiother, 2003. 49(3): p. 176-81.

96. Harvey, L., et al., Twelve weeks of nightly stretch does not reduce thumb webspace contractures in people with a neurological condition: a randomised controlled trial. Aust J Physiother, 2006. 52(4): p. 251-8. 
97. Katalinic, O.M., L.A. Harvey, and R.D. Herbert, Effectiveness of stretch for the treatment and prevention of contractures in people with neurological conditions: a systematic review. Phys Ther, 2011.91(1): p. 11-24.

98. Katalinic, O.M., et al., Stretch for the treatment and prevention of contractures. Cochrane Database Syst Rev, 2010(9): p. Cd007455.

99. Bovend'Eerdt, T.J., et al., The effects of stretching in spasticity: a systematic review. Arch Phys Med Rehabil, 2008. 89(7): p. 1395-406.

100. Odeen, I. and E. Knutsson, Evaluation of the effects of muscle stretch and weight load in patients with spastic paraplegia. Scand J Rehabil Med, 1981. 13(4): p. 117-21.

101. Odeen, I., Reduction of muscular hypertonus by long-term muscle stretch. Scand J Rehabil Med, 1981. 13(2-3): p. 93-9.

102. Ashworth, B., PRELIMINARY TRIAL OF CARISOPRODOL IN MULTIPLE SCLEROSIS. Practitioner, 1964. 192: p. 540-2.

103. Bohannon, R.W. and M.B. Smith, Interrater reliability of a modified Ashworth scale of muscle spasticity. Phys Ther, 1987. 67(2): p. 206-7.

104. Fugl-Meyer, A.R., et al., The post-stroke hemiplegic patient. 1. a method for evaluation of physical performance. Scand J Rehabil Med, 1975. 7(1): p. 13-31.

105. Jo, H.M., J.C. Song, and S.H. Jang, Improvements in spasticity and motor function using a static stretching device for people with chronic hemiparesis following stroke. NeuroRehabilitation, 2013. 32(2): p. 369-75. 
106. de Jong, L.D., A. Nieuwboer, and G. Aufdemkampe, Contracture preventive positioning of the hemiplegic arm in subacute stroke patients: a pilot randomized controlled trial. Clin Rehabil, 2006. 20(8): p. 656-67.

107. Bressel, E. and P.J. McNair, The effect of prolonged static and cyclic stretching on ankle joint stiffness, torque relaxation, and gait in people with stroke. Phys Ther, 2002. 82(9): p. 880-7.

108. Waldman, G., et al., Effects of robot-guided passive stretching and active movement training of ankle and mobility impairments in stroke.

NeuroRehabilitation, 2013. 32(3): p. 625-34.

109. Zhou, Z., et al., Robot-Assisted Rehabilitation of Ankle Plantar Flexors Spasticity: A 3-Month Study with Proprioceptive Neuromuscular Facilitation. Front Neurorobot, 2016. 10: p. 16.

110. Estes, S.P., J.A. Iddings, and E.C. Field-Fote, Priming Neural Circuits to Modulate Spinal Reflex Excitability. Front Neurol, 2017. 8: p. 17.

111. Drew, T. and D.S. Marigold, Taking the next step: cortical contributions to the control of locomotion. Curr Opin Neurobiol, 2015. 33: p. 25-33.

112. Garcia-Rill, E., The basal ganglia and the locomotor regions. Brain Res, 1986. 396(1): p. 47-63.

113. Grillner, S. and B. Robertson, The basal ganglia downstream control of brainstem motor centres--an evolutionarily conserved strategy. Curr Opin Neurobiol, 2015. 33: p. 47-52.

114. Jordan, L.M., et al., Descending command systems for the initiation of locomotion in mammals. Brain Res Rev, 2008. 57(1): p. 183-91. 
115. Sherman, D., et al., Anatomical Location of the Mesencephalic Locomotor Region and Its Possible Role in Locomotion, Posture, Cataplexy, and Parkinsonism. Front Neurol, 2015. 6: p. 140.

116. Markin, S.N., et al., Motoneuronal and muscle synergies involved in cat hindlimb control during fictive and real locomotion: a comparison study. J Neurophysiol, 2012. 107(8): p. 2057-71.

117. Wenger, N., et al., Spatiotemporal neuromodulation therapies engaging muscle synergies improve motor control after spinal cord injury. Nat Med, 2016. 22(2): p. $138-45$.

118. Grillner, S., The motor infrastructure: from ion channels to neuronal networks. Nat Rev Neurosci, 2003. 4(7): p. 573-86.

119. Kiehn, O., Locomotor circuits in the mammalian spinal cord. Annu Rev Neurosci, 2006. 29: p. 279-306.

120. Pearson, K.G., Generating the walking gait: role of sensory feedback. Prog Brain Res, 2004. 143: p. 123-9.

121. Rossignol, S. and A. Frigon, Recovery of locomotion after spinal cord injury: some facts and mechanisms. Annu Rev Neurosci, 2011. 34: p. 413-40.

122. Sherrington, C.S., Flexion-reflex of the limb, crossed extension-reflex, and reflex stepping and standing. J Physiol, 1910. 40(1-2): p. 28-121.

123. Brown, G.T., The Intrinsic Factors in the Act of Progression in the Mammal. Proceedings of the Royal Society of London, 1911. 84(574): p. 308-319.

124. Mulloney, B. and C. Smarandache, Fifty Years of CPGs: Two Neuroethological Papers that Shaped the Course of Neuroscience. Front Behav Neurosci, 2010. 4. 
125. Wiersma, G.M.H.a.C.A., The coordination of swimmeret movements in the crayfish, Procambarus Clark II (Girard). Experimental Biology, 1960. 37(4).

126. Wilson, D.M., The Central Nervous Control of Flight in a Locust. Experimental Biology, 1961. 38: p. 471-490.

127. Kristan, W.B., Jr. and R.L. Calabrese, Rhythmic swimming activity in neurones of the isolated nerve cord of the leech. J Exp Biol, 1976. 65(3): p. 643-68.

128. Pearson, K.G., Central programming and reflex control of walking in the cockroach. Experimental Biology, 1972. 56: p. 173-193.

129. Cohen, A.H. and P. Wallen, The neuronal correlate of locomotion in fish. "Fictive swimming" induced in an in vitro preparation of the lamprey spinal cord. Exp Brain Res, 1980. 41(1): p. 11-8.

130. Pearson, K.G. and S. Rossignol, Fictive motor patterns in chronic spinal cats. J Neurophysiol, 1991. 66(6): p. 1874-87.

131. Jankowska, E., et al., The effect of DOPA on the spinal cord. 5. Reciprocal organization of pathways transmitting excitatory action to alpha motoneurones of flexors and extensors. Acta Physiol Scand, 1967. 70(3): p. 369-88.

132. Jankowska, E., et al., The effect of DOPA on the spinal cord. 6. Half-centre organization of interneurones transmitting effects from the flexor reflex afferents. Acta Physiol Scand, 1967. 70(3): p. 389-402.

133. Yang, J.F., et al., Infant stepping: a window to the behaviour of the human pattern generator for walking. Can J Physiol Pharmacol, 2004. 82(8-9): p. 662-74.

134. Dietz, V., Spinal cord pattern generators for locomotion. Clin Neurophysiol, 2003. 114(8): p. 1379-89. 
135. Forssberg, H., et al., The locomotion of the low spinal cat. II. Interlimb coordination. Acta Physiol Scand, 1980. 108(3): p. 283-95.

136. Miller, S. and F.G. van der Meche, Coordinated stepping of all four limbs in the high spinal cat. Brain Res, 1976. 109(2): p. 395-8.

137. McCrea, D.A. and I.A. Rybak, Organization of mammalian locomotor rhythm and pattern generation. Brain Res Rev, 2008. 57(1): p. 134-46.

138. McCrea, D.A. and I.A. Rybak, Modeling the mammalian locomotor CPG: insights from mistakes and perturbations. Prog Brain Res, 2007. 165: p. 235-53.

139. Rybak, I.A., K.J. Dougherty, and N.A. Shevtsova, Organization of the Mammalian Locomotor CPG: Review of Computational Model and Circuit Architectures Based on Genetically Identified Spinal Interneurons(1,2,3). eNeuro, 2015. 2(5).

140. Guertin, P.A., Preclinical evidence supporting the clinical development of central pattern generator-modulating therapies for chronic spinal cord-injured patients. Front Hum Neurosci, 2014. 8: p. 272.

141. Forssberg, H., Ontogeny of human locomotor control. I. Infant stepping, supported locomotion and transition to independent locomotion. Exp Brain Res, 1985. 57(3): p. 480-93.

142. Yakovlev PL, L.A., The myelogenetic cycles of regional maturation of the brain. Resional development of the brain in early life, 1967: p. 3-70.

143. Calancie, B., et al., Involuntary stepping after chronic spinal cord injury. Evidence for a central rhythm generator for locomotion in man. Brain, 1994. 117 ( Pt 5): p. 1143-59. 
144. Dimitrijevic, M.R., Y. Gerasimenko, and M.M. Pinter, Evidence for a spinal central pattern generator in humans. Ann N Y Acad Sci, 1998. 860: p. 360-76.

145. Gurfinkel, V.S., et al., Locomotor-like movements evoked by leg muscle vibration in humans. Eur J Neurosci, 1998. 10(5): p. 1608-12.

146. Selionov, V.A., et al., Tonic central and sensory stimuli facilitate involuntary airstepping in humans. J Neurophysiol, 2009. 101(6): p. 2847-58.

147. Gerasimenko, Y., et al., Novel and direct access to the human locomotor spinal circuitry. J Neurosci, 2010. 30(10): p. 3700-8.

148. Belanger, M., et al., A comparison of treadmill locomotion in adult cats before and after spinal transection. J Neurophysiol, 1996. 76(1): p. 471-91.

149. De Leon, R.D., et al., Retention of hindlimb stepping ability in adult spinal cats after the cessation of step training. J Neurophysiol, 1999. 81(1): p. 85-94.

150. de Leon, R.D., et al., Hindlimb locomotor and postural training modulates glycinergic inhibition in the spinal cord of the adult spinal cat. J Neurophysiol, 1999. 82(1): p. 359-69.

151. Dietz, V., et al., Locomotor capacity of spinal cord in paraplegic patients. Ann Neurol, 1995. 37(5): p. 574-82.

152. Barbeau, H. and J. Fung, The role of rehabilitation in the recovery of walking in the neurological population. Curr Opin Neurol, 2001. 14(6): p. 735-40.

153. Behrman, A.L. and S.J. Harkema, Locomotor training after human spinal cord injury: a series of case studies. Phys Ther, 2000. 80(7): p. 688-700.

154. Barbeau, H., M. Wainberg, and L. Finch, Description and application of a system for locomotor rehabilitation. Med Biol Eng Comput, 1987. 25(3): p. 341-4. 
155. Alcobendas-Maestro, M., et al., Lokomat robotic-assisted versus overground training within 3 to 6 months of incomplete spinal cord lesion: randomized controlled trial. Neurorehabil Neural Repair, 2012. 26(9): p. 1058-63.

156. Mehrholz, J., J. Kugler, and M. Pohl, Locomotor training for walking after spinal cord injury. Cochrane Database Syst Rev, 2012. 11: p. Cd006676.

157. Trimble, M.H., et al., Acute effects of locomotor training on overground walking speed and H-reflex modulation in individuals with incomplete spinal cord injury. J Spinal Cord Med, 2001. 24(2): p. 74-80.

158. Mastos, M., et al., Goal-directed training: linking theories of treatment to clinical practice for improved functional activities in daily life. Clin Rehabil, 2007. 21(1): p. 47-55.

159. Field-Fote, E.C. and K.E. Roach, Influence of a locomotor training approach on walking speed and distance in people with chronic spinal cord injury: a randomized clinical trial. Phys Ther, 2011.91(1): p. 48-60.

160. Forrest, G.F., et al., Neuromotor and musculoskeletal responses to locomotor training for an individual with chronic motor complete AIS-B spinal cord injury. $\mathrm{J}$ Spinal Cord Med, 2008. 31(5): p. 509-21.

161. Dobkin, B.H., et al., Modulation of locomotor-like EMG activity in subjects with complete and incomplete spinal cord injury. J Neurol Rehabil, 1995. 9(4): p. 18390.

162. Cote, M.P., M. Murray, and M.A. Lemay, Rehabilitation Strategies after Spinal Cord Injury: Inquiry into the Mechanisms of Success and Failure. J Neurotrauma, 2016. 
163. Wirz, M., G. Colombo, and V. Dietz, Long term effects of locomotor training in spinal humans. J Neurol Neurosurg Psychiatry, 2001. 71(1): p. 93-6.

164. de Leon, R. and C. Dy, What did we learn from the animal studies of body weight supported treadmill training and where do we go from here? J Neurotrauma, 2016.

165. Caudle, K.L., et al., Hindlimb immobilization in a wheelchair alters functional recovery following contusive spinal cord injury in the adult rat. Neurorehabil Neural Repair, 2011. 25(8): p. 729-39.

166. Dietz, V., Behavior of spinal neurons deprived of supraspinal input. Nat Rev Neurol, 2010. 6(3): p. 167-74.

167. Dietz, V. and R. Muller, Degradation of neuronal function following a spinal cord injury: mechanisms and countermeasures. Brain, 2004. 127(Pt 10): p. 222131.

168. Angeli, C.A., et al., Altering spinal cord excitability enables voluntary movements after chronic complete paralysis in humans. Brain, 2014. 137(Pt 5): p. 1394-409.

169. Gorodnichev, R.M., et al., [Transcutaneous electrical stimulation of the spinal cord: non-invasive tool for activation of locomotor circuitry in human]. Fiziol Cheloveka, 2012. 38(2): p. 46-56.

170. Gerasimenko, Y.P., et al., Noninvasive Reactivation of Motor Descending Control after Paralysis. J Neurotrauma, 2015. 32(24): p. 1968-80.

171. Caudle, K.L., et al., Hindlimb stretching alters locomotor function after spinal cord injury in the adult rat. Neurorehabil Neural Repair, 2015. 29(3): p. 268-77. 
172. Grau, J.W., et al., When Pain Hurts: Nociceptive Stimulation Induces a State of Maladaptive Plasticity and Impairs Recovery after Spinal Cord Injury. J Neurotrauma, 2016.

173. Murray, K.C., et al., Recovery of motoneuron and locomotor function after spinal cord injury depends on constitutive activity in 5-HT2C receptors. Nat Med, 2010. 16(6): p. 694-700.

174. Harvey, P.J., et al., Persistent sodium currents and repetitive firing in motoneurons of the sacrocaudal spinal cord of adult rats. J Neurophysiol, 2006. 96(3): p. 1141-57.

175. Fouad, K., et al., Locomotion after spinal cord injury depends on constitutive activity in serotonin receptors. J Neurophysiol, 2010. 104(6): p. 2975-84.

176. Rekling, J.C., et al., Synaptic control of motoneuronal excitability. Physiol Rev, 2000. 80(2): p. 767-852.

177. Jankowska, E. and I. Hammar, Spinal interneurones; how can studies in animals contribute to the understanding of spinal interneuronal systems in man? Brain Res Brain Res Rev, 2002. 40(1-3): p. 19-28.

178. Nielsen, J.B., C. Crone, and H. Hultborn, The spinal pathophysiology of spasticity-from a basic science point of view. Acta Physiol (Oxf), 2007. 189(2): p. 171-80.

179. Strommen, J.A., Management of spasticity from spinal cord dysfunction. Neurol Clin, 2013. 31(1): p. 269-86. 
180. Patrick, J.H., S.E. Farmer, and W. Bromwich, Muscle stretching for treatment and prevention of contracture in people with spinal cord injury. Spinal Cord, 2002. 40(8): p. 421-2; author reply 423.

181. Harvey, L.A., et al., Contracture management for people with spinal cord injuries. NeuroRehabilitation, 2011. 28(1): p. 17-20.

182. Nair, K.P. and J. Marsden, The management of spasticity in adults. Bmj, 2014. 349: p. g4737.

183. Goldspink, D.F., et al., Muscle growth in response to mechanical stimuli. Am J Physiol, 1995. 268(2 Pt 1): p. E288-97.

184. Cote, M.P., et al., Activity-dependent increase in neurotrophic factors is associated with an enhanced modulation of spinal reflexes after spinal cord injury. J Neurotrauma, 2011. 28(2): p. 299-309.

185. Magnuson, D.S., et al., Swimming as a model of task-specific locomotor retraining after spinal cord injury in the rat. Neurorehabil Neural Repair, 2009. 23(6): p. 535-45.

186. Caudle, K.L., et al., Hindlimb Stretching Alters Locomotor Function After Spinal Cord Injury in the Adult Rat. Neurorehabil Neural Repair, 2014.

187. Basso, D.M., M.S. Beattie, and J.C. Bresnahan, A sensitive and reliable locomotor rating scale for open field testing in rats. J Neurotrauma, 1995. 12(1): p. 1-21.

188. Kuerzi, J., et al., Task-specificity vs. ceiling effect: step-training in shallow water after spinal cord injury. Exp Neurol, 2010. 224(1): p. 178-87. 
189. Guissard, N., J. Duchateau, and K. Hainaut, Mechanisms of decreased motoneurone excitation during passive muscle stretching. Exp Brain Res, 2001. 137(2): p. 163-9.

190. Guissard, N. and J. Duchateau, Effect of static stretch training on neural and mechanical properties of the human plantar-flexor muscles. Muscle Nerve, 2004. 29(2): p. 248-55.

191. Magnuson, D.S., et al., Comparing deficits following excitotoxic and contusion injuries in the thoracic and lumbar spinal cord of the adult rat. Exp Neurol, 1999. 156(1): p. 191-204.

192. Jonkers, B.W., J.C. Sterk, and F.G. Wouterlood, Transcardial perfusion fixation of the CNS by means of a compressed-air-driven device. J Neurosci Methods, 1984. 12(2): p. 141-9.

193. Ceglia, L., et al., An evaluation of the reliability of muscle fiber cross-sectional area and fiber number measurements in rat skeletal muscle. Biol Proced Online, 2013. 15(1): p. 6.

194. Harvey, L., R. Herbert, and J. Crosbie, Does stretching induce lasting increases in joint ROM? A systematic review. Physiother Res Int, 2002. 7(1): p. 1-13.

195. Bodine-Fowler, S., Skeletal muscle regeneration after injury: an overview. J Voice, 1994. 8(1): p. 53-62.

196. Schultz, E., D.L. Jaryszak, and C.R. Valliere, Response of satellite cells to focal skeletal muscle injury. Muscle Nerve, 1985. 8(3): p. 217-22.

197. Minamoto, V.B., S.R. Bunho, and T.F. Salvini, Regenerated rat skeletal muscle after periodic contusions. Braz J Med Biol Res, 2001. 34(11): p. 1447-52. 
198. Stauber, W.T., et al., Fibrosis and intercellular collagen connections from four weeks of muscle strains. Muscle Nerve, 1996. 19(4): p. 423-30.

199. Castro, M.J., et al., Influence of complete spinal cord injury on skeletal muscle cross-sectional area within the first 6 months of injury. Eur J Appl Physiol Occup Physiol, 1999. 80(4): p. 373-8.

200. Zhong, H., et al., Differential modulation of myosin heavy chain phenotype in an inactive extensor and flexor muscle of adult rats. J Anat, 2007. 210(1): p. 19-31.

201. Mense, S. and H. Meyer, Different types of slowly conducting afferent units in cat skeletal muscle and tendon. J Physiol, 1985. 363: p. 403-17.

202. Cleland, C.L. and W.Z. Rymer, Neural mechanisms underlying the clasp-knife reflex in the cat. I. Characteristics of the reflex. J Neurophysiol, 1990. 64(4): p. 1303-18.

203. Cleland, C.L., L. Hayward, and W.Z. Rymer, Neural mechanisms underlying the clasp-knife reflex in the cat. II. Stretch-sensitive muscular-free nerve endings. J Neurophysiol, 1990. 64(4): p. 1319-30.

204. Gladwell, V.F. and J.H. Coote, Heart rate at the onset of muscle contraction and during passive muscle stretch in humans: a role for mechanoreceptors. J Physiol, 2002. 540(Pt 3): p. 1095-102.

205. Cleland, C.L., W.Z. Rymer, and F.R. Edwards, Force-sensitive interneurons in the spinal cord of the cat. Science, 1982. 217(4560): p. 652-5.

206. Amann, M., Significance of Group III and IV muscle afferents for the endurance exercising human. Clin Exp Pharmacol Physiol, 2012. 39(9): p. 831-5. 
207. Amann, M., et al., Implications of group III and IV muscle afferents for highintensity endurance exercise performance in humans. J Physiol, 2011. 589(Pt 21): p. 5299-309.

208. Ballermann, M. and K. Fouad, Spontaneous locomotor recovery in spinal cord injured rats is accompanied by anatomical plasticity of reticulospinal fibers. Eur J Neurosci, 2006. 23(8): p. 1988-96.

209. Wong, S.T., B.A. Atkinson, and L.C. Weaver, Confocal microscopic analysis reveals sprouting of primary afferent fibres in rat dorsal horn after spinal cord injury. Neurosci Lett, 2000. 296(2-3): p. 65-8.

210. Hagg, T., Collateral sprouting as a target for improved function after spinal cord injury. J Neurotrauma, 2006. 23(3-4): p. 281-94.

211. Harvey, L.A., et al., Quantifying the magnitude of torque physiotherapists apply when stretching the hamstring muscles of people with spinal cord injury. Arch Phys Med Rehabil, 2003. 84(7): p. 1072-5.

212. Hook, M.A., J.R. Huie, and J.W. Grau, Peripheral inflammation undermines the plasticity of the isolated spinal cord. Behav Neurosci, 2008. 122(1): p. 233-49.

213. Ferguson, A.R., et al., Maladaptive spinal plasticity opposes spinal learning and recovery in spinal cord injury. Front Physiol, 2012. 3: p. 399.

214. Smith, A.C., et al., Locomotor training alters the behavior of flexor reflexes during walking in human spinal cord injury. J Neurophysiol, 2014. 112(9): p. 2164-75.

215. Lovely, R.G., et al., Effects of training on the recovery of full-weight-bearing stepping in the adult spinal cat. Exp Neurol, 1986. 92(2): p. 421-35. 
216. Knikou, M. and C.K. Mummidisetty, Locomotor training improves premotoneuronal control after chronic spinal cord injury. J Neurophysiol, 2014. 111(11): p. 2264-75.

217. Dudley-Javoroski, S. and R.K. Shields, Assessment of physical function and secondary complications after complete spinal cord injury. Disabil Rehabil, 2006. 28(2): p. 103-10.

218. Keller, A.V., et al., Disruption of locomotion in response to hindlimb muscle stretch at acute and chronic time points after a spinal cord injury in rats. $\mathrm{J}$ Neurotrauma, 2016.

219. Cramer, J.T., et al., The acute effects of static stretching on peak torque, mean power output, electromyography, and mechanomyography. Eur J Appl Physiol, 2005. 93(5-6): p. 530-9.

220. Young, W. and S. Elliott, Acute effects of static stretching, proprioceptive neuromuscular facilitation stretching, and maximum voluntary contractions on explosive force production and jumping performance. Res Q Exerc Sport, 2001. 72(3): p. 273-9.

221. Cornwell, A., A.G. Nelson, and B. Sidaway, Acute effects of stretching on the neuromechanical properties of the triceps surae muscle complex. Eur J Appl Physiol, 2002. 86(5): p. 428-34.

222. Nelson, A.G., et al., Acute effects of passive muscle stretching on sprint performance. J Sports Sci, 2005. 23(5): p. 449-54. 
223. Nelson, A.G., J. Kokkonen, and D.A. Arnall, Acute muscle stretching inhibits muscle strength endurance performance. J Strength Cond Res, 2005. 19(2): $\mathrm{p}$. $338-43$.

224. Avela, J., H. Kyrolainen, and P.V. Komi, Altered reflex sensitivity after repeated and prolonged passive muscle stretching. J Appl Physiol (1985), 1999. 86(4): $\mathrm{p}$. 1283-91.

225. Nelson, A.G., et al., Inhibition of maximal voluntary isometric torque production by acute stretching is joint-angle specific. Res Q Exerc Sport, 2001. 72(1): p. 6870.

226. Cramer, J.T., et al., Acute effects of static stretching on peak torque in women. J Strength Cond Res, 2004. 18(2): p. 236-41.

227. Nelson, A.G., et al., Inhibition of maximal voluntary isokinetic torque production following stretching is velocity-specific. J Strength Cond Res, 2001. 15(2): p. 2416.

228. Hough, P.A., E.Z. Ross, and G. Howatson, Effects of dynamic and static stretching on vertical jump performance and electromyographic activity. $\mathrm{J}$ Strength Cond Res, 2009. 23(2): p. 507-12.

229. Beedle, B.B. and C.L. Mann, A comparison of two warm-ups on joint range of motion. J Strength Cond Res, 2007. 21(3): p. 776-9.

230. Taylor-Schroeder, S., et al., The SCIRehab project: treatment time spent in SCI rehabilitation. Physical therapy treatment time during inpatient spinal cord injury rehabilitation. J Spinal Cord Med, 2011. 34(2): p. 149-61. 
231. Koopmans, G.C., et al., The assessment of locomotor function in spinal cord injured rats: the importance of objective analysis of coordination. J Neurotrauma, 2005. 22(2): p. 214-25.

232. Rossi, A., R. Mazzocchio, and C. Scarpini, Clonus in man: a rhythmic oscillation maintained by a reflex mechanism. Electroencephalogr Clin Neurophysiol, 1990. 75(2): p. 56-63.

233. Beres-Jones, J.A., T.D. Johnson, and S.J. Harkema, Clonus after human spinal cord injury cannot be attributed solely to recurrent muscle-tendon stretch. Exp Brain Res, 2003. 149(2): p. 222-36.

234. Ishikawa, M. and P.V. Komi, Muscle fascicle and tendon behavior during human locomotion revisited. Exerc Sport Sci Rev, 2008. 36(4): p. 193-9.

235. Komi, P.V., Physiological and biomechanical correlates of muscle function: effects of muscle structure and stretch-shortening cycle on force and speed. Exerc Sport Sci Rev, 1984. 12: p. 81-121.

236. Walker, S., et al., Greater Strength Gains after Training with Accentuated Eccentric than Traditional Isoinertial Loads in Already Strength-Trained Men. Front Physiol, 2016. 7: p. 149.

237. Lynn, R. and D.L. Morgan, Decline running produces more sarcomeres in rat vastus intermedius muscle fibers than does incline running. J Appl Physiol (1985), 1994. 77(3): p. 1439-44.

238. Hedayatpour, N. and D. Falla, Physiological and Neural Adaptations to Eccentric Exercise: Mechanisms and Considerations for Training. Biomed Res Int, 2015. 2015: p. 193741. 
239. Armstrong, R.B., R.W. Ogilvie, and J.A. Schwane, Eccentric exercise-induced injury to rat skeletal muscle. J Appl Physiol Respir Environ Exerc Physiol, 1983. 54(1): p. 80-93.

240. Murase, S., et al., Bradykinin and nerve growth factor play pivotal roles in muscular mechanical hyperalgesia after exercise (delayed-onset muscle soreness). J Neurosci, 2010. 30(10): p. 3752-61.

241. Kniffki, K.D., E.D. Schomburg, and H. Steffens, Effects from fine muscle and cutaneous afferents on spinal locomotion in cats. J Physiol, 1981. 319: p. 543-54.

242. Schomburg, E.D., H. Steffens, and K.D. Kniffki, Contribution of group III and IV muscle afferents to multisensorial spinal motor control in cats. Neurosci Res, 1999. 33(3): p. 195-206.

243. Crameri, R.M., et al., Myofibre damage in human skeletal muscle: effects of electrical stimulation versus voluntary contraction. J Physiol, 2007. 583(Pt 1): $\mathrm{p}$. $365-80$.

244. Prasartwuth, O., J.L. Taylor, and S.C. Gandevia, Maximal force, voluntary activation and muscle soreness after eccentric damage to human elbow flexor muscles. J Physiol, 2005. 567(Pt 1): p. 337-48.

245. Vila-Cha, C., et al., Eccentric exercise and delayed onset muscle soreness of the quadriceps induce adjustments in agonist-antagonist activity, which are dependent on the motor task. Exp Brain Res, 2012. 216(3): p. 385-95.

246. Degtyarenko, A.M. and M.P. Kaufman, Bicuculline and strychnine suppress the mesencephalic locomotor region-induced inhibition of group III muscle afferent input to the dorsal horn. Neuroscience, 2003. 118(3): p. 779-88. 
247. Detloff, M.R., et al., Delayed Exercise Is Ineffective at Reversing Aberrant Nociceptive Afferent Plasticity or Neuropathic Pain After Spinal Cord Injury in Rats. Neurorehabil Neural Repair, 2016. 30(7): p. 685-700.

248. Walters, E.T., Nociceptors as chronic drivers of pain and hyperreflexia after spinal cord injury: an adaptive-maladaptive hyperfunctional state hypothesis. Front Physiol, 2012. 3: p. 309.

249. Ramer, L.M., et al., Plasticity of TRPVI-Expressing Sensory Neurons Mediating Autonomic Dysreflexia Following Spinal Cord Injury. Front Physiol, 2012. 3: p. 257.

250. Grau, J.W., et al., Metaplasticity and behavior: how training and inflammation affect plastic potential within the spinal cord and recovery after injury. Front Neural Circuits, 2014. 8: p. 100.

251. Bouffard, J., et al., Tonic pain experienced during locomotor training impairs retention despite normal performance during acquisition. J Neurosci, 2014. 34(28): p. 9190-5.

252. Avela, J., et al., Neural and mechanical responses of the triceps surae muscle group after 1 h of repeated fast passive stretches. J Appl Physiol (1985), 2004. 96(6): p. 2325-32.

253. Cramer, J.T., et al., Acute effects of static stretching on maximal eccentric torque production in women. J Strength Cond Res, 2006. 20(2): p. 354-8.

254. Keller, A.V., et al., Dynamic "range of motion" hindlimb stretching disrupts locomotor function in rats with moderate subacute spinal cord injuries. $\mathrm{J}$ Neurotrauma, 2017. 
255. Agarwal, G.C. and G.L. Gottlieb, Oscillation of the human ankle joint in response to applied sinusoidal torque on the foot. J Physiol, 1977. 268(1): p. 151-76.

256. Wallace, D.M., B.H. Ross, and C.K. Thomas, Characteristics of lower extremity clonus after human cervical spinal cord injury. J Neurotrauma, 2012. 29(5): p. 915-24.

257. Adams, M.M. and A.L. Hicks, Spasticity after spinal cord injury. Spinal Cord, 2005. 43(10): p. 577-86.

258. Hultborn, H. and J. Malmsten, Changes in segmental reflexes following chronic spinal cord hemisection in the cat. I. Increased monosynaptic and polysynaptic ventral root discharges. Acta Physiol Scand, 1983. 119(4): p. 405-22.

259. Bose, P., R. Parmer, and F.J. Thompson, Velocity-dependent ankle torque in rats after contusion injury of the midthoracic spinal cord: time course. J Neurotrauma, 2002. 19(10): p. 1231-49.

260. Bennett, D.J., et al., Spasticity in rats with sacral spinal cord injury. J Neurotrauma, 1999. 16(1): p. 69-84.

261. van Gorp, S., et al., Translation of the rat thoracic contusion model; part 1supraspinally versus spinally mediated pain-like responses and spasticity. Spinal Cord, 2014. 52(7): p. 524-8.

262. Hagbarth, K.E., et al., Muscle spindle activity in alternating tremor of Parkinsonism and in clonus. J Neurol Neurosurg Psychiatry, 1975. 38(7): p. 63641. 
263. Moriyama, H., et al., Comparison of muscular and articular factors in the progression of contractures after spinal cord injury in rats. Spinal Cord, 2006. 44(3): p. 174-81.

264. Schmalbruch, H., Fiber composition of the rat sciatic nerve. Anat Rec, 1986. 215(1): p. 71-81.

265. Stacey, M.J., Free nerve endings in skeletal muscle of the cat. J Anat, 1969. 105(Pt 2): p. 231-54.

266. Amann, M., et al., Peripheral fatigue limits endurance exercise via a sensory feedback-mediated reduction in spinal motoneuronal output. J Appl Physiol (1985), 2013. 115(3): p. 355-64.

267. Nagy, J.I., et al., Biochemical and anatomical observations on the degeneration of peptide-containing primary afferent neurons after neonatal capsaicin. Neuroscience, 1981. 6(10): p. 1923-34.

268. Nagy, J.I., et al., Dose-dependent effects of capsaicin on primary sensory neurons in the neonatal rat. J Neurosci, 1983. 3(2): p. 399-406.

269. D'Amour, F.E.A.S., DONN L., A METHOD FOR DETERMINING LOSS OF PAIN SENSATION. Journal of Pharmacology and Experimental Therapeutics 1941. 72(1): p. 74-79.

270. Petruska, J.C., et al., Organization of sensory input to the nociceptive-specific cutaneous trunk muscle reflex in rat, an effective experimental system for examining nociception and plasticity. J Comp Neurol, 2014. 522(5): p. 1048-71.

271. Hsu, S.M., L. Raine, and H. Fanger, Use of avidin-biotin-peroxidase complex $(A B C)$ in immunoperoxidase techniques: a comparison between $A B C$ and 
unlabeled antibody (PAP) procedures. J Histochem Cytochem, 1981. 29(4): $\mathrm{p}$. $577-80$.

272. Jancso, G., et al., Neurotoxic effect of capsaicin in mammals. Acta Physiol Hung, 1987. 69(3-4): p. 295-313.

273. Zinck, N.D., V.F. Rafuse, and J.W. Downie, Sprouting of CGRP primary afferents in lumbosacral spinal cord precedes emergence of bladder activity after spinal injury. Exp Neurol, 2007. 204(2): p. 777-90.

274. Armstrong, R.B., Mechanisms of exercise-induced delayed onset muscular soreness: a brief review. Med Sci Sports Exerc, 1984. 16(6): p. 529-38.

275. Ota, H., et al., TRPVI and TRPV4 play pivotal roles in delayed onset muscle soreness. PLoS One, 2013. 8(6): p. e65751.

276. Kubo, A., et al., Absence of mechanical hyperalgesia after exercise (delayed onset muscle soreness) in neonatally capsaicin-treated rats. Neurosci Res, 2012. 73(1): p. $56-60$.

277. Zytnicki, D., et al., Reduction of Ib autogenetic inhibition in motoneurons during contractions of an ankle extensor muscle in the cat. J Neurophysiol, 1990. 64(5): p. $1380-9$.

278. Jankowska, E., Interneuronal relay in spinal pathways from proprioceptors. Prog Neurobiol, 1992. 38(4): p. 335-78.

279. Delwaide, P.J. and E. Oliver, Short-latency autogenic inhibition (IB inhibition) in human spasticity. J Neurol Neurosurg Psychiatry, 1988. 51(12): p. 1546-50.

280. Dubin, A.E. and A. Patapoutian, Nociceptors: the sensors of the pain pathway. $\mathrm{J}$ Clin Invest, 2010. 120(11): p. 3760-72. 
281. Kalous, A., P.B. Osborne, and J.R. Keast, Acute and chronic changes in dorsal horn innervation by primary afferents and descending supraspinal pathways after spinal cord injury. J Comp Neurol, 2007. 504(3): p. 238-53.

282. Hou, S., H. Duale, and A.G. Rabchevsky, Intraspinal sprouting of unmyelinated pelvic afferents after complete spinal cord injury is correlated with autonomic dysreflexia induced by visceral pain. Neuroscience, 2009. 159(1): p. 369-79.

283. Nishigami, T., et al., Changes in calcitonin gene-related peptide expression following joint immobilization in rats. Neurosci Lett, 2009. 454(1): p. 97-100.

284. Behm, D.G., et al., Acute effects of muscle stretching on physical performance, range of motion, and injury incidence in healthy active individuals: a systematic review. Appl Physiol Nutr Metab, 2016. 41(1): p. 1-11.

285. Lundeberg, T., Pain physiology and principles of treatment. Scand J Rehabil Med Suppl, 1995. 32: p. 13-41.

286. Schomburg, E.D., H. Steffens, and N. Wada, Parallel nociceptive reflex pathways with negative and positive feedback functions to foot extensors in the cat. $\mathrm{J}$ Physiol, 2001. 536(Pt 2): p. 605-13.

287. Blivis, D., et al., Differential effects of opioids on sacrocaudal afferent pathways and central pattern generators in the neonatal rat spinal cord. J Neurophysiol, 2007. 97(4): p. 2875-86.

288. Cherniak, M., et al., The sacral networks and neural pathways used to elicit lumbar motor rhythm in the rodent spinal cord. Front Neural Circuits, 2014. 8: p. 143. 
289. Etlin, A., et al., Characterization of sacral interneurons that mediate activation of locomotor pattern generators by sacrocaudal afferent input. J Neurosci, 2013. 33(2): p. 734-47.

290. Mandadi, S., et al., Identification of multisegmental nociceptive afferents that modulate locomotor circuits in the neonatal mouse spinal cord. J Comp Neurol, 2013. 521(12): p. 2870-87.

291. Mandadi, S., et al., Locomotor networks are targets of modulation by sensory transient receptor potential vanilloid 1 and transient receptor potential melastatin 8 channels. Neuroscience, 2009. 162(4): p. 1377-97.

292. Gomez-Pinilla, F., et al., BDNF and learning: Evidence that instrumental training promotes learning within the spinal cord by up-regulating BDNF expression. Neuroscience, 2007. 148(4): p. 893-906.

293. Ferguson, A.R., et al., Cell death after spinal cord injury is exacerbated by rapid TNF alpha-induced trafficking of GluR2-lacking AMPARs to the plasma membrane. J Neurosci, 2008. 28(44): p. 11391-400.

294. Baumbauer, K.M., et al., Timing in the absence of supraspinal input I: variable, but not fixed, spaced stimulation of the sciatic nerve undermines spinallymediated instrumental learning. Neuroscience, 2008. 155(4): p. 1030-47.

295. Ferguson, A.R., et al., Group I metabotropic glutamate receptors control metaplasticity of spinal cord learning through a protein kinase C-dependent mechanism. J Neurosci, 2008. 28(46): p. 11939-49. 
296. Patton, B.C., et al., The behavioral deficit observed following noncontingent shock in spinalized rats is prevented by the protein synthesis inhibitor cycloheximide. Behav Neurosci, 2004. 118(3): p. 653-8.

297. Baumbauer, K.M., et al., Intrathecal infusions of anisomycin impact the learning deficit but not the learning effect observed in spinal rats that have received instrumental training. Behav Brain Res, 2006. 173(2): p. 299-309.

298. Huie, J.R., et al., Glial tumor necrosis factor alpha (TNFalpha) generates metaplastic inhibition of spinal learning. PLoS One, 2012. 7(6): p. e39751.

299. Watkins, L.R., et al., Evidence for the involvement of spinal cord glia in subcutaneous formalin induced hyperalgesia in the rat. Pain, 1997. 71(3): p. 22535.

300. Liu, Y.L., et al., Tumor necrosis factor-alpha induces long-term potentiation of C-fiber evoked field potentials in spinal dorsal horn in rats with nerve injury: the role of NF-kappa B, JNK and p38 MAPK. Neuropharmacology, 2007. 52(3): p. 708-15.

301. Choi, J.I., et al., Peripheral inflammation induces tumor necrosis factor dependent AMPA receptor trafficking and Akt phosphorylation in spinal cord in addition to pain behavior. Pain, 2010. 149(2): p. 243-53.

302. Joynes, R.L., et al., Instrumental learning within the spinal cord: V. Evidence the behavioral deficit observed after noncontingent nociceptive stimulation reflects an intraspinal modification. Behav Brain Res, 2003. 141(2): p. 159-70.

303. Abraham, W.C., Metaplasticity: tuning synapses and networks for plasticity. Nat Rev Neurosci, 2008. 9(5): p. 387. 
304. Schomburg, E.D. and H. Steffens, Influence of opioids and naloxone on rhythmic motor activity in spinal cats. Exp Brain Res, 1995. 103(3): p. 333-43.

305. Faden, A.I., Opioid and nonopioid mechanisms may contribute to dynorphin's pathophysiological actions in spinal cord injury. Ann Neurol, 1990. 27(1): p. 6774.

306. Hook, M.A., et al., Neurobiological Effects of Morphine after Spinal Cord Injury. J Neurotrauma, 2017. 34(3): p. 632-644.

307. Turtle, J.D., et al., Pain Input Impairs Recovery after Spinal Cord Injury: Treatment with Lidocaine. J Neurotrauma, 2017. 34(6): p. 1200-1208.

308. Woller, S.A. and M.A. Hook, Opioid administration following spinal cord injury: implications for pain and locomotor recovery. Exp Neurol, 2013. 247: p. 328-41.

309. Ossipov, M.H., G.O. Dussor, and F. Porreca, Central modulation of pain. J Clin Invest, 2010. 120(11): p. 3779-87.

310. Marvizon, J.C., W. Chen, and N. Murphy, Enkephalins, dynorphins, and betaendorphin in the rat dorsal horn: an immunofluorescence colocalization study. $\mathrm{J}$ Comp Neurol, 2009. 517(1): p. 51-68.

311. Tuchscherer, M.M. and V.S. Seybold, A quantitative study of the coexistence of peptides in varicosities within the superficial laminae of the dorsal horn of the rat spinal cord. J Neurosci, 1989. 9(1): p. 195-205.

312. Basbaum, A.I., L. Cruz, and E. Weber, Immunoreactive dynorphin B in sacral primary afferent fibers of the cat. J Neurosci, 1986. 6(1): p. 127-33. 
313. Iadarola, M.J., et al., Enhancement of dynorphin gene expression in spinal cord following experimental inflammation: stimulus specificity, behavioral parameters and opioid receptor binding. Pain, 1988. 35(3): p. 313-26.

314. Kajander, K.C., et al., Dynorphin increases in the dorsal spinal cord in rats with a painful peripheral neuropathy. Peptides, 1990. 11(4): p. 719-28.

315. Noguchi, K., et al., Dynorphin expression and Fos-like immunoreactivity following inflammation induced hyperalgesia are colocalized in spinal cord neurons. Brain Res Mol Brain Res, 1991. 10(3): p. 227-33.

316. Abraham, K.E., J.F. McGinty, and K.L. Brewer, The role of kainic acid/AMPA and metabotropic glutamate receptors in the regulation of opioid $m R N A$ expression and the onset of pain-related behavior following excitotoxic spinal cord injury. Neuroscience, 2001. 104(3): p. 863-74.

317. Faden, A.I., et al., Increased dynorphin immunoreactivity in spinal cord after traumatic injury. Regul Pept, 1985. 11(1): p. 35-41.

318. Luo, M.C., et al., Spinal dynorphin and bradykinin receptors maintain inflammatory hyperalgesia. J Pain, 2008. 9(12): p. 1096-105.

319. Walker, J.M., et al., Dynorphin (1-17): lack of analgesia but evidence for nonopiate electrophysiological and motor effects. Life Sci, 1982. 31(16-17): p. 18214.

320. Xu, M., et al., Neuropathic pain activates the endogenous kappa opioid system in mouse spinal cord and induces opioid receptor tolerance. J Neurosci, 2004. 24(19): p. 4576-84. 
321. Bakshi, R., A.H. Newman, and A.I. Faden, Dynorphin A-(1-17) induces alterations in free fatty acids, excitatory amino acids, and motor function through an opiate-receptor-mediated mechanism. J Neurosci, 1990. 10(12): p. 3793-800.

322. Thor, K.B., et al., Plasticity in spinal opioid control of lower urinary tract function in paraplegic cats. Neuroreport, 1994. 5(13): p. 1673-8.

323. Craig, A.D., Distribution of brainstem projections from spinal lamina I neurons in the cat and the monkey. J Comp Neurol, 1995. 361(2): p. 225-48.

324. Amann, M., et al., On the contribution of group III and IV muscle afferents to the circulatory response to rhythmic exercise in humans. J Physiol, 2011. 589(Pt 15): p. $3855-66$.

325. Sidhu, S.K., et al., Group III/IV locomotor muscle afferents alter motor cortical and corticospinal excitability and promote central fatigue during cycling exercise. Clin Neurophysiol, 2017. 128(1): p. 44-55.

326. Pettorossi, V.E., et al., The role of capsaicin-sensitive muscle afferents in fatigueinduced modulation of the monosynaptic reflex in the rat. J Physiol, 1999. 515 ( Pt 2): p. 599-607.

327. Yang, J.F., R.B. Stein, and K.B. James, Contribution of peripheral afferents to the activation of the soleus muscle during walking in humans. Exp Brain Res, 1991. 87(3): p. 679-87.

328. Biering-Sorensen, B., et al., Muscle after spinal cord injury. Muscle Nerve, 2009. 40(4): p. 499-519.

329. Igawa, Y., et al., The role of capsaicin-sensitive afferents in autonomic dysreflexia in patients with spinal cord injury. BJU Int, 2003. 91(7): p. 637-41. 
330. Cragg, J. and A. Krassioukov, Autonomic dysreflexia. Cmaj, 2012. 184(1): p. 66.

331. De Jaeger, D., V. Joumaa, and W. Herzog, Intermittent stretch training of rabbit plantarflexor muscles increases soleus mass and serial sarcomere number. J Appl Physiol (1985), 2015. 118(12): p. 1467-73.

332. Chleboun, G.S., et al., In vivo measurement of fascicle length and pennation angle of the human biceps femoris muscle. Cells Tissues Organs, 2001. 169(4): p. 4019.

333. Baewer, D.V., et al., Passive stretch inhibits central corelike lesion formation in the soleus muscles of hindlimb-suspended unloaded rats. J Appl Physiol (1985), 2004. 97(3): p. 930-4.

334. Van Dyke, J.M., J.L. Bain, and D.A. Riley, Preserving sarcomere number after tenotomy requires stretch and contraction. Muscle Nerve, 2012. 45(3): p. 367-75.

335. Webright, W.G., B.J. Randolph, and D.H. Perrin, Comparison of nonballistic active knee extension in neural slump position and static stretch techniques on hamstring flexibility. J Orthop Sports Phys Ther, 1997. 26(1): p. 7-13.

336. de Weijer, V.C., G.C. Gorniak, and E. Shamus, The effect of static stretch and warm-up exercise on hamstring length over the course of 24 hours. J Orthop Sports Phys Ther, 2003. 33(12): p. 727-33.

337. Chan, S.P., Y. Hong, and P.D. Robinson, Flexibility and passive resistance of the hamstrings of young adults using two different static stretching protocols. Scand J Med Sci Sports, 2001. 11(2): p. 81-6. 
338. Spernoga, S.G., et al., Duration of Maintained Hamstring Flexibility After a OneTime, Modified Hold-Relax Stretching Protocol. J Athl Train, 2001. 36(1): p. 4448.

339. Weppler, C.H. and S.P. Magnusson, Increasing muscle extensibility: a matter of increasing length or modifying sensation? Phys Ther, 2010. 90(3): p. 438-49.

340. Funk, D.C., et al., Impact of prior exercise on hamstring flexibility: a comparison of proprioceptive neuromuscular facilitation and static stretching. J Strength Cond Res, 2003. 17(3): p. 489-92.

341. Etnyre, B.R. and L.D. Abraham, Gains in range of ankle dorsiflexion using three popular stretching techniques. Am J Phys Med, 1986. 65(4): p. 189-96.

342. Wallin, D., et al., Improvement of muscle flexibility. A comparison between two techniques. Am J Sports Med, 1985. 13(4): p. 263-8.

343. Sharman, M.J., A.G. Cresswell, and S. Riek, Proprioceptive neuromuscular facilitation stretching : mechanisms and clinical implications. Sports Med, 2006. 36(11): p. 929-39.

344. Smith, J.L., R.S. Hutton, and E. Eldred, Postcontraction changes in sensitivity of muscle afferents to static and dynamic stretch. Brain Res, 1974. 78(2): p. 193202.

345. Edin, B.B. and A.B. Vallbo, Muscle afferent responses to isometric contractions and relaxations in humans. J Neurophysiol, 1990. 63(6): p. 1307-13.

346. Magnusson, S.P., Passive properties of human skeletal muscle during stretch maneuvers. A review. Scand J Med Sci Sports, 1998. 8(2): p. 65-77. 
347. Chalmers, G., Re-examination of the possible role of Golgi tendon organ and muscle spindle reflexes in proprioceptive neuromuscular facilitation muscle stretching. Sports Biomech, 2004. 3(1): p. 159-83.

348. Magnusson, S.P., et al., A mechanism for altered flexibility in human skeletal muscle. J Physiol, 1996. 497 (Pt 1): p. 291-8.

349. Magnusson, S.P., et al., Mechanical and physical responses to stretching with and without preisometric contraction in human skeletal muscle. Arch Phys Med Rehabil, 1996. 77(4): p. 373-8.

350. Magnusson, S.P., et al., Determinants of musculoskeletal flexibility: viscoelastic properties, cross-sectional area, EMG and stretch tolerance. Scand J Med Sci Sports, 1997. 7(4): p. 195-202.

351. Gajdosik, R.L., D.W. Vander Linden, and A.K. Williams, Influence of age on length and passive elastic stiffness characteristics of the calf muscle-tendon unit of women. Phys Ther, 1999. 79(9): p. 827-38.

352. Feland, J.B., et al., The effect of duration of stretching of the hamstring muscle group for increasing range of motion in people aged 65 years or older. Phys Ther, 2001. 81(5): p. 1110-7.

353. Kokkonen, J., A.G. Nelson, and A. Cornwell, Acute muscle stretching inhibits maximal strength performance. Res Q Exerc Sport, 1998. 69(4): p. 411-5.

354. Taylor, D.C., et al., Viscoelastic properties of muscle-tendon units. The biomechanical effects of stretching. Am J Sports Med, 1990. 18(3): p. 300-9. 
355. Chaouachi, A., et al., Unilateral static and dynamic hamstrings stretching increases contralateral hip flexion range of motion. Clin Physiol Funct Imaging, 2017. 37(1): p. 23-29.

356. Della Torre, G., O. Brunetti, and V.E. Pettorossi, Capsaicin-sensitive muscle afferents modulate the monosynaptic reflex in response to muscle ischemia and fatigue in the rat. Arch Ital Biol, 2002. 140(1): p. 51-65.

357. Suzuki, T., et al., Effect of continued stretching of the affected arm in patients with cerebrovascular diseases by examining H-reflex characteristics. Electromyogr Clin Neurophysiol, 2003. 43(1): p. 51-6.

358. Chung, S., et al., Changes of Reflex, Non-reflex and Torque Generation Properties of Spastic Ankle Plantar Flexors Induced by Intelligent Stretching. Conf Proc IEEE Eng Med Biol Soc, 2005. 4: p. 3672-5.

359. Bakheit, A.M., V. Maynard, and S. Shaw, The effects of isotonic and isokinetic muscle stretch on the excitability of the spinal alpha motor neurones in patients with muscle spasticity. Eur J Neurol, 2005. 12(9): p. 719-24.

360. Li, W.W., et al., Substance P spinal signaling induces glial activation and nociceptive sensitization after fracture. Neuroscience, 2015. 310: p. 73-90. 


\section{LIST OF ABBREVIATIONS AND SYMBOLS}

5-HT

AcS

$\mathrm{AD}$

ADL

AMPA

ASIA

ATP

BBB

BDNF

$\mathrm{BF}$

BSA

BWSTT

CaMKII

cAMP

CAP

$\mathrm{CC}$

CGRP

$\mathrm{ChS}$

$\mathrm{CN}$

CNS

$\mathrm{CON}$

$\mathrm{CPG}$

CPI

CREB

CSA

CTMR

CV

DAMP

DF

$\mathrm{DH}$

DNA

DOMS

EC

EMG

ES

FMA

GABA

GluA2 5-hydroxytryptamine

acute stretch

autonomic dysreflexia

activities of daily living

alpha-amino-3-hydroxy-5-methyl-isoxazolepropionate

American Spinal Injury Association

adenosine triphosphate

Basso, Beattie, Bresnahan (Open Field Locomotor Scale)

brain derived neurotrophic factor

Biceps Femoris

bovine serum albumin

body weight supported treadmill training

calcium/calmodulin activated kinase II

cyclic adenosine monophosphate

capsaicin (group)

central canal

calcitonin gene related peptide

chronic stretch

centralized nuclei

central nervous system

control (group)

central pattern generator

central pattern index

cAMP response element binding protein

cross sectional area

cutaneous trunci muscle reflex

cardiovascular

damage-associated molecular patterns

degrees of freedom

dorsal horn

deoxyribonucleic acid

delayed onset muscle soreness

Eriochrome cyanine

electromyography

electrical stimulation

Fugl-Meyer assessment

gamma-Aminobutyric acid

glutamate A2 (subunit) 


\begin{tabular}{|c|c|}
\hline GM & gastrocnemius \\
\hline GTO & golgi tendon organ \\
\hline $\mathrm{H} \& \mathrm{E}$ & hematoxylin and eosin \\
\hline HAT & hip-ankle-toe \\
\hline H-reflex & Hoffman-reflex \\
\hline IGM & intermediate gray matter \\
\hline IHA & iliac crest-hip-ankle \\
\hline $\mathrm{IHC}$ & immunohistochemistry \\
\hline IL & interleukin \\
\hline IS & index finger sensor \\
\hline KAT & knee-ankle-toe \\
\hline $\mathrm{KCC} 2$ & potassium-chloride exchanger \\
\hline KOR & kappa receptor \\
\hline L1-L5 & lumbar level 1-5 \\
\hline L-DOPA & L-3,4-dihydroxyphenylalanine \\
\hline LTP & long term potentiation \\
\hline MAS & Modified Ashworth Scale \\
\hline MEMP & magnetically evoked muscle potential \\
\hline MEMR & magnetically evoked muscle response \\
\hline MF & muscle fiber \\
\hline mGluR & metabotropic glutamate receptor \\
\hline MLR & mesencephalic locomotor region \\
\hline NDS & normal donkey serum \\
\hline $\mathrm{NIH}$ & national institute of health \\
\hline NK1R & neurokinin 1 receptor \\
\hline NMDA & N-methyl-D-aspartate \\
\hline NRM & nucleus raphe magnus \\
\hline NSCISC & National Spinal Cord Injury Statistical Center \\
\hline NYU & New York University (impactor) \\
\hline $\mathrm{PBS} / \mathrm{T}$ & phosphate buffered saline/triton \\
\hline $\mathrm{PF}$ & pattern formation \\
\hline PFA & paraformaldehyde \\
\hline PI3K & phosphoinositide 3-kinase \\
\hline PIC & persistent inward current \\
\hline $\mathrm{PKC}$ & protein kinase $\mathrm{C}$ \\
\hline PNF & proprioceptive neuromuscular facilitation \\
\hline PPN & pedunculopontine nucleus \\
\hline PSI & plantar stepping index \\
\hline PT & physical therapy/therapist \\
\hline RG & reticularis gigantocellularis \\
\hline $\mathrm{RG}$ & rhythm generation \\
\hline RI & regularity index \\
\hline RM ANOVA & repeated measure analysis of variance \\
\hline ROM & range of motion \\
\hline S4 & sacral level 4 \\
\hline SCA & sacracaudle afferents \\
\hline
\end{tabular}




\begin{tabular}{ll} 
SCI & spinal cord injury \\
SD & Sprague Dawley \\
SD & Standard deviation \\
SG & substantia gelatanosa \\
SWM & spared white matter \\
T10 & thoracic level 10 \\
TA & Tibialis Anterior \\
tES & transcutaneous electrical stimulation \\
TNF- $\alpha$ & tumor necrosis factor alpha \\
TRPV1 & Transient Receptor Potential Vanilloid 1 \\
TS & thumb sensor \\
VEH & vehicle (group) \\
VH & ventral horn \\
$\mu \mathrm{m}$ & micrometer \\
$\mathrm{cm}$ & centimeter \\
$\mathrm{g}$ & gram \\
$\mathrm{kg}$ & kilogram \\
$\mathrm{m}$ & meter \\
$\mathrm{mg}$ & milligram \\
$\mathrm{mm}$ & millimeter \\
$\mathrm{N} * \mathrm{~m} / \mathrm{cm}$ & Newton meter/centimeter \\
$\mathrm{s}$ & second \\
$\mathrm{v} / \mathrm{v}$ & volume-to-volume \\
& \\
\hline &
\end{tabular}




\title{
CURRICULUM VITAE
}

\author{
Anastasia Keller \\ 511 South Floyd Street \\ Louisville, KY 40204 \\ 5027446200 \\ avprok01@louisville.edu
}

\section{Education}

PhD Student

June 2017

University of Louisville, Louisville $K Y$

Department of Physiology and Biophysics

Master of Science with Honors for Excellence

May 2012

University of Louisville, Louisville $K Y$

Exercise Physiology

Bachelor of Science with Honors for Excellence

May 2010

University of Louisville, Louisville, $K Y$

Health and Human Performance with a concentration in Exercise Science

High School Degree

June 2006

Lyceum of Humanities, Pskov, Russia

Linguistics

\section{Professional Memberships and Activities:}

- Kentucky Spinal Cord Injury Research Center (January 2013-present)

- National Neurotrauma Society (June 2013-present)

- Society for Neuroscience (October 2014-present)

- Graduate Student Council: representative for the Department of Physiology (August 2014-present) 


\section{$\underline{\text { Scientific Publications: }}$}

- Keller AV, Rees KM, Prince DC, Morehouse JR, Shum-Siu A, Magnuson DS. Dynamic "range of motion" hindlimb stretching disrupts locomotor function in rats with moderate subacute spinal cord injuries. J. Neurotrauma 2017 (March, online ahead of print)

- Keller AV, Wainwright G, Shum-Siu A, Prince D, Hoeper A, Martin E, Magnuson DS. Disruption of Locomotion in Response to Hindlimb Muscle Stretch at Acute and Chronic Time Points after a Spinal Cord Injury in Rats. J Neurotrauma. 2017 (February)

- Keller AV, Rees KM, Morehouse JR, Wade A, Shum-Siu A, Magnuson DS. Electromyographic patterns of the hindlimb knee muscles in response to muscle stretch in rats with spinal cord injuries. (in preparation)

- Keller AV, Hainline CL, Rees KM, Prince DC, Lucas S, Shum-Siu A, Petruska, JC, Magnuson DS. Role of nociceptive afferents in stretch-induced locomotor deficits in rats with spinal cord injuries. (in preparation)

\section{$\underline{\text { Abstracts: }}$}

- Society for Neuroscience: 1. Stretching disrupts locomotor function in rats with spinal cord injuries: Static "stretch and hold" vs dynamic "range of motion" patterns. 2. Stretching disrupts locomotor function in rats with spinal cord injuries: role of nociceptive afferents. (November 2016)

- Research! Louisville: Involvement of Capsaicin Sensitive Nociceptive Afferents in Stretch-Induced Locomotor Deficits in Rats with SCI (September 2016)

- Neuroscience Day: Involvement of Capsaicin Sensitive Nociceptive Afferents in StretchInduced Locomotor Deficits in Rats with SCI (April 2016)

- Society for Neuroscience: Electromyographic patterns of the contralateral limb in response to muscle stretch in rats with mild spinal cord injuries (October 2015)

- ASIA and ISCoS joint scientific meeting: Electromyographic patterns of the contralateral limb in response to muscle stretch in rats with mild spinal cord injuries (May 2015)

- Satellite Symposia on Spinal Cord Plasticity in Motor Control Symposium: Hindlimb muscle stretch reduces locomotor function after a spinal cord injury: Implications for Physical Therapy and Rehabilitation (September 2014) 
- Research! Louisville: Hindlimb muscle stretch reduces locomotor function after a Spinal Cord Injury: Implications for Physical Therapy and Rehabilitation (September 2014)

- National Neurotrauma Symposium: Hindlimb muscle stretch reduces locomotor function after a Spinal Cord Injury: Implications for Physical Therapy and Rehabilitation (June 2014)

- $15^{\text {th }}$ International Symposium on Neural Regeneration: Hindlimb Muscle Stretch Reduces Locomotor Function after a Spinal Cord Injury. Acute and Chronic Time Points (December 2013)

- Research! Louisville: "Hindlimb Muscle Stretch Reduces Locomotor Function after a Spinal Cord Injury: Implications for Physical Therapy and Rehabilitation" (September 2013)

- Research! Louisville: Force sensing glove for quantification of joint torques during stretching after spinal cord injury in the rat model, $2^{\text {nd }}$ author (June 2013)

\section{Presentations:}

- KSCIRC seminar series: Involvement of Capsaicin Sensitive Nociceptive Afferents in Stretch-Induced Locomotor Deficits in Rats with SCI (May, 2016)

- KSCIRC Journal Club: Trunk Robot Rehabilitation Training with Active Training Reorganizes and Enriches Trunk Motor Cortex Representations in Spinal Transected Rats (Oza and Giszter, 2015) (October, 2015)

- KSCIRC seminar series: Identification of EMG patterns in the contralateral limb in response to muscle stretch in rats with mild SCIs (May, 2015)

- KSCIRC Journal Club: Wnt7a stimulates myogenic stem cell motility and engraftment resulting in improved muscle strength, (Bentzinger, et al 2014) (October 2014)

- KSCIRC seminar series: Vulnerability of the Central Pattern Generation to Muscle Stretch after a Spinal Cord Injury (June 2014)

- KSCIRC Journal Club: Pattern of Innervation and Recruitment of Different Classes of Motoneurons in Adult Zebrafish, (Ampatzis et al, 2013; J.Neuroscience) (April 2014)

\section{Scientific Meetings Attended:}

- Society for Neuroscience Conference (October 2015, Chicago) 
- ASIA and ISCoS joint Scientific Meeting (May 2015, Montreal, Canada)

- Society for Neuroscience Conference (November 2014, Washington DC)

- Satellite Symposium on Spinal Cord Plasticity in Motor Control

- National Neurotrauma Symposium (August 2013, Nashville, TN; June 2014, San Francisco, CA)

- Kentucky Spinal Cord and Head Injury Research Symposium (June 2013, Louisville KY; May 2014, Lexington, KY; May 2015; Louisville KY, May 2016, Lexington, KY)

- Neuroscience Day (April 2013, 2014, University of Louisville)

- $15^{\text {th }}$ International Symposium on Neural Regeneration (December 2013, Pacific Grove, CA)

- Research! Louisville (September 2012, 2013, 2014, University of Louisville)

\section{$\underline{\text { Scientific Awards: }}$}

- Research! Louisville $1^{\text {st }}$ place Student Engineering Collaboration Award (September, 2014)

- National Neurotrauma Society Travel Award Recipient (June 2014)

- Neuroscience Day ${ }^{\text {rd }}$ place Award Winner (April 2014)

- International Symposium on Neural Regeneration Travel Award Recipient (December, 2013)

- Research! Louisville $2^{\text {nd }}$ place Award Winner (June 2013)

\section{Internship/Work Experience:}

- Division I Women's Rowing Graduate Assistant Coach (August 2011 - May 2012)

- University of Louisville Division I Athletics - Strength and Conditioning Coach Assistant (February-April 2010) 


\section{Other Academic and Athletic Achievements:}

- NCAA Division I Rowing, Full Athletic Scholarship, Team Captain (2009-2011)

- All Big East First Team Selection Award (2011)

- Big East Champion in the Varsity Eight Event $(2009,2011)$

- IronCardinal (2009-2011)

- Big East Academic All-Star $(2008,2009)$

- Dean's List $(2007,2008,2009)$

- Athletic Director's Honor Roll $(2008,2009)$

- Red and Black Scholar (2006, 2007, 2008, 2009)

- CRCA National Scholar Athlete (2009)

- Silver medalist in the U-18 at the Russian Rowing National Championships (2005)

- Silver medalist in the U-16 at the Russian Rowing National Championships (2004)

- Bronze medalist in the U-16 at National Rowing Championships (2003)

\section{Community Service:}

Louisville, $K Y$

Over a 100 hours of community service:

- Brain Days at the Kentucky Science center (2015-2017)

- $\quad$ Fit4Me (2008-2011)

- Festival of Trees and Lights (2006-2010)

- Habitat for Humanities (2009-2011)

- Project Warm $(2006,2007)$

- Ironman (2008) 